\title{
WestVirginiaUniversity
}

THE RESEARCH REPOSITORY @ WVU

Graduate Theses, Dissertations, and Problem Reports

2012

\section{Group Connectivity of Graphs}

Senmei Yao

West Virginia University

Follow this and additional works at: https://researchrepository.wvu.edu/etd

\section{Recommended Citation}

Yao, Senmei, "Group Connectivity of Graphs" (2012). Graduate Theses, Dissertations, and Problem Reports. 3567.

https://researchrepository.wvu.edu/etd/3567

This Dissertation is protected by copyright and/or related rights. It has been brought to you by the The Research Repository @ WVU with permission from the rights-holder(s). You are free to use this Dissertation in any way that is permitted by the copyright and related rights legislation that applies to your use. For other uses you must obtain permission from the rights-holder(s) directly, unless additional rights are indicated by a Creative Commons license in the record and/ or on the work itself. This Dissertation has been accepted for inclusion in WVU Graduate Theses, Dissertations, and Problem Reports collection by an authorized administrator of The Research Repository @ WVU.

For more information, please contact researchrepository@mail.wvu.edu. 


\title{
Group Connectivity of Graphs
}

\author{
Senmei Yao \\ Dissertation submitted to the \\ Eberly College of Arts and Sciences \\ at West Virginia University \\ in partial fulfillment of the requirements \\ for the degree of \\ Doctor of Philosophy \\ in \\ Mathematics \\ Hong-Jian Lai, Ph.D., Chair \\ K. Subramani, Ph.D. \\ John Goldwasser, Ph.D. \\ Jerzy Wojciechowski, Ph.D. \\ Cun-Quan Zhang, Ph.D. \\ Department of Mathematics \\ West Virginia University \\ Morgantown, WV, 26505 \\ 2012
}

Keywords: nowhere-zero flow, group connectivity, line graph 


\section{ABSTRACT \\ Group Connectivity of Graphs}

\section{Senmei Yao}

Tutte introduced the theory of nowhere-zero flows and showed that a plane graph $G$ has a face $k$-coloring if and only if $G$ has a nowhere-zero $A$-flow, for any Abelian group $A$ with $|A| \geq k$. In 1992 Jaeger et al [16] extended nowhere-zero flows to group connectivity of graphs: given an orientation $D$ of a graph $G$, if for any $b: V(G) \mapsto A$ with $\sum_{v \in V(G)} b(v)=0$, there always exists a map $f: E(G) \mapsto A-\{0\}$, such that at each $v \in V(G)$,

$$
\sum_{e=v w \text { is directed from } v \text { to } w} f(e)-\sum_{e=u v \text { is directed from } u \text { to } v} f(e)=b(v)
$$

in $A$, then $G$ is $A$-connected. For a 2-edge-connected graph $G$, define $\Lambda_{g}(G)=\min \{k$ : for any Abelian group $A$ with $|A| \geq k, G$ is $A$-connected\}.

Let $G_{1} \otimes G_{2}$ and $G_{1} \times G_{2}$ denote the strong and Cartesian product of two connected nontrivial graphs $G_{1}$ and $G_{2}$. We prove that $\Lambda_{g}\left(G_{1} \otimes G_{2}\right) \leq 4$, where equality holds if and only if both $G_{1}$ and $G_{2}$ are trees and $\min \left\{\left|V\left(G_{1}\right)\right|,\left|V\left(G_{2}\right)\right|\right\}=2 ; \Lambda_{g}\left(G_{1} \times G_{2}\right) \leq 5$, where equality holds if and only if both $G_{1}$ and $G_{2}$ are trees and either $G_{1} \cong K_{1, m}$ and $G_{2} \cong K_{1, n}$, for $n, m \geq 2$ or $\min \left\{\left|V\left(G_{1}\right)\right|,\left|V\left(G_{2}\right)\right|\right\}=2$. A similar result for the lexicographical product graphs is also obtained.

Let $P$ denote a path in $G$, let $\beta_{G}(P)$ be the minimum length of a circuit containing $P$, and let $\beta_{i}(G)$ be the maximum of $\beta_{G}(P)$ over paths of length $i$ in $G$. We show that $\Lambda_{g}(G) \leq \beta_{i}(G)+1$ for any integer $i>0$ and for any 2-connected graph $G$. Partial solutions toward determining the graphs for which equality holds were obtained by Fan et al. in [J. Comb. Theory, Ser. B, 98(6) (2008), 1325-1336], among others. We completely determine all graphs $G$ with $\Lambda_{g}(G)=$ $\beta_{2}(G)+1$.

Let $\mathbb{Z}_{3}$ denote the cyclic group of order 3 . In [16], Jaeger et al conjectured that every 5 -edgeconnected graph is $\mathbb{Z}_{3}$-connected. We proved the following:

(i) Every 5-edge-connected graph is $\mathbb{Z}_{3}$-connected if and only if every 5-edge-connected line graph is $\mathbb{Z}_{3}$-connected.

(ii) Every 6-edge-connected triangular line graph is $\mathbb{Z}_{3}$-connected.

(iii) Every 7-edge-connected triangular claw-free graph is $\mathbb{Z}_{3}$-connected.

In particular, every 6-edge-connected triangular line graph and every 7-edge-connected triangular claw-free graph have a nowhere-zero 3-flow. 


\section{Acknowledgements}

First and foremost, I am most indebted to my supervisor, Dr. Hong-Jian Lai, for his continued encouragement and support over these last few years. It is a pleasure to work under his supervision. Without him, this paper could not have come about.

I would also like to thank my other committee members: Dr. K. Subramani, Dr. John Goldwasser, Dr. Jerzy Wojciechowski, and Dr. Cun-Quan Zhang, for their help during my studies.

And finally, I would like to thank the Department of Mathematics and Eberly College of Arts and Sciences at West Virginia University for providing me with an excellent study environment and support during my study as a graduate student. 


\section{DEDICATION}

To

My Parents 


\section{Contents}

1 Preliminaries 1

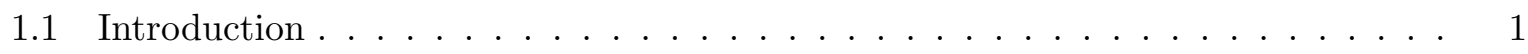

1.2 Notation and Terminology . . . . . . . . . . . . . . . . . . 3

1.3 Known Results . . . . . . . . . . . . . . . . . . . . . . . . . 4

1.4 Main Results . . . . . . . . . . . . . . . . . . . . 5 5

2 Group Connectivity in Products of Graphs $\quad 7$

2.1 Introduction and Main Results . . . . . . . . . . . . . . . . . . 7

2.2 Strong Products and Lexicographical Products . . . . . . . . . . . . . . . . 8

2.3 Cartesian Products . . . . . . . . . . . . . . . . . . . . 10

3 Group Connectivity in Line Graphs $\quad 17$

3.1 Introduction . . . . . . . . . . . . . . . . . . . . . 17

3.2 Ryjácěk ([32]) Closure of a Claw-free Graph . . . . . . . . . . . . . . . . 18

4 Group Connectivity of $J_{3}$ Line Graphs and $J_{3}$ claw-free Graphs 23

4.1 Introduction . . . . . . . . . . . . . . . . . . . . 23

4.2 Main Results . . . . . . . . . . . . . . . . . . . . . . . . 24

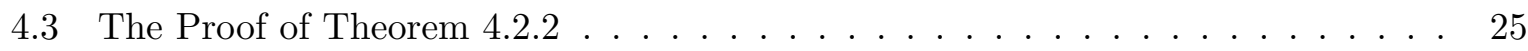

5 An Upper Bound on Group Connectivity 35

5.1 Introduction . . . . . . . . . . . . . . . . . . 35

5.2 Characterization of the Extremal Graphs . . . . . . . . . . . . . . . . 41

5.3 Applications . . . . . . . . . . . . . . . . . . 47 


\section{Chapter 1}

\section{Preliminaries}

\section{$1.1 \quad$ Introduction}

Undefined terms and notations will follow Bondy and Murty [2]. And $\kappa(G), \kappa^{\prime}(G)$, and $\delta(G)$ denote the connectivity, edge-connectivity, and minimum degree of a graph $G$, respectively. Different from [2], a 2-regular nontrivial connected graph is called a circuit, and a circuit with $k$ edges is referred as a $k$-circuit. A subgraph $H$ of $G$ is a clique if $H$ is isomorphic to a complete graph.For an edge subset $J \subseteq E(G)$ of a graph $G$, we take the convention of using $J$ to denote both the edge subset as well as the subgraph $G[J]$ induced by the edge set $J$. Throughout this paper $A$ denotes an (additive) Abelian group with identity 0. and $A^{*}=A-\{0\}$.

Let $D=D(G)$ be an orientation of a graph $G$. If an edge $e \in E(G)$ is directed from a vertex $u$ to a vertex $v$, then let $\operatorname{tail}(e)=u$ and $h e a d(e)=v$. For a vertex $v \in V(G)$, define

$$
E_{D}^{+}(v)=\{e \in E(G): v=\operatorname{tail}(e)\}, \text { and } E_{D}^{-}(v)=\{e \in E(G): v=\operatorname{head}(e)\}
$$

Following Jaeger et al [16], we define $F(G, A)=\{f \mid f: E(G) \mapsto A\}$ and $F^{*}(G, A)=\{f \mid f: E(G) \mapsto$ $\left.A^{*}\right\}$. For a function $f: E(G) \rightarrow A$, define $\partial f: V(G) \mapsto A$ by

$$
\partial f(v)=\sum_{e \in E_{D}^{+}(v)} f(e)-\sum_{e \in E_{D}^{-}(v)} f(e),
$$

where " $\sum$ " refers to the addition in $A$.

Assume that $G$ has an orientation $D(G)$. A function $b: V(G) \mapsto A$ is called an $A$-valued zero sum function on $G$ if $\sum_{v \in V(G)} b(v)=0$. The set of all $A$-valued zero sum functions on $G$ is denoted by $Z(G, A)$. A function $f \in F(G, A)$ is an $A$-flow of $G$ if $\partial f(v)=0$ for every vertex $v \in V(G)$. An $A$-flow $f$ is a nowhere-zero $A$-flow (abbreviated as $A$-NZF) if $f \in F^{*}(G, A)$. For a $b \in Z(G, A)$, a function $f \in F^{*}(G, A)$ is a nowhere-zero $(A, b)$-flow (abbreviated as $(A, b)-\mathrm{NZF})$ if $\partial f=b$. A graph $G$ is $A$-connected if for all $b \in Z(G, A), G$ has an $(A, b)$-NZF. 
Let $\langle A\rangle$ be the family of graphs that are $A$-connected. The group connectivity number of a graph $G$ is defined as

$$
\Lambda_{g}(G)=\min \{k: G \in\langle A\rangle \text { for every Abelian group } A \text { with }|A| \geq k\} .
$$

The concept of group connectivity was first introduced by Jaeger, Linial, Payan, and Tarsi in [16] as a nonhomogeneous form of the nowhere-zero flow problem. The nowhere-zero flow problem was first introduced by Tutte [36] in his way to attach the 4-color-conjecture. Tutte left with several fascinating conjectures in this area, which have remained open as of today.

Conjecture 1.1.1 (Tutte [36], [15])

(i) Every graph $G$ with $\kappa^{\prime}(G) \geq 2$ has a nowhere-zero $\mathbb{Z}_{5}$-flow.

(ii) Every graph $G$ with $\kappa^{\prime}(G) \geq 2$ without a subgraph contractible to the Peterson graph admits a nowhere-zero $\mathbb{Z}_{4}$-flow.

(iii) Every graph $G$ with $\kappa^{\prime}(G) \geq 4$ admits a nowhere-zero $\mathbb{Z}_{3}$-flow.

Jaeger et al made the following conjectures about group connectivity. The truth of these conjectures will imply the truth of Tutte's $\mathbb{Z}_{5}$-flow conjecture and $\mathbb{Z}_{3}$-flow conjecture, as indicated by Kochol [17].

Conjecture 1.1.2 (Jaeger, Linial, Payan and Tarsi [16])

(i) If $G$ is a 3-edge-connected graph, then $\Lambda_{g}(G) \leq 5$.

(ii) If $G$ is a 5-edge-connected graph, then $\Lambda_{g}(G) \leq 3$.

(iii) There exists an integer $k \geq 5$ such that if $\kappa^{\prime}(G) \geq k$, then $\Lambda_{g}(G) \leq 3$

While many have contributed to the literature of nowhere-zero flows, all these conjectures remain open. Several researchers have investigated the problem what kind of products graphs will have nowhere-zero $A$-flows when $|A|$ is small, as seen in [13], and [34] and [40].

In [38], Xu and Zhang proposed a triangulated version of the 3 -flow conjecture. Let $J_{3}$ denote the family of all connected graphs such that $G \in J_{3}$ if and only if every edge of $G$ lies in a $K_{3}$ of $G$. A graph in $J_{3}$ will also be referred as a $J_{3}$ graph.

Conjecture 1.1.3 (Xu and Zhang, [38]) If $\kappa^{\prime}(G) \geq 4$ and if $G \in J_{3}$, then $G$ has a 3-NZF.

Devos (Problem 1 in [28]) suggested that if $\kappa^{\prime}(G) \geq 4$ and if $G \in J_{3}$, then $\Lambda_{g}(G) \leq 3$. But a counterexample to this stronger version was given in [28], where a modified version of the conjecture is proposed: If $\kappa^{\prime}(G) \geq 5$ and if $G \in J_{3}$, then $G$ has a 3-NZF.

There have been lots of researches conducted to attack Conjectures 1.1.1 and 1.1.2. See Jaeger [15] and Zhang [39] for literature surveys. Jeager [14] was the first to show that every 2edge-connected graph has an 8-NZF, and that every 4-edge-connected graph has a 4-NZF. Later 
Seymour [33] proved that every 2-edge-connected graph has a 6-NZF. Jaeger, Linial, Payan and Tarsi [16] further showed that if $G$ is a 3-edge-connected graph, then $\Lambda_{g}(G) \leq 6$. More recently, Sudakov [35] showed that almost every random graph with minimum degree at least 2 has a 3-NZF. As for highly connected graphs, Lai and Zhang [19] first proved that every $4 \log _{2}|V(G)|$-edge-connected graph has a 3-NZF. More recently in [26], it is proved that every $3 \log _{2}|V(G)|$-edge-connected graph is $\mathbb{Z}_{3}$-connected.

\subsection{Notation and Terminology}

For $m, n \geq 1, P_{m}$ is a path with $m$ edges, $K_{m}$ is a complete graph with $m$ vertices, and $K_{m, n}$ is a complete bipartite graph with bipartition $(X, Y)$ such that $|X|=m$ and $|Y|=n$.

Let $H_{1}$ and $H_{2}$ be two subgraphs of a graph $G$. We say that $G$ is a parallel connection of $H_{1}$ and $H_{2}$, denoted by $H_{1} \oplus_{2} H_{2}$, if $E\left(H_{1}\right) \cup E\left(H_{2}\right)=E(G),\left|V\left(H_{1}\right) \cap V\left(H_{2}\right)\right|=2$ and $\left|E\left(H_{1}\right) \cap E\left(H_{2}\right)\right|=1$. The edge $e \in E\left(H_{1}\right) \cap E\left(H_{2}\right)$ is usually referred as the base edge.

A wheel $W_{k}$ is the graph obtained from a $k$-circuit by adding a new vertex the center of the wheel, and then by joining the center to every vertex of the $k$-circuit. A fan $F_{k}$ is the graph obtained from $W_{k}$ by deleting an edge not incident with the center. Note that $F_{2}$ is the 3 -circuit, and $W_{3}$ is the complete graph $K_{4}$. The family $\langle W F\rangle$ can now be recursively defined as follows:

(WF1) For all $k \geq 1$, and $n \geq 2, W_{2 k+1}, F_{n} \in\langle W F\rangle$.

(WF2) If $G, H \in\langle W F\rangle$, then any parallel connection of $G$ and $H$ is also in $\langle W F\rangle$.

Graphs in $\langle W F\rangle$ are usually referred as $W F$-graphs. For an integer $k \geq 3$, graph $G$ is $k$-circuit connected if for any pair of edges $e, e^{\prime} \in E(G), G$ has a sequence of circuits $C_{1}, C_{2}, \cdots, C_{m}$ such that $\left|E\left(C_{i}\right)\right| \leq k,(1 \leq i \leq m), e \in E\left(C_{1}\right), e^{\prime} \in E\left(C_{m}\right)$ and $E\left(C_{i}\right) \cap$ $E\left(C_{i+1}\right) \neq \varnothing,(1 \leq i \leq m-1)$. The sequence $C_{1}, C_{2}, \cdots, C_{m}$ is often referred as an $\left(e, e^{\prime}\right)-k$ circuit-path. A 3-circuit connected graph is also referred as a triangularly connected graph. By definition, every $W F$-graph is triangularly connected.

A graph $G$ is collapsible if for every even subset $R \subseteq V(G), G$ has a subgraph $\Gamma_{R}$ (called the $R$-subgraph of $G$ ) such that $G-E\left(\Gamma_{R}\right)$ is connected and $R$ is the set of odd-degree vertices of $\Gamma_{R}$. The collection of all collapsible graphs is denoted by $\mathcal{C L}$. The following summarizes some useful result related to collapsible graphs.

A $J_{3}$ graph $G$ is triangularly connected if for all $e, e^{\prime} \in E(G), G$ has a sequence of circuits $C^{1}, C^{2}, \ldots, C^{m}$ in $G$ such that each of the following holds.

(TC1) $e \in E\left(C^{1}\right)$ and $e^{\prime} \in E\left(C^{m}\right)$,

(TC2) for all $1 \leq i \leq m,\left|E\left(C^{i}\right)\right| \leq 3$, and

(TC3) for all $1 \leq i \leq m-1,\left|E\left(C^{i}\right) \cap E\left(C^{i+1}\right)\right|>0$.

The sequence $\left\{C^{1}, C^{2}, \cdots, C^{m}\right\}$ will be referred as an $\left(e, e^{\prime}\right)$-triangle-path in $G$. Graphs in 
$\langle W F\rangle$ are usually referred as $W F$-graphs. By definition, every $W F$-graph is triangularly connected.

We follow the notations in [18]. Let $G$ be a graph with $C_{4}$ as a subgraph, and $\pi=\{X, Y\}$ the bipartition of $V\left(C_{4}\right)$ so that both $X$ and $Y$ are independent sets of $C_{4}$. Let $G / \pi$ denote the graph obtained from $G$ by identifying all vertices of $X$ to form a single vertex $x$, identifying all vertices of $Y$ to form a single vertex $y$, and then joining $x, y$ with a new edge $e_{\pi}=x y$, so that

$$
E(G)-E\left(C_{4}\right)=E(G / \pi)-\left\{e_{\pi}\right\}
$$

Let $P$ denote a path in $G$, let $\beta_{G}(P)$ be the minimum length of a circuit containing $P$, and let $\beta_{i}(G)$ be the maximum of $\beta_{G}(P)$ over paths of length $i$ in $G$.

\subsection{Known Results}

Proposition 1.3.1 (Proposition 3.2 of [21]) Let $A$ be an Abelian group with $|A| \geq 3$. Then $\langle A\rangle$ satisfies each of the following:

(C1) $K_{1} \in\langle A\rangle$,

(C2) if $G \in\langle A\rangle$ and if $e \in E(G)$, then $G / e \in\langle A\rangle$,

(C3) if $H$ is a subgraph of $G$ and if both $H \in\langle A\rangle$ and $G / H \in\langle A\rangle$, then $G \in\langle A\rangle$.

Theorem 1.3.2 (Proposition 2.2 of [16]) Let $G$ be a connected graph and $A$ be an Abelian group. The following are equivalent.

(i) $G \in\langle A\rangle$.

(ii) For all $\bar{f} \in F(G, A)$, there exists $f \in F_{0}(G, A)$ such that for all $e \in E(G), f(e) \neq \bar{f}(e)$.

(iii) For all $b \in Z(G, A)$, and for all $\bar{f} \in F(G, A)$, there exists $f \in F(G, A)$ such that $\partial f=b$ and for all $e \in E(G), f(e) \neq \bar{f}(e)$.

Lemma 1.3.3 Let $G$ be a graph and $A$ be an Abelian group with $|A| \geq 3, K_{n}$ a complete graph of order $n$, and let $C_{n}$ denote the circuit on $n$ vertices (also referred as an n-circuit).

(i) (Lemma 2.1 of [23]) If for every edge e in a spanning tree of $G, G$ has a subgraph $H_{e} \in\langle A\rangle$ with $e \in E\left(H_{e}\right)$, then $G \in\langle A\rangle$.

(ii) ([16] and Lemma 3.3 of [21]) $\Lambda_{g}\left(C_{n}\right)=n+1$.

(iii) (Lemma 2.8 of [5], Lemma 2.6 of [9]) For any integer $k>1, \Lambda_{g}\left(W_{2 k}\right)=3$.

(iv) (Corollary 3.5 of [21]) Let $n \geq 5$ be an integer. Then $K_{n} \in\langle A\rangle$.

Theorem 1.3.4 Let $G$ be a graph and $H$ be a collapsible subgraph of $G$. Each of the following holds.

(i) (Catlin, Theorem 3 and its Corollary of [4]) $G$ is collapsible if and only if $G / H$ is collapsible.

(ii) (Catlin, Lemma 3 of [4]) If $G$ is collapsible, then for any $e \in E(G), G / e$ is collapsible. 
(iii) (Catlin, [4] and Lemma 1 of [3]) Let $e \in E\left(K_{3,3}\right)$. Then $C_{2}, C_{3}, K_{3,3}$ and $K_{3,3}-e$ are collapsible.

(iv) (Theorem 1.5, [20]) Let $A$ be an Abelian group with $|A|=4$. Then $H \in\langle A\rangle$.

Theorem 1.3.5 (Fan et al, [9]) Let $G$ be a triangularly connected graph with $|V(G)| \geq 3$. Then

(i) $G$ is $\mathbb{Z}_{3}$-connected if and only if $G$ contains a nontrivial $\mathbb{Z}_{3}$-connected subgraph.

(ii) $G$ is $\mathbb{Z}_{3}$-connected if and only if $G \notin\langle W F\rangle$.

Theorem 1.3.6 (Fan et al, [9]) Let $G$ be a triangularly connected graph with $|V(G)| \geq 2$. Each of the following holds.

(i) (Theorem 1.4 of [9]) $G$ is $\mathbb{Z}_{3}$-connected if and only if $G \notin\langle W F\rangle$.

(ii) (Lemma 2.4 of [9]) $G$ is $\mathbb{Z}_{3}$-connected if and only if $G$ contains a nontrivial $\mathbb{Z}_{3}$-connected subgraph.

The following is an immediate corollary of Theorem 1.3.6 and Lemma 1.3.3(ii) and (iii).

Corollary 1.3.7 If $G \in\langle W F\rangle$, then $G$ does not contain any even wheel or 2-circuit.

Lemma 1.3.8 Let $G$ be a connected graph, and let $A$ be an Abelian group.

(i) (Lemma 2.1 of [23]) Let $T$ be a connected spanning subgraph of $G$. If for each edge e $\in E(T)$, $G$ has a subgraph $H_{e} \in\langle A\rangle$ with $e \in E\left(H_{e}\right)$, then $G \in\langle A\rangle$.

(ii) $G \in\langle A\rangle$ if and only if every block of $G$ is A-connected.

\subsection{Main Results}

The main results in the dissertation is as following.

1. (i) $\Lambda_{g}\left(G_{1} \otimes G_{2}\right) \leq 4$, where equality holds if and only if both $G_{1}$ and $G_{2}$ are trees and $\min \left\{\left|V\left(G_{1}\right)\right|,\left|V\left(G_{2}\right)\right|\right\}=2$.

(ii) $\Lambda_{g}\left(G_{1}\left[G_{2}\right]\right) \leq 4$, where equality holds if and only if both $G_{1}$ and $G_{2}$ are trees and $\min \left\{\left|V\left(G_{1}\right)\right|,\left|V\left(G_{2}\right)\right|\right\}=2$.

(iii) $\Lambda_{g}\left(G_{1} \times G_{2}\right) \leq 5$, where equality holds if and only if either $G_{1} \cong K_{1, m}$ and $G_{2} \cong K_{1, n}$, for $n, m \geq 2$ or $G_{1}$ is a tree and $G_{2} \cong K_{2}$.

2.(i) Every 5 -edge-connected graph is $\mathbb{Z}_{3}$-connected if and only if every 5 -edge-connected line graph is $\mathbb{Z}_{3}$-connected.

(ii) Every 6-edge-connected triangular line graph is $\mathbb{Z}_{3}$-connected.

(iii) Every 7-edge-connected triangular claw-free graph is $\mathbb{Z}_{3}$-connected.

(iv) In particular, every 6-edge-connected triangular line graph has a nowhere-zero 3-flow, and every 7 -edge-connected triangular claw-free graph has a nowhere-zero 3 -flow. 
3. $\Lambda_{g}(G) \leq \beta_{i}(G)+1$ for any integer $i>0$ and for any 2-connected graph $G$. Partial solutions toward determining the graphs for which equality holds were obtained by Fan et al. in [J. Comb. Theory, Ser. B, 98(6) (2008), 1325-1336], among others. In this paper, we completely determine all graphs $G$ with $\Lambda_{g}(G)=\beta_{2}(G)+1$. 


\section{Chapter 2}

\section{Group Connectivity in Products of Graphs}

\subsection{Introduction and Main Results}

We consider finite graphs which may have multiple edges but no loops.

For graph products, we adopt the notation in [10]. Let $G_{1}, G_{2}$ be two graphs. The Cartesian product graph $G=G_{1} \times G_{2}$ is a graph with vertex set $V(G)=V\left(G_{1}\right) \times V\left(G_{2}\right)$ and edge set $E(G)=\left\{\left(u_{1}, u_{2}\right)\left(v_{1}, v_{2}\right) \mid u_{1}=v_{1}\right.$ and $u_{2} v_{2} \in E\left(G_{2}\right)$ or $u_{2}=v_{2}$ and $\left.u_{1} v_{1} \in E\left(G_{1}\right)\right\}$. The strong product graph $G=G_{1} \otimes G_{2}$ is a graph with vertex set $V(G)=V\left(G_{1}\right) \times V\left(G_{2}\right)$ and edge set $E(G)=\left\{\left(u_{1}, u_{2}\right)\left(v_{1}, v_{2}\right) \mid u_{1}=v_{1}\right.$ and $u_{2} v_{2} \in E\left(G_{2}\right)$, or $u_{2}=v_{2}$ and $u_{1} v_{1} \in E\left(G_{1}\right)$, or both $u_{1} v_{1} \in E\left(G_{1}\right)$ and $\left.u_{2} v_{2} \in E\left(G_{2}\right)\right\}$. And the lexicographic product (sometimes called composition, tensor or wreath product) $G=G_{1}\left[G_{2}\right]$ is a graph with vertex set $V(G)=V\left(G_{1}\right) \times$ $V\left(G_{2}\right)$ and edge set $E(G)=\left\{\left(u_{1}, u_{2}\right)\left(v_{1}, v_{2}\right) \mid u_{1} v_{1} \in E\left(G_{1}\right)\right.$, or $u_{1}=v_{1}$ and $\left.u_{2} v_{2} \in E\left(G_{2}\right)\right\}$.

The purpose of this chapter is to determine the group connectivity for all strong product and lexicographical product graphs, and to investigate the group connectivity number of the Cartesian product graphs.

The following are immediate from the above definitions.

Proposition 2.1.1 Each of the following holds.

(i) $G_{1} \times G_{2}$ is a spanning subgraph of $G_{1} \otimes G_{2}$, and $G_{1} \otimes G_{2}$ is a spanning subgraph of $G_{1}\left[G_{2}\right]$.

(ii) If $G_{2} \cong K_{m}$ is a complete graph, then $G_{1}\left[K_{m}\right]=G_{1} \otimes K_{m}$.

In this paper we will determine the group connectivity number of certain products of connected graphs by proving the following main results.

Theorem 2.1.2 $\Lambda_{g}\left(G_{1} \otimes G_{2}\right) \leq 4$, where equality holds if and only if both $G_{1}$ and $G_{2}$ are trees and $\min \left\{\left|V\left(G_{1}\right)\right|,\left|V\left(G_{2}\right)\right|\right\}=2$. 
Corollary 2.1.3 $G_{1} \otimes G_{2}$ has a nowhere-zero 3-flow if and only if either one of $G_{1}$ and $G_{2}$ is not a tree, or both $G_{1}$ and $G_{2}$ are trees with $\min \left\{\left|V\left(G_{1}\right)\right|,\left|V\left(G_{2}\right)\right|\right\} \geq 3$.

Theorem 2.1.4 $\Lambda_{g}\left(G_{1}\left[G_{2}\right]\right) \leq 4$, where equality holds if and only if both $G_{1}$ and $G_{2}$ are trees and $\min \left\{\left|V\left(G_{1}\right)\right|,\left|V\left(G_{2}\right)\right|\right\}=2$.

Corollary 2.1.5 $G_{1}\left[G_{2}\right]$ has a nowhere-zero 3-flow if and only if either one of $G_{1}$ and $G_{2}$ is not a tree, or both $G_{1}$ and $G_{2}$ are trees with $\min \left\{\left|V\left(G_{1}\right)\right|,\left|V\left(G_{2}\right)\right|\right\} \geq 3$.

Theorem 2.1.6 $\Lambda_{g}\left(G_{1} \times G_{2}\right) \leq 5$, where equality holds if and only if either $G_{1} \cong K_{1, m}$ and $G_{2} \cong K_{1, n}$, for $n, m \geq 2$ or $G_{1}$ is a tree and $G_{2} \cong K_{2}$.

\subsection{Strong Products and Lexicographical Products}

The following observation follows from the definition of strong product immediately.

$$
G_{1} \otimes G_{2} \text { is triangularly connected. }
$$

Thus every edge of $G_{1} \otimes G_{2}$ is contained in a 3-circuit. It follows by Lemma 1.3.6 and Proposition 1.3.7, that

$$
\Lambda_{g}\left(G_{1} \otimes G_{2}\right) \leq 4 .
$$

We shall prove Theorem 2.1.2 by proving each of the following lemmas.

Lemma 2.2.1 Each of the following holds.

(i) $\Lambda_{g}\left(C_{n} \otimes K_{2}\right)=3$ and $\Lambda_{g}\left(P_{2} \otimes P_{2}\right)=3$.

(ii) If $G_{1}$ or $G_{2}$ contains a circuit, then $\Lambda_{g}\left(G_{1} \otimes G_{2}\right)=3$.

(iii) If both $G_{1}$ and $G_{2}$ contains a path of length at least 2, then $\Lambda_{g}\left(G_{1} \otimes G_{2}\right)=3$.

Proof. (i) By (2.2), $\Lambda_{g}\left(C_{n} \otimes K_{2}\right) \leq 4$. By the definition of strong product, $C_{n} \otimes K_{2}$ is not a $W F$-graph. It follows by $(2.1)$ and by Theorem 1.3 .5 that $C_{n} \otimes K_{2}$ is $\mathbb{Z}_{3}$-connected. Thus $\Lambda_{g}\left(C_{n} \otimes K_{2}\right)=3$.

Let $G=P_{2} \otimes P_{2}$ (see Figure 2.1). Then the subgraph $G^{\prime}$ induced by the vertex subset $\left\{x_{0} y_{1}, x_{1} y_{0}, x_{1} y_{1}, x_{1} y_{2}, x_{2} y_{1}\right\}$ is an even wheel. By Theorem 1.3.5(ii), $\Lambda_{g}\left(G^{\prime}\right)=3$. And by Theorem 1.3.5(i), $\Lambda_{g}(G)=3$.

(ii) and (iii) Both conclusions follow from (i) and from Theorem 1.3.5(i).

Lemma 2.2.2 If $G_{1}$ is a tree, and $G_{2} \cong K_{2}$, then each of the following holds.

(i) $G_{1} \otimes G_{2} \in\langle W F\rangle$.

(ii) $\Lambda_{g}\left(G_{1} \otimes G_{2}\right)=4$. 


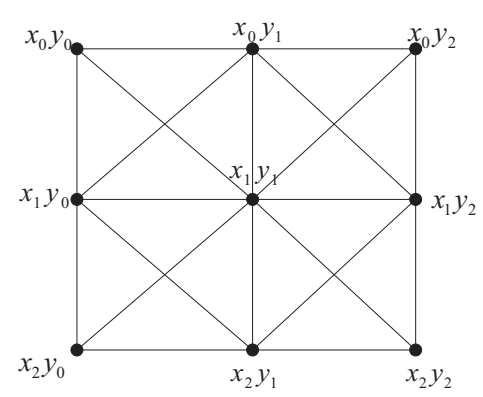

Figure 2.1 $G=P_{2} \otimes P_{2}$

(iii) If $H$ is a nontrivial connected graph, then $H$ has a nowhere-zero $\mathbb{Z}_{3}$-flow if and only if $H \oplus_{2} K_{4}$ has a nowhere-zero $\mathbb{Z}_{3}$-flow.

(iv) $G_{1} \otimes G_{2}$ does not have a nowhere-zero $\mathbb{Z}_{3}$-flow.

Proof. (i) We first argue by induction on $\left|V\left(G_{1}\right)\right|$ to show that, under the assumption of this lemma, $G_{1} \otimes G_{2} \in\langle W F\rangle$. If $\left|V\left(G_{1}\right)\right|=2$, then $G_{1} \cong K_{2}$ as well, and so $G_{1} \otimes G_{2} \cong K_{4} \in\langle W F\rangle$. Assume that for smaller values of $\left|V\left(G_{1}\right)\right|$, if $G_{1}$ is a tree, then $G_{1} \otimes G_{2} \in\langle W F\rangle$. Assume now $\left|V\left(G_{1}\right)\right| \geq 3$. Since $G_{1}$ is a tree, $G_{1}$ has an edge $u v$ such that $u$ has degree 1 in $G_{1}$. It follows by assumption that $\left(G_{1}-u\right) \otimes G_{2} \in\langle W F\rangle$. By the definition of strong product, $G_{1} \otimes G_{2}$ is a parallel connection of $\left(G_{1}-u\right) \otimes G_{2}$ and $G_{1}[\{u, v\}] \otimes G_{2} \cong K_{4}$. It follows by (WF2) in the definition of $\langle W F\rangle$ that $G_{1} \otimes G_{2} \in\langle W F\rangle$. Hence (i) holds by induction.

(ii) By (2.2), (i) and Theorem 1.3.5, (ii) follows as well.

(iii) Suppose that the $K_{4}$, as a subgraph of $H \oplus_{2} K_{4}$, has four vertices $u_{1}, v_{1}, u_{2}, v_{2}$ such that $u_{1}, v_{1}$ are the two vertices not in $V(H)$. If $H$ has a nowhere-zero 3 -flow, then extend the orientation of $H$ to $H \oplus_{2} K_{4}$ by orienting all edges incident with $u_{1}$ away from $u_{1}$ and all edges incident with $v_{1}$ into $v_{1}$, and by extending the flow ion $H$ to $E\left(K_{4}\right)-E(H)$ taking a constant value 1 . Then we obtain a nowhere-zero $\mathbb{Z}_{3}$-flow of $H \oplus_{2} K_{4}$. Conversely, if $H_{\oplus_{2}} K_{4}$ has a nowhere-zero $\mathbb{Z}_{3}$-flow, then since both $u_{1}$ and $v_{1}$ are adjacent degree 3 vertices, the restriction of this $\mathbb{Z}_{3}$-flow to $E(H)$ is also a nowhere-zero $\mathbb{Z}_{3}$-flow of $L$.

(iv) This follows from (iii) and by induction on $\left|V\left(G_{1}\right)\right|$.

Proof of Theorem 2.1.2: By (2.2) and by Lemma 2.2.2, we may assume that $\Lambda_{g}\left(G_{1} \otimes G_{2}\right)=4$ to prove that both $G_{1}$ and $G_{2}$ are trees, and $\min \left\{\left|V\left(G_{1}\right)\right|,\left|V\left(G_{2}\right)\right|\right\}=2$.

If $G_{1}$ or $G_{2}$ has a circuit, then by Lemma 2.2.1(ii), $G_{1} \otimes G_{2} \in\left\langle\mathbb{Z}_{3}\right\rangle$. Hence we may assume that both $G_{1}$ and $G_{2}$ are trees. If $\min \left\{\left|V\left(G_{1}\right)\right|,\left|V\left(G_{2}\right)\right|\right\} \geq 3$, then since $G_{1}$ and $G_{2}$ are connected, each of $G_{1}$ and $G_{2}$ contains a path of length 2. It follows by Lemma 2.2.1(i) that $G_{1} \otimes G_{2}$ 
has a nontrivial subgraph in $\left\langle\mathbb{Z}_{3}\right\rangle$, and so by (2.1) and by Theorem 1.3.5(i), $G_{1} \otimes G_{2} \in\left\langle\mathbb{Z}_{3}\right\rangle$. Therefore, we must have $\min \left\{\left|V\left(G_{1}\right)\right|,\left|V\left(G_{2}\right)\right|\right\}=2$. $\square$ Proof of Corollary 2.1.3: Since $G \in\left\langle\mathbb{Z}_{3}\right\rangle$ implies that $G$ has a nowhere-zero 3-flow, the sufficiency follows from Theorem 2.1.2. Conversely, if both $G_{1}$ and $G_{2}$ are trees, and $\min \left\{\left|V\left(G_{1}\right)\right|\right.$, $\left.\left|V\left(G_{2}\right)\right|\right\}=2$, then by Lemma 2.2.2(iv), $G_{1} \otimes G_{2}$ does not have a nowhere-zero $\mathbb{Z}_{3}$-flow.

Proof of Theorem 2.1.4: By Proposition 2.1.1(i), $G_{1} \otimes G_{2}$ is a spanning subgraph of $G_{1}\left[G_{2}\right]$. By Theorem 2.1.2 and Lemma 1.3.6, $\Lambda_{g}\left(G_{1}\left[G_{2}\right]\right) \leq 4$. If $G_{1} \otimes G_{2} \in\left\langle\mathbb{Z}_{3}\right\rangle$, then by Lemma 1.3.6, $G_{1}\left[G_{2}\right] \in\left\langle\mathbb{Z}_{3}\right\rangle$ as well. If both $G_{1}$ and $G_{2}$ are trees, and $\min \left\{\left|V\left(G_{1}\right)\right|,\left|V\left(G_{2}\right)\right|\right\}=2$, then by Proposition 2.1.1(ii), $G_{1}\left[G_{2}\right]=G_{1} \otimes G_{2}$, and so by Theorem 2.1.2, $G_{1}\left[G_{2}\right] \notin\left\langle\mathbb{Z}_{3}\right\rangle$.

Proof of Corollary 2.1.5: The proof is similar to that for Corollary 2.1.3, and so it is omitted.

\subsection{Cartesian Products}

For graph products, we adopt the notation in [10]. Let $G_{1}, G_{2}$ be two graphs. The Cartesian product graph $G=G_{1} \times G_{2}$ is a graph with vertex set $V(G)=V\left(G_{1}\right) \times V\left(G_{2}\right)$ and edge set $E(G)=\left\{\left(u_{1}, u_{2}\right)\left(v_{1}, v_{2}\right) \mid u_{1}=v_{1}\right.$ and $u_{2} v_{2} \in E\left(G_{2}\right)$ or $u_{2}=v_{2}$ and $\left.u_{1} v_{1} \in E\left(G_{1}\right)\right\}$.

Then the following observation follows from the definition of Cartesian product immediately.

$$
G_{1} \times G_{2} \text { is } 4 \text {-circuit connected. }
$$

Thus every edge of $G_{1} \times G_{2}$ is contained in a 4-circuit. It follows by Lemma 1.3.6 and Proposition 1.3.7 that

$$
\Lambda_{g}\left(G_{1} \times G_{2}\right) \leq 5
$$

Lemma 2.3.1 Let $G$ be 4-circuit connected and $A$ be an Abelian group with $|A|=4$. Each of the following holds.

(i) $G \in\langle A\rangle$ if and only if $G$ has a nontrivial A-connected subgraph.

(ii) $G \in \mathcal{C L}$ if and only if $G$ has a nontrivial collapsible subgraph.

(iii)If $G$ has a nontrivial A-connected subgraph, then $\Lambda_{g}(G) \leq 4$; If $G$ has a nontrivial collapsible subgraph, then $G$ is collapsible and $\Lambda_{g}(G) \leq 4$.

Proof. (i) If $G \in\langle A\rangle$, then $G$ is a nontrivial $A$-connected subgraph of $G$.

Conversely, let $H$ be a nontrivial maximal $A$-connected subgraph of $G$. If $G=H$, then done. Assume that $H \neq G$. Since $|E(H)| \geq 1$, there is an edge $e_{1} \in E(H)$. Since $E(G)-E(H) \neq \varnothing$, there is an edge $e_{2} \in E(G)-E(H)$. By the definition of 4-circuit-connectedness, $G$ has an $\left(e_{1}, e_{2}\right)$-4-circuit-path. By the choice of $e_{1}$ and $e_{2}$, this 4-circuit-path has a circuit $T$ with $|E(T)| \leq 4$ such that $T_{1}=E(T) \cap E(H) \neq \varnothing$ and $T_{2}=E(T)-T_{1} \neq \varnothing$. By Proposition 1.3.7, 


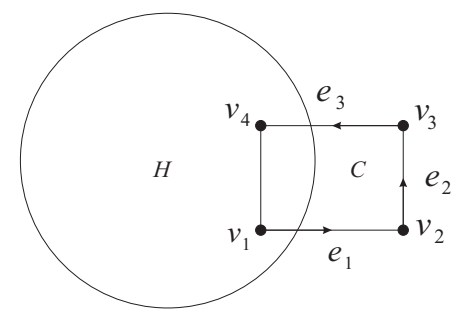

$G$

Figure $2.2 \mathrm{G}=\mathrm{H} \oplus_{2} \mathrm{C}$

$T / T_{1}$ is $A$-connected. Let $H^{\prime}=H \cup T$. Since $H^{\prime} / H=T / T_{1}$ is $A$-connected, and since $H$ is $A$-connected, it follows by Proposition 1.3.3(C3) that $H^{\prime}$ is $A$-connected, contrary to the maximality of $H$. Thus we must have $H=G$, and so $G$ is $A$-connected. This, together with ( 2.4), implies that $\Lambda_{g}(G) \leq 4$.

(ii) If $G \in\langle A\rangle$, then $G$ is a nontrivial collapsible subgraph of $G$.

Conversely, let $H$ be a nontrivial maximal collapsible subgraph of $G$. If $G=H$, then done. Assume that $H \neq G$. Since $|E(H)| \geq 1$, there is an edge $e_{1} \in E(H)$. Since $E(G)-E(H) \neq \varnothing$, there is an edge $e_{2} \in E(G)-E(H)$. By the definition of 4-circuit-connectedness, $G$ has an $\left(e_{1}, e_{2}\right)$ 4-circuit-path. By the choice of $e_{1}$ and $e_{2}$, this 4-circuit-path has a circuit $T$ with $|E(T)| \leq 4$ such that $T_{1}=E(T) \cap E(H) \neq \varnothing$ and $T_{2}=E(T)-T_{1} \neq \varnothing$. By Theorem 3.1.2(ii), $T / T_{1}$ is collapsible. Let $H^{\prime}=H \cup T$. Since $H^{\prime} / H=T / T_{1}$ is collapsible, and since $H$ is collapsible, it follows by Theorem 3.1.2(i) that $H^{\prime}$ is collapsible, contrary to the maximality of $H$. Thus we must have $H=G$, and so $G$ is collapsible.

(iii) This follows from (ii), ( 2.4) and Theorem 3.1.2(iv).

Lemma 2.3.2 Let $C$ be a 4-circuit and $A$ be an Abelian group with $|A|=4$. Let $G=H \oplus_{2} C$. Then $H$ is A-connected if and only if $G$ is A-connected.

Proof. Let $V(C)=\left\{v_{1}, v_{2}, v_{3}, v_{4}\right\}, E(C)=\left\{e_{1}, e_{2}, e_{3}, e_{4}\right\}$ and assume $V(H) \cap V(C)=\left\{v_{1}, v_{4}\right\}$ (see Figure 2.2). Let $D$ be an orientation of $G$ such that the edge $v_{i} v_{i+1}$ is directed from $v_{i}$ from $v_{i+1}$, for $i=1,2,3$.

If $H \in\langle A\rangle$, since $G / H$ is a 3-circuit, by Proposition 1.3.7, $G / H \in\langle A\rangle$, then, by Proposition 1.3.3 (C3), $G \in\langle A\rangle$.

Conversely, if $G \in A$, since for any Abelian group $A$ of order 4 , either $A \cong \mathbb{Z}_{4}$ or $A \cong Z_{2} \times Z_{2}$, then we have the following two cases.

Case 1: $A \cong \mathbb{Z}_{4}$. Let $b \in Z\left(H, \mathbb{Z}_{4}\right)$. Define $b^{\prime}: V(G) \mapsto \mathbb{Z}_{4}$ to be 


$$
b^{\prime}(v)= \begin{cases}1, & \text { if } v=v_{2}, v_{3} \\ b(v)+1, & \text { if } v=v_{1}, v_{4} \\ b(v), & \text { otherwise. }\end{cases}
$$

Then $\sum_{v \in V(G)} b^{\prime}(v)=\sum_{v \in V(H)} b(v)+4 \equiv 0(\bmod 4)$. So $b^{\prime} \in Z\left(G, \mathbb{Z}_{4}\right)$. By the definition of $\mathbb{Z}_{4}$-connectedness, there is a $\mathbb{Z}_{4}$-NZF $f^{\prime}$ of $G$ such that $\partial f^{\prime}(v)=b^{\prime}(v)$, for any $v \in V(G)$. So $f^{\prime}\left(e_{3}\right)=f^{\prime}\left(e_{2}\right)+1=f^{\prime}\left(e_{1}\right)+2$. Therefore $\left\{f^{\prime}\left(e_{3}\right), f^{\prime}\left(e_{2}\right), f^{\prime}\left(e_{1}\right)\right\}=\{1,2,3\}$. This concludes that $f^{\prime}\left(e_{1}\right)=1, f^{\prime}\left(e_{2}\right)=2$ and $f^{\prime}\left(e_{3}\right)=3$. Define $f: E(H) \mapsto \mathbb{Z}_{4}^{*}$ to be $f(e)=f^{\prime}(e)$, for any $e \in E(H)$. Then

$$
\partial f(v)= \begin{cases}\partial f^{\prime}(v)-1=b^{\prime}(v)-1=b(v), & \text { if } v=v_{1}, \\ \partial f^{\prime}(v)+3=b^{\prime}(v)+3=b(v)+4 \equiv b(v), & \text { if } v=v_{4}, \\ b(v), & \text { otherwise. }\end{cases}
$$

That is, for any $v \in V(H), \partial f(v)=b(v)$. Therefore by the definition of $\mathbb{Z}_{4}$-connectedness, $H \in\left\langle\mathbb{Z}_{4}\right\rangle$.

Case 2: $A \cong \mathbb{Z}_{2} \times \mathbb{Z}_{2}$.

Let $b \in Z\left(H, \mathbb{Z}_{2} \times \mathbb{Z}_{2}\right)$. Define $b^{\prime}: V(G) \mapsto \mathbb{Z}_{2} \times \mathbb{Z}_{2}$ to be

$$
b^{\prime}(v)= \begin{cases}b(v)+(0,1), & \text { if } v=v_{1}, \\ (1,0), & \text { if } v=v_{2}, \\ (0,1), & \text { if } v=v_{3} \\ b(v)-(1,0), & \text { if } v=v_{4} \\ b(v), & \text { otherwise }\end{cases}
$$

Then $\sum_{v \in V(G)} b^{\prime}(v)=\sum_{v \in V(H)} b(v)+2(0,1)=0$. So $b^{\prime} \in Z\left(G, \mathbb{Z}_{4}\right)$. By the definition of $\mathbb{Z}_{2} \times \mathbb{Z}_{2}$-connectedness, there is a $\mathbb{Z}_{2} \times \mathbb{Z}_{2}$-NZF $f^{\prime}$ of $G$ such that $\partial f^{\prime}(v)=b^{\prime}(v)$, for any $v \in V(G)$. So $f^{\prime}\left(e_{3}\right)=f^{\prime}\left(e_{2}\right)+(0,1)=f^{\prime}\left(e_{1}\right)+(1,1)$. Therefore $\left\{f^{\prime}\left(e_{3}\right), f^{\prime}\left(e_{2}\right), f^{\prime}\left(e_{1}\right)\right\}=$ $\{(0,1),(1,0),(1,1)\}$. This concludes that $f^{\prime}\left(e_{1}\right)=(0,1), f^{\prime}\left(e_{2}\right)=(1,1)$ and $f^{\prime}\left(e_{3}\right)=(1,0)$. Let $f: E(H) \mapsto \mathbb{Z}_{2} \times \mathbb{Z}_{2}^{*}$ be $f(e)=f^{\prime}(e)$, for any $e \in E(H)$. Then

$$
\partial f(v)= \begin{cases}\partial f^{\prime}(v)-(0,1)=b^{\prime}(v)-(0,1)=b(v), & \text { if } v=v_{1}, \\ \partial f^{\prime}(v)+(1,0)=b^{\prime}(v)+(1,0)=b(v), & \text { if } v=v_{4} \\ b(v), & \text { otherwise }\end{cases}
$$

That is, for any $v \in V(H), \partial f(v)=b(v)$. Therefore by the definition of $\mathbb{Z}_{2} \times \mathbb{Z}_{2}$-connectedness, $H \in\langle A\rangle$.

By Case 1 and Case 2, we prove that if $G \in\langle A\rangle$, then $H \in\langle A\rangle$. 


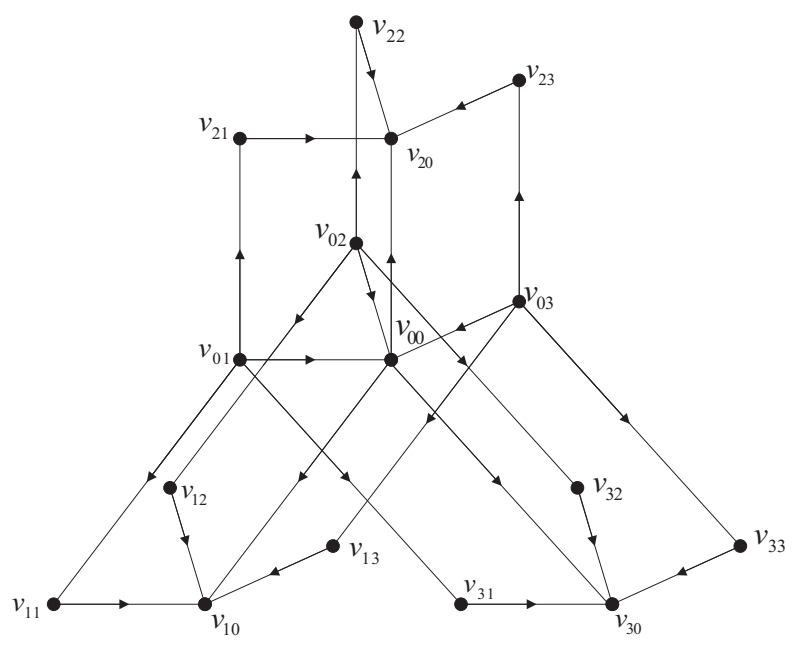

Figure $2.3 K_{1,3} \times K_{1,3}$

Lemma 2.3.3 Each of the following holds.

(i) Let $G$ be a tree. Then $\Lambda_{g}\left(G \times K_{2}\right)=5$.

(ii) Let $m \geq 2, n \geq 2$. Then $\Lambda_{g}\left(K_{1, m} \times K_{1, n}\right)=5$.

Proof. (i) If we can prove that $G \times K_{2}$ is not $\mathbb{Z}_{4}$-connected, then, by ( 2.4), $\Lambda_{g}\left(G \times K_{2}\right)=5$. We will prove by induction on $|V(G)|$ that $G \times K_{2}$ is not $\mathbb{Z}_{4}$-connected.

If $|V(G)|=2$, then $G \cong K_{2}$, and so $G \times K_{2} \cong C_{4}$. By Proposition 1.3.7, $G \times K_{2}$ is not $\mathbb{Z}_{4}$-connected. Assume that for smaller values of $|V(G)|$, if $G$ is a tree, then $G \times K_{2}$ is not $\mathbb{Z}_{4}$-connected. Assume now $|V(G)| \geq 3$. Since $G$ is a tree, $G$ has an edge $u v$ such that $u$ has degree 1 in $G$. It follows by assumption that $(G-u) \times K_{2}$ is not $\mathbb{Z}_{4}$-connected. By the definition of Cartesian product, $G \times K_{2}$ is a parallel connection of $(G-u) \times K_{2}$ and $G[\{u, v\}] \times K_{2} \cong C_{4}$. It follows by Lemma 2.3.2 that $G \times K_{2}$ is not $\mathbb{Z}_{4}$-connected. This completes the proof of (i).

(ii) By (2.4), if we can prove that $K_{1, m} \times K_{1, n}$ is not $\mathbb{Z}_{4}$-connected, then $\Lambda_{g}\left(K_{1, m} \times K_{1, n}\right)=5$. By contradiction, we assume that $K_{1, m} \times K_{1, n}$ is $\mathbb{Z}_{4}$-connected.

Suppose $V\left(K_{1, m}\right)=\left\{x_{0}, x_{1}, \ldots, x_{m}\right\}$ with $d_{K_{1, m}}\left(x_{0}\right)=m$ and $V\left(K_{1, n}\right)=\left\{y_{0}, y_{1}, \ldots, y_{n}\right\}$ with $d_{K_{1, n}}\left(y_{0}\right)=n$. Let $I=\{1,2, \ldots, m\}, I_{0}=\{0,1,2, \ldots, m\}, J=\{1,2, \ldots, n\}$ and $J_{0}=$ $\{0,1,2, \ldots, n\}$. By the definition of Cartesian product, $V\left(K_{1, m} \times K_{1, n}\right)=\left\{v_{i j}=x_{i} y_{j}\right.$ : for $i \in I_{0}$ and $\left.j \in J_{0}\right\}$. Let $E_{1}=\left\{v_{0 j} v_{i j}\right.$ : for $\left.i \in I, j \in J_{0}\right\}$ and $E_{2}=\left\{v_{i 0} v_{i j}\right.$ : for $\left.i \in I_{0}, j \in J\right\}$. Then $E\left(K_{1, m} \times K_{1, n}\right)=E_{1} \bigcup E_{2}$ and $v_{i j}$ has degree 2 , for $i \in I$ and $j \in J$.

Let $D$ be an orientation of $K_{1, m} \times K_{1, n}$ such that $v_{0 j} v_{i j} \in E_{1}$ is directed from $v_{0 j}$ to $v_{i j}$; $v_{i 0} v_{i j} \in E_{2}$ is directed from $v_{i j}$ to $v_{i 0}$ (see $K_{1,3} \times K_{1,3}$ in Figure 2.3).

Let $b: V\left(K_{1, m} \times K_{1, n}\right) \mapsto \mathbb{Z}_{4}$ such that 


$$
b(v)= \begin{cases}1, & \text { if } v=v_{01} \\ 3, & \text { if } v=v_{10} \\ 0, & \text { otherwise }\end{cases}
$$

Then $b \in Z\left(K_{1, m} \times K_{1, n}, \mathbb{Z}_{4}\right)$.

Let $\bar{f}: K_{1, m} \times K_{1, n} \mapsto \mathbb{Z}_{4}$ such that

$$
\bar{f}(e)= \begin{cases}1, & \text { if } e=v_{01} v_{00}, v_{i j} v_{i 0}, \text { for } i \in I, j \in J, \\ 3, & \text { if } e=v_{10} v_{00}, v_{i j} v_{0 j}, \text { for } i \in I, j \in J, \\ 2, & \text { otherwise. }\end{cases}
$$

By Proposition 1.3.1, there is an $f \in F\left(K_{1, m} \times K_{1, n}, \mathbb{Z}_{4}\right)$ such that $\partial f=b$, and $\bar{f}(e) \neq f(e)$, for any $e \in E\left(K_{1, m} \times K_{1, n}\right)$. For $v_{i j}$, where $i \in I$ and $j \in J$, since $b\left(v_{i j}\right)=f\left(v_{i j} v_{i 0}\right)-f\left(v_{i j} v_{0 j}\right)=$ $0, f\left(v_{i j} v_{i 0}\right)=f\left(v_{i j} v_{0 j}\right)$. Together with $f\left(v_{i j} v_{i 0}\right) \neq \bar{f}\left(v_{i j} v_{i 0}\right)=1$ and $f\left(v_{i j} v_{0 j}\right) \neq \bar{f}\left(v_{i j} v_{i 0}\right)=3$, we have

$$
f\left(v_{i j} v_{i 0}\right), f\left(v_{i j} v_{0 j}\right) \in\{0,2\} \text {, for } i \in I \text { and } j \in J \text {. }
$$

For vertex $v_{i 0}, i=2, \ldots, m$, since $b\left(v_{i 0}\right)=0=-\sum_{j=1}^{n} f\left(v_{i j} v_{i 0}\right)-f\left(v_{i 0} v_{00}\right), f\left(v_{i 0} v_{00}\right)=$ $-\sum_{j=1}^{n} f\left(v_{i j} v_{i 0}\right)$. By $(2.5), f\left(v_{i 0} v_{00}\right) \in\{0,2\}$, and since $f\left(v_{i 0} v_{00}\right) \neq \bar{f}\left(v_{i 0} v_{00}\right)=2, f\left(v_{i 0} v_{i 0}\right)=0$, for $i=2, \ldots, m$. By the similar argument, $f\left(v_{00} v_{0 j}\right)=0$, for $j=2, \ldots, n$. That is

$$
f\left(v_{i 0} v_{00}\right)=0, \text { for } i=2, \ldots, m ; f\left(v_{00} v_{0 j}\right)=0, \text { for } j=2, \ldots, n \text {. }
$$

Since $b\left(v_{00}\right)=0$, by $(2.6), f\left(v_{10} v_{00}\right)=f\left(v_{01} v_{00}\right)$. And $f\left(v_{10} v_{00}\right) \neq \bar{f}\left(v_{10} v_{00}\right)=3, f\left(v_{01} v_{00}\right) \neq$ $\bar{f}\left(v_{01} v_{00}\right)=1$, so $f\left(v_{10} v_{00}\right), f\left(v_{01} v_{00}\right) \in\{0,2\}$. For vertex $v_{10}, b\left(v_{10}\right)=3=-\sum_{j=1}^{n} f\left(v_{1 j} v_{10}\right)-$ $f\left(v_{10} v_{00}\right)$. But by $(2.5)$ and $(2.6),-\sum_{j=1}^{3} f\left(v_{1 j} v_{10}\right)-f\left(v_{10} v_{00}\right) \in\{0,2\}$. This is a contradiction. Therefore $K_{1, m} \times K_{1, n}$ is not $\mathbb{Z}_{4}$-connected.

Thus by $(2.4), \Lambda_{g}\left(K_{1, m} \times K_{1, n}\right)=5$.

Lemma 2.3.4 Each of the following holds.

(i) $\Lambda_{g}\left(P_{2} \times P_{3}\right) \leq 4$.

(ii) Let $n \geq 3$. Then $\Lambda_{g}\left(C_{n} \times K_{2}\right) \leq 4$.

(iii)If one of $G_{1}$ and $G_{2}$ is not a tree, then $\Lambda_{g}\left(G_{1} \times G_{2}\right) \leq 4$.

Proof.(i) We label most of the vertices of $P_{2} \times P_{3}$ as in Figure 2.4 a. Let $\pi_{1}=\left\langle\left\{v_{3}, v_{3}^{\prime}\right\},\left\{v_{4}, v_{4}^{\prime}\right\}\right\rangle$ and $H_{1}=\left(P_{2} \times P_{3}\right) / \pi_{1}$ (see Figure $2.4 b$ ). Let $\pi_{2}=\left\langle\left\{v_{1}, v_{1}^{\prime}\right\},\left\{v_{2}, v_{2}^{\prime}\right\}\right\rangle$ and $H_{2}=H_{1} / \pi_{2}$ (see Figure $2.5 a$ ). Let $\pi_{3}=\left\langle\left\{v_{3}^{\prime}, v_{3}^{\prime \prime}\right\},\left\{v_{4}^{\prime}, v_{4}^{\prime \prime}\right\}\right\rangle$ and $H_{3}=H_{2} / \pi_{3}$ (see Figure $2.5 b$ ). If we redraw 


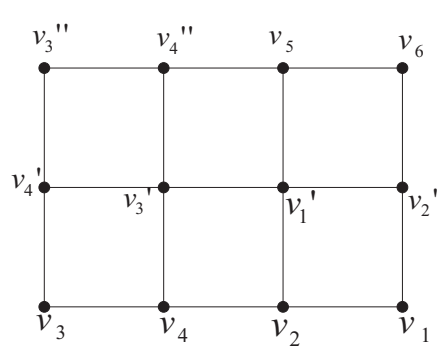

$a$

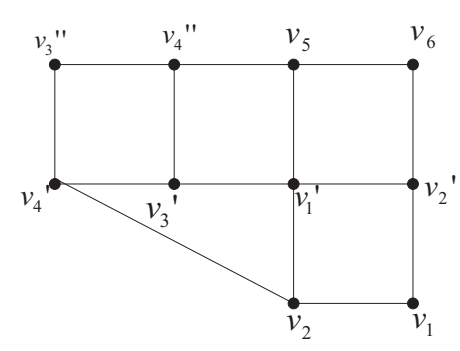

$b$

Figure 2.4

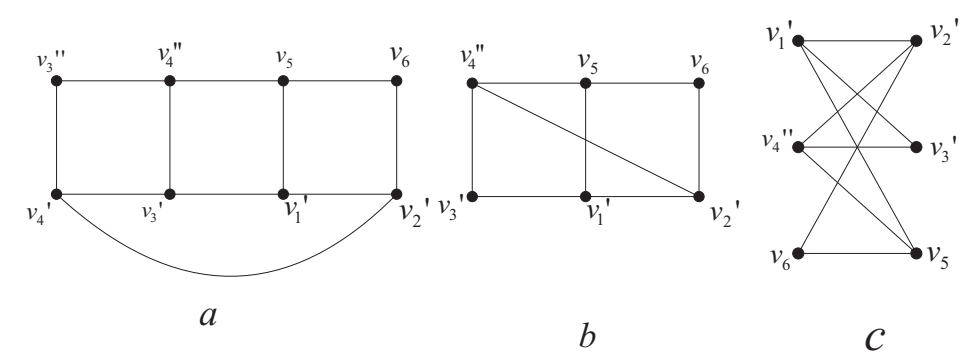

Figure 2.5

$H_{3}$ (see Figure $2.5 \mathrm{c}$ ), then $H_{3} \cong K_{3,3}-e$. By Theorem 3.1.2(iii), $H_{3} \in \mathcal{C L}$. It follows by Theorem 3.1.3 that $H_{2} \in \mathcal{C L}$. Similarly by Theorem 3.1.3, $H_{1} \in \mathcal{C L}$ and $P_{2} \times P_{3} \in \mathcal{C L}$. Then by Theorem 3.1.2(iv) and ( 2.4), $\Lambda_{g}\left(P_{2} \times P_{3}\right) \leq 4$.

(ii) First we will prove by induction on $n$ that $C_{n} \times K_{2} \in \mathcal{C L}$. When $n=3$, by Proposition 3.1.2(iii), $C_{3} \in \mathcal{C L}$, and by Lemma 2.3.1(iii), $C_{3} \times K_{2} \in \mathcal{C L}$. When $n=4$, let $C=v_{1} v_{2} v_{3} v_{4}$ be a 4-circuit in $C_{4} \times K_{2}$ (see Figure $2.6 a$ ). Let $\pi=\left\langle\left\{v_{1}, v_{3}\right\},\left\{v_{2}, v_{4}\right\}\right\rangle$ and $G^{\prime}=\left(C_{4} \times K_{2}\right) / \pi$ (see Figure $2.6 \mathrm{~b}$ ). If we redraw $G^{\prime}$ (see Figure $2.6 \mathrm{c}$ ), then $G^{\prime} \cong K_{3,3}$. It follows by Proposition 3.1.2(iii) that $G^{\prime}$ is collapsible. Therefore by Lemma 3.1.3, $C_{4} \times K_{2} \in \mathcal{C L}$.

For a fixed $n>4$, assume that for any $m<n, C_{m} \times K_{2} \in \mathcal{C L}$. Let $C=v_{1} v_{2} v_{1}^{\prime} v_{2}^{\prime}$ and $C^{\prime}=$ $v_{1}^{\prime} v_{2} v_{1}^{\prime \prime} v_{2}^{\prime \prime}$ be two 4-circuits contained in $C_{n} \times K_{2}$ (see Figure $2.7 a$ ). Let $\pi_{1}=\left\langle\left\{v_{1}, v_{1}^{\prime}\right\},\left\{v_{2}, v_{2}^{\prime}\right\}\right\rangle$ and $\pi_{2}=\left\langle\left\{v_{1}, v_{1}^{\prime \prime}\right\},\left\{v_{2}, v_{2}^{\prime \prime}\right\}\right\rangle$. Let $G^{\prime}=\left(C_{n} \times K_{2}\right) / \pi_{1}$ (see Figure 2.7 a) and $G^{\prime \prime}=\left(C_{n} \times K_{2}\right) / \pi_{1} / \pi_{2}$ (see Figure $2.7 \mathrm{~b}$ ). Then by assumption $\left(C_{n} \times K_{2}\right) / \pi_{1} / \pi_{2} \cong C_{n-2} \times K_{2} \in \mathcal{C L}$ (see Figure 2.7 $c)$. By Lemma $3.1 .3,\left(C_{n} \times K_{2}\right) / \pi_{1} \in \mathcal{C L}$. And by Lemma 3.1 .3 again, $\left(C_{n} \times K_{2}\right) \in \mathcal{C L}$. Thus $C_{n} \times K_{2} \in \mathcal{C L}$, for $n \geq 3$. It follows by (2.4) and Theorem 3.1.2 (iv) that $\Lambda_{g}\left(C_{n} \times K_{2}\right) \leq 4$, for $n \geq 3$.

(iii) Suppose $G_{1}$ is not a tree, then there is a circuit $C_{n} \subseteq G_{1}$, where $n \geq 3$. Therefore $G_{1} \times G_{2}$ 


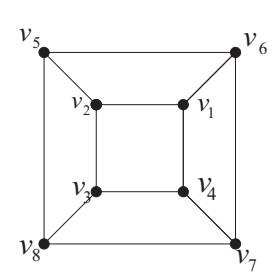

$a$

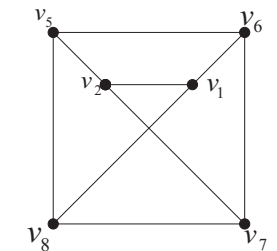

$b$

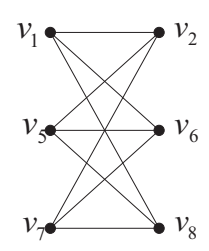

C

Figure 2.6

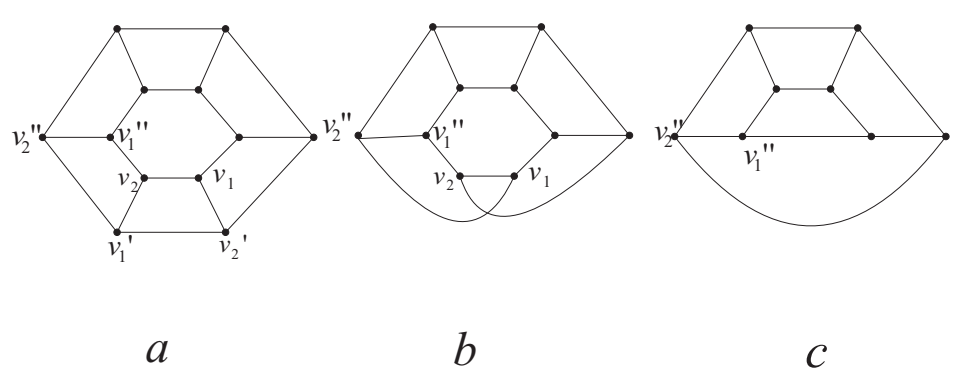

Figure 2.7

contains a nontrivial collapsible subgraph $H \cong C_{n} \times K_{2}$. It follows by Theorem 2.3.1(iii) that $\Lambda_{g}\left(G_{1} \times G_{2}\right) \leq 4$.

Proof of Theorem 2.1.6: By ( 2.4) and by Lemma 2.3.3, we may assume that $\Lambda_{g}\left(G_{1} \otimes G_{2}\right)=5$ to prove that either $G_{1} \cong K_{1, m}$ and $G_{2} \cong K_{1, n}$, where $n, m \geq 2$ or $G_{1}$ is a tree and $G_{2} \cong K_{2}$.

If $G_{1}$ or $G_{2}$ has a circuit, then by Lemma 2.3.4(iii), $\Lambda_{g}\left(G_{1} \otimes G_{2}\right) \leq 4$. Hence we may assume that both $G_{1}$ and $G_{2}$ are trees.

Case 1: If $\min \left\{\left|V\left(G_{1}\right)\right|,\left|V\left(G_{2}\right)\right|\right\}=2$, assume $V\left(G_{2}\right)=2$. Since $G_{2}$ is connected, $G_{2} \cong K_{2}$, by Lemma 2.3.3(ii), $\Lambda_{g}\left(G_{1} \times G_{2}\right)=5$.

Case 2: If $\min \left\{\left|V\left(G_{1}\right)\right|,\left|V\left(G_{2}\right)\right|\right\} \geq 3$, then since $G_{1}$ and $G_{2}$ are connected, both $G_{1}$ and $G_{2}$ contain a path of length 2 . If one of $G_{1}$ and $G_{2}$ contains a path of length 3 , then it follows by Lemma 2.3.4(i) that $G_{1} \otimes G_{2}$ has a nontrivial subgraph $H \cong P_{2} \times P_{3}$ with $\Lambda_{g}(H) \leq 4$, and so by (2.1) and by Theorem 2.3.1(iii), $\Lambda_{g}\left(G_{1} \otimes G_{2}\right) \leq 4$. Therefore $G_{1}$ and $G_{2}$ contains only paths with length 2 . So $G_{1} \cong K_{1, m}$ and $G_{2} \cong K_{1, n}$, for $m, n \geq 2$. 


\section{Chapter 3}

\section{Group Connectivity in Line Graphs}

\subsection{Introduction}

Given an $f \in F(G, A)$ and a subset $X \in E(G),\left.f\right|_{X}$ denotes the restriction of $f$ to $X$. For $b \in Z(G, A)$, a graph $G$ is $(A, b)$-extensible from $v$, if for all $f^{\prime}: E(v) \mapsto A^{*}$ satisfying $\partial f^{\prime}(v)=b(v)$, there exists an $f \in F^{*}(G, A)$ with $\partial f=b$ such that $\left.f\right|_{E(v)}=f^{\prime}$. If for any $b \in Z(G, A), G$ is $(A, b)$-extensible from $v$, then $G$ is called $A$-extensible from $v$. By definition, if $G$ is $A$-extensible from $v$, then $G \in\langle A\rangle$.

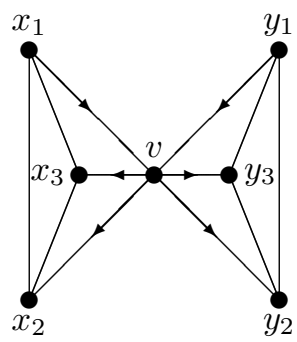

$(a): H$

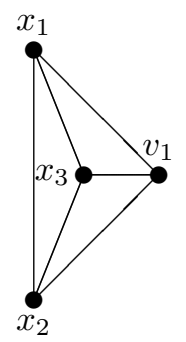

(b)

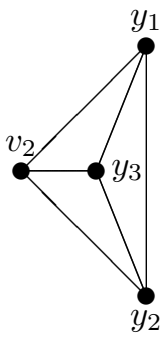

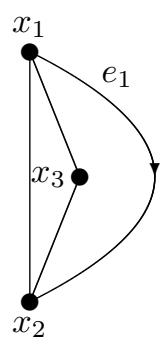

$(c): G_{v}$

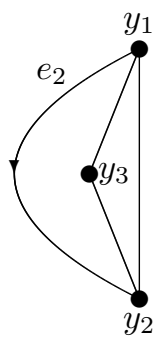

3.1.1

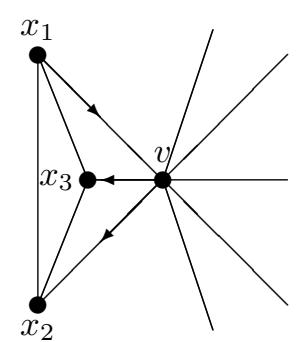

(a): $H$

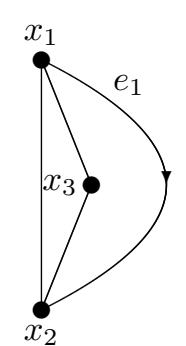

$(c): G_{v}$

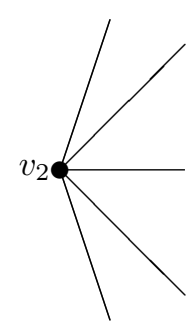

Figure 3.2: $\quad$ Reduction in Lemma 3.1.1

Lemma 3.1.1 (Lemma 2.3, [27]) Let $G$ be a graph and $H \cong K_{4}$ be a subgraph of $G$ and 
$v \in V(H)$ (see Figure 3.1(a) and Figure 3.2(a)). If $d_{G}(v)=6$ and if $G$ has another subgraph $H^{\prime} \cong K_{4}$ such that $V(H) \cap V\left(H^{\prime}\right)=\{v\}, N_{H}(v)=\left\{x_{1}, x_{2}, x_{3}\right\}$ and $N_{H^{\prime}}(v)=\left\{y_{1}, y_{2}, y_{3}\right\}$, then let $G_{v}$ be the graph obtained from $G$ by splitting the vertex $v \in V(G)$ into $v_{1}, v_{2}$ (as depicted in Figure 3.1(b)), and by first deleting $x_{3} v_{1}, y_{3} v_{2}$ and then contracting $v_{1} x_{1}, v_{2} y_{1}$ (depicted in Figure 3.1 $(c))$; and if $d_{G}(v)>6$, then let $G_{v}$ be the graph obtained from $G$ by splitting the vertex $v \in V(G)$ into $v_{1}, v_{2}$, deleting the edge $v_{1} x_{3}$, and then contracting $v_{1} x_{1}$ (depicted in Figure 3.2(c)).

(i) If $G_{v} \in\left\langle\mathbb{Z}_{3}\right\rangle$, then $G \in\left\langle\mathbb{Z}_{3}\right\rangle$.

(ii) If for some $u \in V(G)-v, G_{v}$ is $\mathbb{Z}_{3}$-extensible from $u$, then $G$ is also $\mathbb{Z}_{3}$-extensible from $u$.

Proof. The proof for (i) is given in [27]. The proof for (ii) is similar to that for (i) and so omitted.

Definition 3.1.2 Suppose that $N_{G}(v)=\left\{v_{1}, v_{2}, \ldots, v_{n}\right\}$, and let $Y=\left\{v v_{1}, v v_{2}\right\}$. As in [28], define $G_{[v, Y]}$ to be the graph obtained from $G-\left\{v v_{1}, v v_{2}\right\}$ by adding a new edge that joins $v_{1}$ and $v_{2}$.

Lemma 3.1.3 (Lemma 6, [28]): For any Abelian group $A$ and $b \in Z(G, A)$, if $G_{[v, Y]}$ has an $(A, b)-N Z F$, then $G$ has an $(A, b)-N Z F$. Moreover, if $G_{[v, Y]}$ is A-extensible from a vertex $u$ with $u \neq v$, then $G$ is also $A$-extensible from $u$.

Lemma 3.1.4 (Lemma 7, [28]): Let $A$ be an Abelian group, $G$ be a graph and $H \in\langle A\rangle$ be a subgraph of $G$. We define $G^{*}=G / H$ and denote by $v_{H}$ the vertex in $G^{*}$ onto which $H$ is contracted. For any $b \in Z(G, A)$, define $b^{\prime}: V\left(G^{*}\right) \mapsto A$ by $b^{\prime}\left(v_{H}\right)=\sum_{u \in V(H)} b(u)$ and $b^{\prime}(v)=b(v)$ for $v \neq v_{H}$. If $G^{*}$ admits an $\left(A, b^{\prime}\right)-N Z F f^{*}$, then $f^{*}$ can be extended to an $(A, b)-N Z F$ of $G$.

\subsection{Ryjácěk ([32]) Closure of a Claw-free Graph}

We shall follow [6] to define a line graph. The line graph of a graph $G$, denoted by $L(G)$, has $E(G)$ as its vertex set, where for an integer $k \in\{0,1,2\}$, two vertices in $L(G)$ are joined by $k$ edges in $L(G)$ if and only if the corresponding edges in $G$ are sharing $k$ common vertices in $G$. In other words, if $e_{1}$ and $e_{2}$ are adjacent but not parallel in $G$, then $e_{1}$ and $e_{2}$ are joined by one edge in $L(G)$; if $e_{1}$ and $e_{2}$ are parallel edges in $G$, then $e_{1}$ and $e_{2}$ are joined by two (parallel) edges in $L(G)$. Note that our definition for line is slightly different from the one defined in [2] (called an edge graph there). But when $G$ is a simple graph, both definitions are the same. The main reason for us to adopt this definition in [6] instead of the traditional definition of a line graph is explained in the introduction section of [27]. 
For an integer $i>0$ and for a graph $G$, define

$$
D_{i}(G)=\left\{v \in V(G): d_{G}(v)=i\right\} .
$$

A vertex $v \in V(G)$ is locally connected if $G\left[N_{G}(v)\right]$ is connected. A graph $G$ is claw-free if $G$ does not have an induced subgraph isomorphic to $K_{1,3}$. It is well known ([1], [31]) that every line graph is a claw-free graph.

Following the definition of Ryjácěk ([32]), a graph $H$ is the closure of a claw-free graph $G$, denoted by $H=\operatorname{cl}(G)$, if

(CL1) there is a sequence of graphs $G_{1}, \ldots, G_{t}$ such that $G_{1}=G, G_{t}=H, V\left(G_{i+1}\right)=V\left(G_{i}\right)$ and $E\left(G_{i+1}\right)=E\left(G_{i}\right) \bigcup\left\{u v: u, v \in N_{G_{i}}\left(x_{i}\right), u v \notin E\left(G_{i}\right)\right\}$ for some $x_{i} \in V\left(G_{i}\right)$ with connected non-complete $G_{i}\left[N_{G_{i}}\left(x_{i}\right)\right]$, for $i=1, \ldots, t-1$, and

(CL2) No vertex of $H$ has a connected non-complete neighborhood.

Lemma 3.2.1 Let $G$ be a claw-free graph.

(i) For any $v \in V(G)$, either $G\left[N_{G}(v)\right]$ is an edge disjoined union of two cliques or $v$ is a locally connected vertex.

(ii) If $v$ is a locally connected vertex of $G$, then $G\left[N_{G}[v]\right]$ is triangularly connected.

Proof. (i) follows from the definition of claw-free graphs immediately.

(ii) Let $e=x y, e^{\prime}=u w \in E\left(G\left[N_{G}[v]\right]\right)$, where $y, w \in N_{G}(v)$ and $e$ and $e^{\prime}$ are not contained in the same triangle. Since $v$ is locally connected, there is a path $P=v_{1} v_{2} \ldots v_{s}$ joining $y=v_{1}$ and $w=v_{s}$, where $v_{i} \in N_{G}(v)$, for $i=2, \ldots, s-1$, in such a way that if $x \neq v$, then $x=v_{2}$, and if $u \neq v$, then $u=v_{s-1}$. Since $v v_{i} \in E(G)$, and since $e$ is in the 3-circuit $G\left[\left\{v, v_{1}, v_{2}\right\}\right]$ and $e^{\prime}$ is in the 3-circuit $G\left[\left\{v, v_{s-1}, v_{s}\right\}\right]$, the 3-circuits $G\left[\left\{v, v_{i}, v_{i+1}\right\}\right], 1 \leq i \leq s-1$, is an $\left(e, e^{\prime}\right)$-triangle-path. Therefore $G\left[N_{G}(v)\right]$ is triangularly connected.

Theorem 3.2.2 The following are equivalent.

(i) Every 5-edge-connected graph is $\mathbb{Z}_{3}$-connected.

(ii) Every 5-edge-connected line graph is $\mathbb{Z}_{3}$-connected.

Proof. As (i) trivially implies (ii), it suffices to show that (ii) implies (i). Let $G$ be a graph with $\kappa^{\prime}(G) \geq 5$ and let $S(G)$, the subdivided graph of $G$, be the graph obtained from $G$ by replacing each edge $e=u v$ of $G$ by a 2-path $u v_{e} v$, where $v_{e}$ is a new vertex. Let $e^{\prime}$ be the edge in $L(S(G))$ that has $u v_{e}$ and $v_{e} v$ as its ends, and let $E^{\prime}=\left\{e^{\prime} \in E(L(S(G))) \mid e \in E(G)\right\}$. It then follows that $L(S(G)) /\left[E(L(S(G)))-E^{\prime}\right]=G$. (See Claims 1 and 2 within the proof of Theorem 3.4 in [6]). Moreover, If $\kappa^{\prime}(G) \geq 5$, then $\kappa^{\prime}(L(S(G))) \geq 5$, and so $L(S(G)) \in\left\langle\mathbb{Z}_{3}\right\rangle$ follows by (ii). As $L(S(G)) /\left[E(L(S(G)))-E^{\prime}\right]=G$, by Proposition1.3.1(C2), $G \in\left\langle\mathbb{Z}_{3}\right\rangle$, and so (i) must hold. 


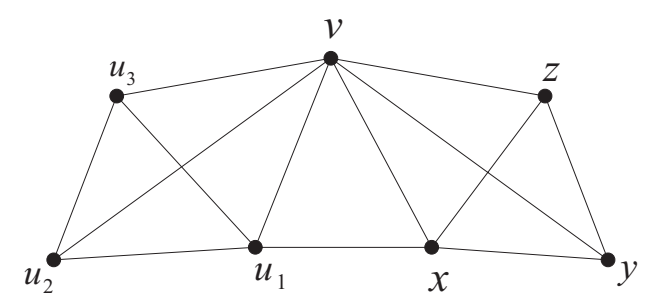

Figure 3.3 The graph $L_{1}$ in Lemma 3.2.5

Theorem 3.2.3 Let $A$ be an Abelian group with $|A| \geq 4$ and $G$ be a claw-free graph with $\delta(G) \geq 3$. Each of the following holds:

(i) Suppose that a vertex $v \in V(G)$ is locally connected, and $x, y \in N_{G}(v)$ are not adjacent. If $G+x y$ is A-connected, then $G$ is A-connected.

(ii) If $\operatorname{cl}(G)$ is A-connected, then $G$ is A-connected.

Proof. By the definition of the closure of a claw-free graph, $\operatorname{cl}(G)$ contains $G$ as a spanning connected subgraph. Thus Theorem3.2.3(ii) follows from Theorem3.2.3(i) and Lemma1.3.3(i). Therefore, it suffices to prove Theorem3.2.3(i).

Let $G$ be a claw-free graph and let $v \in V(G)$ be a locally connected vertex. By Lemma 3.2.1(ii), every edge in the graph $G\left[N_{G}[v]\right]$ lies in a 3-circuit. As $|A| \geq 4$, by Lemma 1.3.3(ii) with $n=3$, every edge of $G\left[N_{G}[v]\right]$ lies in an $A$-connected subgraph of $G\left[N_{G}[v]\right]$. It follows by Lemma 1.3.3(i) that $G\left[N_{G}[v]\right] \in\langle A\rangle$. Let $G^{\prime}=G+x y$. Then $G^{\prime}\left[N_{G^{\prime}}[v]\right]=G\left[N_{G}[v]\right]+x y$. As $G\left[N_{G}[v]\right] \in\langle A\rangle$, it follows by Lemma 1.3.3(i) that $G^{\prime}\left[N_{G^{\prime}}[v]\right] \in\langle A\rangle$. Hence if $G^{\prime} \in\langle A\rangle$, then by Proposition 1.3.1(C2), $G^{\prime} / G^{\prime}\left[N_{G^{\prime}}[v]\right] \in\langle A\rangle$. As $G / G\left[N_{G}[v]\right]=G^{\prime} / G^{\prime}\left[N_{G^{\prime}}[v]\right] \in\langle A\rangle$, and as $G\left[N_{G}[v]\right] \in\langle A\rangle$, it follows by Proposition 1.3.1(C3) that $G \in\langle A\rangle$.

Lemma 3.2.4 Let $G$ be a claw-free graph with $\delta(G) \geq 3$ and $v \in V(G)$ be locally connected. Then $G\left[N_{G}(v)\right]$ has a Hamilton path.

Proof. Arguing by contradiction, we assume that $G\left[N_{G}(v)\right]$ does not have a Hamilton path. As every connected graph on three vertices has a Hamilton path, we assume $d_{G}(v) \geq 4$.

Let $P=x_{1} x_{2} \ldots x_{p}$ be a longest path in $G\left[N_{G}(v)\right]$. As $V(P) \neq N_{G}(v)$, we can pick $x \in$ $N_{G}(v)-V(P)$. As $P$ is longest, $x x_{1}, x x_{p} \notin E(G)$. Since $G\left[\left\{x, x_{1}, x_{p}, v\right\}\right] \nsucceq K_{1,3}$, we must have $x_{1} x_{p} \in E(G)$. Since $G\left[N_{G}(v)\right]$ is connected, $G\left[N_{G}(v)\right]$ has a path $P^{\prime}$ from $x$ to a vertex $x_{i_{0}} \in V(P)$, internally disjoint from $V(P)$. It follows that $x P^{\prime} x_{i_{0}} x_{i_{0}+1} \ldots x_{p} x_{1} x_{2} \ldots x_{i_{0}-1}$ is a longer path, contrary to the assumption that $P$ is a longest path in $G\left[N_{G}(v)\right]$.

Lemma 3.2.5 Let $G$ be a claw-free graph with $\delta(G) \geq 6$ and $v \in V(G)$ be a locally connected vertex. Each of the following holds. 
(i) If $d_{G}(v) \geq 6$ and if $G\left[N_{G}[v]\right] \in\langle W F\rangle$, then $G\left[N_{G}[v]\right]$ contains the graph $L_{1}$ depicted in Figure 3 as an induced subgraph. Moreover, if $d_{G}(v)=6$, then $G\left[N_{G}[v]\right]=L_{1}$.

(ii) If $d_{G}(v) \geq 7$, then $G\left[N_{G}[v]\right]$ is $\mathbb{Z}_{3}$-connected.

Proof. (i) Suppose $d_{G}(v)=m \geq 6$. By Lemma 3.2.4, $G\left[N_{G}(v)\right]$ has a path $P=v_{1} v_{2} \ldots v_{m}$, where $v_{i} \in N_{G}(v), 1 \leq i \leq m$.

We claim that $G\left[N_{G}[v]\right]$ has a $K_{4}$ with $v \in V\left(K_{4}\right)$. If not, then $L=G\left[\left\{v, v_{1}, v_{3}, v_{5}\right\}\right] \nsucceq K_{4}$, and so both $v_{1} v_{3} \notin E(G)$ and $v_{3} v_{5} \notin E(G)$. Since $G\left[\left\{v, v_{1}, v_{3}, v_{5}\right\}\right] \nRightarrow K_{1,3}$, we must have $v_{1} v_{5} \in$ $E(G)$. Similarly, $v_{2} v_{6} \in E(G)$ as $G\left[\left\{v, v_{2}, v_{4}, v_{6}\right\}\right] \nRightarrow K_{4}$. It follows that $G\left[\left\{v, v_{1}, v_{2}, v_{5}, v_{6}\right\}\right]$ consists a $W_{4}$, contrary to Corollary 1.3 .7 as $G\left[N_{G}[v]\right] \in\langle W F\rangle$. Thus $G\left[N_{G}[v]\right]$ must have a $K_{4}$.

Let $H_{1} \cong K_{4}$ be a subgraph of $G\left[N_{G}[v]\right]$ with $v \in V\left(H_{1}\right)$. Let $W=N_{G}(v)-V\left(H_{1}\right)$. Note that for all $w \in W$, if $w$ is adjacent to two vertices in $V\left(H_{1}\right)-\{v\}$, then $W_{4} \subseteq G\left[V\left(H_{1}\right) \cup\{w\}\right]$, contrary to Corollary 1.3.7. Since $|W| \geq 3$, and since every $w \in W$ is adjacent to at most one vertex in $V\left(H_{1}\right)$, it follows from the fact that $P$ is a Hamilton path that there must be $x, y, z \in W$ such that $x z, y z \in E(G)$. Let $V\left(H_{1}\right)-\{v\}=\left\{u_{1}, u_{2}, u_{3}\right\}$. With these notations, we further claim that $K_{3} \subseteq G[W]$.

Assume that $G[W]$ contains no $K_{3}$ 's. Then $x y \notin E(G)$. Since for all $u_{i} \in V\left(H_{1}\right)-$ $\{v\}, G\left[\left\{v, x, y, u_{i}\right\}\right] \nRightarrow K_{1,3}, u_{i}$ must be adjacent to $x$ or $y$. Hence we may assume that there are two $u_{i}^{\prime} \mathrm{s}$, say $u_{1}, u_{2}$, that are adjacent to a same vertex in $\{x, y\}$, say $x$. It follows that $G\left[\left\{v, u_{1}, u_{2}, u_{3}, x\right\}\right]$ contains a $W_{4}$, contrary to Corollary 1.3.7. Thus we must have both $G[\{x, y, z\}] \cong K_{3}$ and $G[\{v, x, y, z\}] \cong K_{4}$. Let $H_{2}=G[\{v, x, y, z\}]$.

Now assume that $d_{G}(v)=6$, and so $N_{G}(v)=V\left(H_{1}\right) \cup W$. Since $v$ is locally connected, $G\left[N_{G}(v)\right]$ has an edge $e$, say $e=u_{1} x$, joining $H_{1}$ and $H_{2}$. Let $G^{\prime}=G\left[E\left(H_{1}\right) \cup E\left(H_{2}\right) \cup\{e\}\right]$. Then $G^{\prime} \subseteq G\left[N_{G}[v]\right]$. By the definition of $\langle W F\rangle, G^{\prime} \in\langle W F\rangle$. Let $e^{\prime} \in E\left(G\left[N_{G}[v]\right]\right)-E\left(G^{\prime}\right)$. If $e$ and $e^{\prime}$ are not adjacent, say $e^{\prime}=u_{2} y$, then $W_{4} \subseteq G\left[\left\{v, u_{1}, u_{2}, x, y\right\}\right]$; if $e$ and $e^{\prime}$ are adjacent, say $e^{\prime}=u_{2} x$, then $W_{4} \subseteq G\left[\left\{v, u_{1}, u_{2}, u_{3}, x\right\}\right]$, contrary to Corollary 1.3.7 in either case. Thus we must have $G\left[N_{G}[v]\right]=G^{\prime}$, as desired.

(ii) By contradiction, assume that $G\left[N_{G}[v]\right] \notin\left\langle\mathbb{Z}_{3}\right\rangle$. By Lemma 2.2.1(ii), $G\left[N_{G}[v]\right]$ is triangularly connected. By Theorem 1.3.6, $G\left[N_{G}[v]\right] \in\langle W F\rangle$.

By (i), $G\left[N_{G}[v]\right]$ contains a subgraph $L_{1}$ as depicted in Figure 3. Define $H_{1}$ and $H_{2}$ as the two 4-cliques above in $G\left[N_{G}[v]\right]$ with $V\left(H_{1}\right) \cap V\left(H_{2}\right)=\{v\}$, and let $W^{\prime}=N_{G}(v)-\left(V\left(H_{1}\right) \cup V\left(H_{2}\right)\right)$. Again since $G\left[N_{G}[v]\right]$ contains no $W_{4}$, every vertex $w^{\prime} \in W^{\prime}$ is adjacent to at most one vertex in $V\left(H_{i}\right), i \in\{1,2\}$. It follows that $G\left[N_{G}[v]\right]$ contains an induced subgraph $G\left[\left\{v, w^{\prime}, z_{1}, z_{2}\right\}\right] \cong$ $K_{1,3}$, for some $z_{i} \in V\left(H_{i}\right)-\{v\},(1 \leq i \leq 2)$, contrary to the assumption that $G$ is claw-free. Thus $G\left[N_{G}[v]\right]$ must be $\mathbb{Z}_{3}$-connected if $d_{G}(v) \geq 7$.

Theorem 3.2.6 Let $G$ be a claw-free graph with $\delta(G) \geq 7$. If $\operatorname{cl}(G) \in\left\langle\mathbb{Z}_{3}\right\rangle$, then $G \in\left\langle\mathbb{Z}_{3}\right\rangle$. 
Proof. For any locally connected $v \in V(G)$ with $d_{G}(v) \geq 7$, by Lemma 3.2.5(ii), $G\left[N_{G}[v]\right]$ is $\mathbb{Z}_{3}$-connected. Let $H_{1}, \ldots, H_{m}$ be all the maximal $\mathbb{Z}_{3}$-connected subgraphs of $G$. Suppose $G_{1}=G, G_{2}, \ldots, G_{m}, G_{m+1}$ is a sequence of graphs such that, for $i=1,2,3, \ldots m, G_{i+1}=G_{i} / H_{i}$. Suppose $G_{1}^{\prime}=\operatorname{cl}(G), G_{2}^{\prime}, \ldots, G_{m}^{\prime}, G_{m+1}^{\prime}$ is a sequence of graphs such that, for $i=1,2,3, \ldots m$, $G_{i+1}^{\prime}=G_{i}^{\prime} / H_{i}^{\prime}$, where $H_{i}^{\prime}$ is the subgraph induced by $V\left(H_{i}\right)$ in $\operatorname{cl}(G)$. Note that $H_{i} \subseteq H_{i}^{\prime}$.

Now we claim that $G_{m+1}^{\prime}=G_{m+1}$. By the construction of $G_{m}$ and $G_{m}^{\prime}$, we have $V\left(G_{m+1}^{\prime}\right)=$ $V\left(G_{m+1}\right)$ and $E\left(G_{m+1}\right) \subset E\left(G_{m+1}^{\prime}\right)$. We only need to show $E\left(G_{m+1}^{\prime}\right) \subset E\left(G_{m+1}\right)$. Let $e \in$ $E\left(G_{m+1}^{\prime}\right)$ and $e \notin E\left(G_{m+1}\right)$. Assume $e=v_{1} v_{2}$ in $c l(G)$. By the definition of closure, there is a locally connected vertex $v \in V(G)$ such that $v_{1}, v_{2} \in N_{G}(v)$ and $v_{1}$ and $v_{2}$ are not adjacent. By Lemma 3.2.5(ii) $G\left[N_{G}[v]\right]$ is $Z_{3}$-connected, then $G[N[v]]$ will be contained in some $H_{i}$, and $e \in E\left(H_{i}^{\prime}\right)$, contrary to the fact that $e \in G_{m+1}^{\prime}$.

Therefore $G_{m+1}=G_{m+1}^{\prime}$. Since $\operatorname{cl}(G)=G_{1}^{\prime} \in\left\langle\mathbb{Z}_{4}\right\rangle$, by Proposition 1.3.1(C2) $G_{2}^{\prime} \in\left\langle\mathbb{Z}_{4}\right\rangle$. Inductively, we conclude that $G_{i}^{\prime} \in\left\langle\mathbb{Z}_{4}\right\rangle, 1 \leq i \leq m+1$. It follows that $G_{m+1}=G_{m+1}^{\prime} \in\left\langle\mathbb{Z}_{4}\right\rangle$. Since $H_{m} \in\left\langle\mathbb{Z}_{4}\right\rangle$, by Proposition 1.3.1(C3) $G_{m} \in\left\langle\mathbb{Z}_{4}\right\rangle$. Inductively, we conclude that $G_{i} \in\left\langle\mathbb{Z}_{4}\right\rangle$, $1 \leq i \leq m-1$. In particular, $G=G_{1} \in\left\langle\mathbb{Z}_{4}\right\rangle$. 


\section{Chapter 4}

\section{Group Connectivity of $J_{3}$ Line Graphs and $J_{3}$ claw-free Graphs}

\subsection{Introduction}

An edge cut $X$ of $G$ is essential if $G-X$ has at least two nontrivial components. For any integer $k>0$, a graph is essentially $k$-edge-connected if $G$ has no essential edge cut $X$ with $|X|<k$. By this definition, if a graph $G$ is $k$-edge-connected, then $G$ is also essentially $k$-edge-connected. An edge cut $X$ of $G$ is a cyclical edge cut if neither side of $G-X$ is acyclic; $G$ is cyclically $k$-edge-connected if $G$ has no cyclical edge cut of size less than $k$.

By the definition of a line graph, for all $v \in V(G), E(v)$ induce a complete subgraph $H_{v}$ in $L(G)$. When $u, v \in V(G)$ with $u \neq v$, if $G$ is simple, then $H_{v}$ and $H_{u}$ are edge disjoint complete subgraphs of $L(G)$. Such an observation motivates the following definition.

For a connected graph $G$, a partition $\left(E_{1}, E_{2}, \cdots, E_{k}\right)$ of $E(G)$ is a clique partition of $G$ if $G\left[E_{i}\right]$ is spanned by a maximal complete subgraph of $G$ for each $i \in\{1,2, \cdots, k\}$. Furthermore, $\left(E_{1}, E_{2}, \cdots, E_{k}\right)$ is a $(\geq 3)$-clique partition of $G$, if for each $i \in\{1,2, \cdots, k\}, G\left[E_{i}\right]$ is spanned by a $K_{n_{i}}$ with $n_{i} \geq 3$; and a $\left(K_{3}, K_{4}\right)$-partition if for each $i \in\{1,2, \cdots, k\}, G\left[E_{i}\right]$ is spanned by a maximal subgraph of $G$ isomorphic to a $K_{3}$ or a $K_{4}$. Note that if $G$ is simple, and if $\left(E_{1}, E_{2}, \cdots, E_{k}\right)$ of $E(G)$ is a clique partition of $G$, then $\left|V\left(G\left[E_{i}\right]\right) \cap V\left(G\left[E_{j}\right]\right)\right| \leq 1$ where $i \neq j$ and $i, j \in\{1,2, \cdots, k\}$. By the definition of a line graph, every $J_{3}$ line graph must have a ( $\geq 3$ )-clique partition. By Proposition 1.3.1 and Lemma 1.3.3(iv), it suffices to study the $\mathbb{Z}_{3}$-connectedness of graphs with a $\left(K_{3}, K_{4}\right)$-partition.

For an integer $m>0, m K_{2}$ denotes the graph with 2 vertices and $m$ parallel edges. Define $\mathcal{F}_{0}=\left\{G: G\right.$ has a $\left(K_{3}, K_{4}\right)$-partition $\}$, and $\mathcal{F}$ to be the family of graphs such that $G \in \mathcal{F}$ if and only if either $G \in \mathcal{F}_{0}$, or $G$ is obtained from a member $G^{\prime} \in \mathcal{F}_{0}$ by contracting some edges in $E\left(G^{\prime}\right)$. 


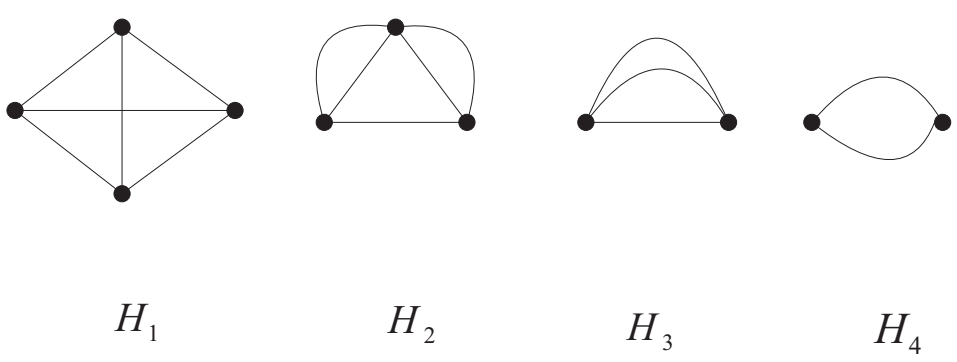

Figure $4.1 H_{0}, H_{1}, H_{2}, H_{3}, H_{4}$

Let $H_{1} \cong K_{4}$ and $H_{0}, H_{2}, H_{3}$ be contractions of $H_{1}$, where $H_{0}=4 K_{2}$. Let $H_{4} \cong 2 K_{2}$ be the graph obtained from $K_{3}$ by contracting an edge (see Figure 4.1 for $H_{i}, 0 \leq i \leq 4$ ). Then for every graph $G \in \mathcal{F}, E(G)$ is partitioned into $E_{1}, E_{2}, \ldots, E_{k}$, such that $G\left[E_{j}\right] \in$ $\left\{H_{0}, H_{1}, H_{2}, H_{3}, K_{3}, H_{4}\right\}$, for $j=1,2, \ldots, k$.

\subsection{Main Results}

Theorem 4.2.1 Each of the following holds.

(i) Every 6-edge-connected $J_{3}$ line graph is $\mathbb{Z}_{3}$-connected.

(ii) Every 7-edge-connected $J_{3}$ claw-free graph is $\mathbb{Z}_{3}$-connected.

We shall prove the following stronger result, which implies Theorem 2.3.1.

Theorem 4.2.2 Let $G \in \mathcal{F}$ be an essentially 6-edge-connected graph with $\mid D_{3}(G) \cup D_{4}(G) \cup$ $D_{5}(G) \mid \leq 1$. Each of the following holds.

(i) For any $u \in D_{6}(G) \cup D_{7}(G) \cup D_{8}(G)$, $G$ is $\mathbb{Z}_{3}$-extensible from $u$.

(ii) If $D_{6}(G) \cup D_{7}(G) \cup D_{8}(G)=\varnothing$, then $G$ is $\mathbb{Z}_{3}$-connected.

Assuming the truth of Theorem 4.2.2, we can derive the following results. A graph $G$ is $\mathbb{Z}_{3}$-reduced if $G$ does not have a nontrivial subgraph in $\left\langle\mathbb{Z}_{3}\right\rangle$.

Theorem 4.2.3 Every 6-edge-connected graph with a $(\geq 3)$-clique partition is $\mathbb{Z}_{3}$-connected.

Proof. Let $G$ be a counterexample with $|V(G)|$ minimized. As the theorem holds trivially if $|V(G)| \leq 6$, we assume that $|V(G)| \geq 7$. By the minimality of $G, G$ is $\mathbb{Z}_{3}$-reduced. By Lemma 1.3 .3 (iv), $G$ must have a $\left(K_{3}, K_{4}\right)$-partition, and so $G \in \mathcal{F}$. Thus $G \in\left\langle\mathbb{Z}_{3}\right\rangle$ by Theorem 4.2.2.

Proof of Theorem 2.3.1 (i) Let $G$ be a 6 -edge-connected $J_{3}$ line graph. By the definition of a line graph, and since $G$ is a $J_{3}$ graph, $G$ is a 6 -edge-connected graph with a ( $\geq 3$ )-clique partition. It follows by Theorem 4.2 .3 that $G$ is $\mathbb{Z}_{3}$-connected. 
(ii) Let $G$ be a 7-edge-connected $J_{3}$ claw-free graph, and let $\operatorname{cl}(G)$ be its closure. Then $c l(G)$ is a 7-edge-connected $J_{3}$ line graph. By Theorem 4.2.1(i), $\operatorname{cl}(G)$ is $\mathbb{Z}_{3}$-connected. By Theorem 3.2.6, $G$ is $\mathbb{Z}_{3}$-connected. This completes the proof of Theorem 4.2.1.

\subsection{The Proof of Theorem 4.2.2}

Throughout this section, for a graph $G$ and for $W \subseteq E(G)$, any map $g$ : $W \mapsto \mathbb{Z}_{3}$ is viewed as a map $g: E(G) \mapsto \mathbb{Z}_{3}$ such that $g(e)=0$,for all $e \in E(G)-W$.

By contradiction, assume that there exists a graph $G \in \mathcal{F}$ such that

$$
G \text { is a counterexample to Theorem } 4.2 .2 \text { with }|V(G)|+|E(G)| \text { minimized. }
$$

Thus either

$$
D_{6}(G) \cup D_{7}(G) \cup D_{8}(G)=\varnothing, \text { and } G \notin\left\langle\mathbb{Z}_{3}\right\rangle,
$$

or

$$
\exists u \in D_{6}(G) \cup D_{7}(G) \cup D_{8}(G) \text { such that } G \text { is not } \mathbb{Z}_{3} \text {-extensible from } u \text {. }
$$

For a graph $\Gamma$, let $N(\Gamma)=|V(\Gamma)|+|E(\Gamma)|$. We have the following claims.

Claim 1: If holds, then $G$ is $\mathbb{Z}_{3}$-reduced; if (4.2) holds, then $G-u$ is $\mathbb{Z}_{3}$-reduced.

Assume (4.3) holds. Suppose $G-u$ has a nontrivial subgraph $H$ with $H \in\left\langle\mathbb{Z}_{4}\right\rangle$. Since $G \in \mathcal{F}$, $G / H \in \mathcal{F}$. As $H$ is nontrivial, $N(G / H)<N(G)$. Since $G$ is essentially 6-edge-connected, $G / H$ is also essentially 6 -edge connected. By (4.1), $G / H$ satisfies (i). It follows by Lemma 3.1.4 that $G$ is $A$-extensible from $u$, contrary to (4.1). The proof for the case when (4.2) holds is similar. This proves Claim 1.

By Lemma 1.3.3(ii) and Proposition 1.3.1, any $\mathbb{Z}_{3}$-reduced graph does not have $H_{0}, H_{2}, H_{3}$ and $H_{4}$ as a subgraph. Thus by Claim 1 ,

$G$ (when (4.2) holds) or $G-u$ (when (4.3) holds) does not have $H_{0}, H_{2}, H_{3}$, or $H_{4}$ as a subgraph.

Claim 2: $G$ is cyclically 9-edge-connected.

Suppose that $G$ has a minimal cyclical edge-cut $X$ with $|X|<9$. Let $G_{1}$ and $G_{2}$ be the two components of $G-X$. Since $G$ is essentially 6 -edge connected and since both $G_{1}$ and $G_{2}$ are nontrivial, we have $6 \leq|X| \leq 8$. Let $v_{G_{i}}$ be the new vertex in $G / G_{i}$ onto which $G_{i}$ is contracted, for $i=1,2$. Then

$$
E_{G / G_{1}}\left(v_{G_{1}}\right)=E_{G / G_{2}}\left(v_{G_{2}}\right)=X
$$

Case 1: (4.2) holds. 
Let $b \in Z\left(G, \mathbb{Z}_{3}\right)$. Define $b_{2}: V\left(G / G_{2}\right) \mapsto \mathbb{Z}_{3}$ by

$$
b_{2}(v)= \begin{cases}\sum_{z \in V\left(G_{2}\right)} b(z), & \text { if } v=v_{G_{2}} \\ b(v), & \text { otherwise. }\end{cases}
$$

Then $b_{2} \in Z\left(G / G_{2}, \mathbb{Z}_{3}\right)$ as $b \in Z\left(G, \mathbb{Z}_{3}\right)$. By (4.1) and since $N\left(G / G_{2}\right)<N(G), G / G_{2}$ has a $\left(\mathbb{Z}_{3}, b\right)$-NZF $f_{2}$. Now define $b_{1}: V\left(G / G_{1}\right) \mapsto \mathbb{Z}_{3}$ by

$$
b_{1}(v)= \begin{cases}\sum_{z \in V\left(G_{1}\right)} b(z), & \text { if } v=v_{G_{1}} \\ b(v), & \text { otherwise. }\end{cases}
$$

Then $b_{1} \in Z\left(G / G_{1}, \mathbb{Z}_{3}\right)$ as $b \in Z\left(G, \mathbb{Z}_{3}\right)$. Define $f_{0}^{\prime}=\left.f_{2}\right|_{X}: X \mapsto \mathbb{Z}_{3}^{*}$. Then

$$
\partial f_{0}^{\prime}\left(v_{G_{1}}\right)=-\partial f_{2}\left(v_{G_{2}}\right)=-b_{2}\left(v_{G_{2}}\right)=-\sum_{z \in V\left(G_{2}\right)} b(z)=\sum_{z \in V\left(G_{1}\right)} b(z)=b_{1}\left(v_{G_{1}}\right) .
$$

Since $6 \leq d_{G / G_{1}}\left(v_{G_{1}}\right) \leq 8$, and by $(4.1), G / G_{1}$ is $\mathbb{Z}_{3}$-extensible from $v_{G_{1}}$. Therefore there is a $\left(\mathbb{Z}_{3}, b\right)$-NZF $f_{1}$ of $G / G_{1}$ such that $\left.f_{1}\right|_{X}=f_{0}^{\prime}=\left.f_{2}\right|_{X}$. Then $f=f_{1}+f_{2}-\left.f_{2}\right|_{X}$ is a $\left(\mathbb{Z}_{3}, b\right)$-NZF of $G$, contrary to (4.1).

Case 2: (4.3) holds.

Let $b \in Z\left(G, \mathbb{Z}_{3}\right)$. Assume $u \in V\left(G_{1}\right)$ and $f_{0}: E(u) \mapsto \mathbb{Z}_{3}^{*}$ such that $\partial f_{0}(u)=b(u)$.

Define $b_{2}: V\left(G / G_{2}\right) \mapsto \mathbb{Z}_{3}$ by

$$
b_{2}(v)= \begin{cases}\sum_{z \in V\left(G_{2}\right)} b(z), & \text { if } v=v_{G_{2}}, \\ b(v), & \text { otherwise. }\end{cases}
$$

Then $b_{2} \in Z\left(G / G_{2}, \mathbb{Z}_{3}\right)$ as $b \in Z\left(G, \mathbb{Z}_{3}\right)$. By (4.1) and since $N\left(G / G_{2}\right)<N(G), G / G_{2}$ is $\mathbb{Z}_{3}$-extensible from $u$, and so $G / G_{2}$ has a $\left(\mathbb{Z}_{3}, b\right)$-NZF $f_{2}$ such that $\left.f_{2}\right|_{E(u)}=f_{0}$.

Now define $b_{1}: V\left(G / G_{1}\right) \mapsto \mathbb{Z}_{3}$ by

$$
b_{1}(v)= \begin{cases}\sum_{z \in V\left(G_{1}\right)} b(z), & \text { if } v=v_{G_{1}}, \\ b(v), & \text { otherwise. }\end{cases}
$$

Then $b_{1} \in Z\left(G / G_{1}, \mathbb{Z}_{3}\right)$ as $b \in Z\left(G, \mathbb{Z}_{3}\right)$. For $v_{G_{1}}$, define $f_{0}^{\prime}=\left.f_{2}\right|_{X}: X \mapsto \mathbb{Z}_{3}^{*}$. Then

$$
\partial f_{0}^{\prime}\left(v_{G_{1}}\right)=-\partial f_{2}\left(v_{G_{2}}\right)=-b_{2}\left(v_{G_{2}}\right)=-\sum_{z \in V\left(G_{2}\right)} b(z)=\sum_{z \in V\left(G_{1}\right)} b(z)=b_{1}\left(v_{G_{1}}\right) .
$$

By (4.1), by $N\left(G / G_{1}\right)<N(G)$, and since $6 \leq d_{G / G_{1}}\left(v_{G_{1}}\right) \leq 8, G / G_{1}$ is $\mathbb{Z}_{3}$-extensible from $v_{G_{1}}$. Therefore $G / G_{1}$ has a $\left(\mathbb{Z}_{3}, b_{1}\right)$-NZF $f_{1}$ satisfying $\left.f_{1}\right|_{X}=f_{0}^{\prime}=\left.f_{2}\right|_{X}$. Thus $f=f_{1}+f_{2}-\left.f_{2}\right|_{X}$ is a $\left(\mathbb{Z}_{3}, b\right)$-NZF of $G$ such that $\left.f\right|_{E(u)}=\left.f_{2}\right|_{E(u)}=f_{0}$, contrary to (4.1). This proves Claim 2.

Let $\mathcal{H}=\left\{H_{0}, H_{1}, H_{2}, H_{3}, K_{3}, H_{4}\right\}$. For a graph $G \in \mathcal{F}$, a subgraph $H \subseteq G$ is $\mathcal{H}$-maximal if $H \in\left\{H_{0}, H_{1}, H_{2}, H_{3}, K_{3}, H_{4}\right\}$ and $H$ is not properly contained in another subgraph of $G$ that 
is also a member in $\left\{H_{0}, H_{1}, H_{2}, H_{3}, K_{3}, H_{4}\right\}$. By the definition of $\mathcal{F}$, if $G \in \mathcal{F}$, then every edge must be in an $\mathcal{H}$-maximal subgraph of $G$.

Claim 3: $D_{3}(G) \cup D_{4}(G) \cup D_{5}(G) \neq \varnothing$.

By contradiction, assume that

$$
D_{3}(G) \cup D_{4}(G) \cup D_{5}(G)=\varnothing .
$$

Let $v \in V(G)$ such that if (4.3) holds, then choose $v$ so that $u$ and $v$ are not in the same $\mathcal{H}$-maximal subgraph of $G$. Thus $d_{G}(v) \geq 6$. Since $G \in F$ and by (4.4), $v$ must be in an $\mathcal{H}$-maximal subgraph $H$ of $G$ such that $H \in\left\{K_{3}, K_{4}\right\}$.

Case 1: Suppose $v \in V(H)$ where $H \cong K_{4}$ with $V(H)=\left\{v, x_{1}, x_{2}, x_{3}\right\}$. Let $G_{v}$ be the graph as defined in Lemma 3.1.1, and we shall use the notations in Figures 3.1 and 3.2.

By the definition of $G_{v}, N\left(G_{v}\right)<N(G)$ and $G_{v} \in \mathcal{F}$. If $G_{v}$ is essentially 6-edge-connected, then by (4.1), $G_{v}$ satisfies (i) or (ii). By Lemma 3.1.1, $G$ satisfies (i) or (ii) respectively, contrary to $(4.1)$.

Thus $G_{v}$ has a minimal essential edge cut $X$ with $|X|<6$. Let $G_{1}, G_{2}$ be the two components of $G-X$. Since $G$ is essentially 6-edge-connected, $\left\{x_{1}, x_{2}, x_{3}\right\}$ and $N_{G}(v)-\left\{x_{1}, x_{2}, x_{3}\right\}$ must be in distinct components of $G_{v}-X$. By the assumption that $G \in \mathcal{F}$ and by (4.4), neither $G_{1}$ nor $G_{2}$ is acyclic. It follows that in $G, X \cup\left\{v x_{1}, v x_{2}, v x_{3}\right\}$ is a cyclical edge-cut with at most 8 edges, contrary to Claim 2. This precludes Case 1 of Claim 3.

Case 2: Suppose $v \in V(H)$ where $H \cong K_{3}$ with $V(H)=\left\{v, v_{1}, v_{2}\right\}$. Let $Y=\left\{v v_{1}, v v_{2}\right\}$ and $G_{[v, Y]}$ be the graph defined in Definition 3.1.2. Then $N\left(G_{[v, Y]}\right)<N(G)$. By the choice of $H$, $G_{[v, Y]} \in \mathcal{F}$. If $G_{[v, Y]}$ is essentially 6-edge-connected, then by (4.1), $G_{[v, Y]}$ satisfies (i) or (ii). By Lemma 3.1.3 $G$ satisfies (i) or (ii) respectively, contrary to (4.1).

Thus $G_{[v, Y]}$ must have a minimal essential edge cut $X$ with $|X|<6$. Let $G_{1}, G_{2}$ be the two components of $G_{[v, Y]}-X$. Using the notation in Definition 3.1.2, since $G$ is essentially 6-edge-connected, $v$ and $\left\{v_{1}, v_{2}\right\}$ must be separated by $X$ in $G_{[v, Y]}$. We may assume that $\left\{v_{1}, v_{2}\right\} \subseteq V\left(G_{1}\right)$ and $N_{G}[v]-\left\{v_{1}, v_{2}\right\} \subseteq V\left(G_{2}\right)$. Note that $G_{1}\left[\left\{v_{1}, v_{2}\right\}\right]$ is a 2-circuit, and by (4.4) and since $d_{G}(v) \geq 6, G_{2}$ cannot be acyclic. It follows that $X \cup\left\{v v_{1}, v v_{2}\right\}$ is a cyclical 7-edge-cut of $G$, contrary to Claim 2. This precludes Case 2 of Claim 3, and completes the proof for Claim 3.

Claim 4: $\quad \kappa(G) \geq 2$.

By contradiction, assume that $G$ has two subgraphs $G_{1}, G_{2}$ with $G=G_{1} \cup G_{2}$ and $V\left(G_{1}\right) \cap$ $V\left(G_{2}\right)=\{w\}$. Without loss of generality, if (4.3) holds, we may further assume that $u \in V\left(G_{1}\right)$. By (4.1), $G_{2} \in\left\langle\mathbb{Z}_{4}\right\rangle$, contrary to Claim 1 . This proves Claim 4 . 

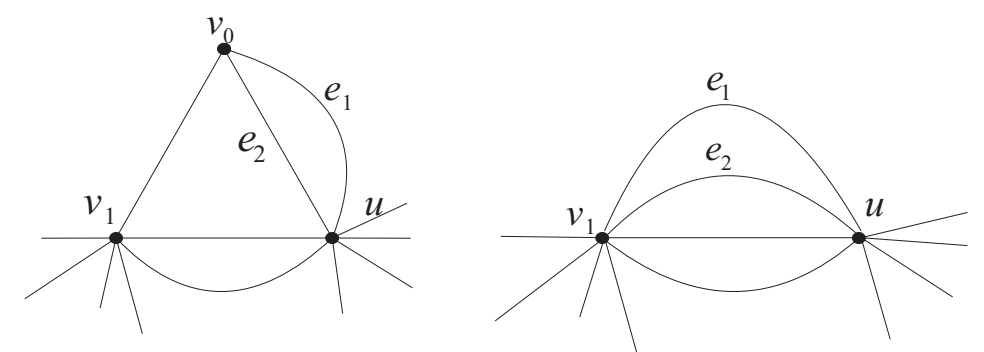

Figure 4.2: Case 1a in the proof of Theorem 2.3.2

By Claim 3, we assume that

$$
D_{3}(G) \cup D_{4}(G) \cup D_{5}(G)=\left\{v_{0}\right\} .
$$

Let $b \in Z\left(G, \mathbb{Z}_{3}\right)$ and $f_{0}: E(u) \mapsto \mathbb{Z}_{3}^{*}$ be such that $\partial f_{0}(u)=b(u)$. Without loss of generality, we assume that all edges in $E_{G}(u)$ are oriented away from $u$.

In the rest of the proof, we shall assume the existence of $u \in D_{6}(G) \cup D_{7}(G) \cup D_{8}(G)$ to prove that $G$ is $\mathbb{Z}_{3}$-extensible from $u$. We shall also show that no matter whether the degree of $v_{0}$ in $G$ is 3,4 or 5 , a contradiction will be obtained. The proof for the case when $D_{6}(G) \cup D_{7}(G) \cup D_{8}(G)=$ $\varnothing$ is similar.

By (4.3), in each of the cases below, we always assume that there exists a $b \in Z\left(G, \mathbb{Z}_{3}\right)$ and an $f_{0}: E_{G}(u) \mapsto \mathbb{Z}_{3}^{*}$ with $\partial f_{0}(u)=b(u)$, such that Theorem 4.2 .2 (i) fails.

Case 1: $v_{0} \in D_{3}(G)$.

Since $v_{0} \in D_{3}(G), G$ has an $\mathcal{H}$-maximal subgraph $H$ with $v_{0} \in V(H)$. By Claim 4 and by $v_{0} \in D_{3}(G), H \in\left\{H_{1}, H_{2}\right\}$. By (4.4), if $H=H_{2}$, then $u$ must be the degree 4 vertex in $H_{2}$.

Case 1a: $H \cong H_{2}$.

Denote $V(H)=\left\{v_{0}, u, v_{1}\right\}$ where $u \in D_{4}(H)$ and $G_{v_{0}}=G /\left\{v_{0} v_{1}\right\}$ (see Figure 4.2). Then $N\left(G_{v_{0}}\right)<N(G)$. Since $G \in \mathcal{F}$ and since $G$ is essentially 6-edge-connected, $G_{v_{0}} \in F$ and $G_{v_{0}}$ is essentially 6-edge connected. By (4.1), $G_{v_{0}}$ satisfies (i).

Define $b^{\prime}: V\left(G_{v_{0}}\right) \mapsto \mathbb{Z}_{3}$ by

$$
b^{\prime}(v)= \begin{cases}b\left(v_{0}\right)+b\left(v_{1}\right), & \text { if } v=v_{1} \\ b(v), & \text { otherwise. }\end{cases}
$$

As $\sum_{v \in V\left(G_{0}\right)} b^{\prime}(v)=\sum_{v \in V(G)} b(v)=0, b^{\prime} \in Z\left(G_{v_{0}}, \mathbb{Z}_{3}\right)$. Since $G_{v_{0}}$ is $\mathbb{Z}_{3}$-extensible from $u$, $\exists f^{\prime} \in F^{*}\left(G_{v_{0}}, \mathbb{Z}_{3}\right)$ such that $\partial f^{\prime}=b^{\prime}$ and $\left.f^{\prime}\right|_{E(u)}=f_{0}$. Assume that the edge $v_{0} v_{1}$ is oriented from $v_{0}$ to $v_{1}$. Define $f: E(G) \mapsto \mathbb{Z}_{3}^{*}$ by

$$
f(e)= \begin{cases}b\left(v_{0}\right)+f^{\prime}\left(e_{1}\right)+f^{\prime}\left(e_{2}\right), & \text { if } e=v_{0} v_{1} \\ f^{\prime}(e), & \text { otherwise. }\end{cases}
$$



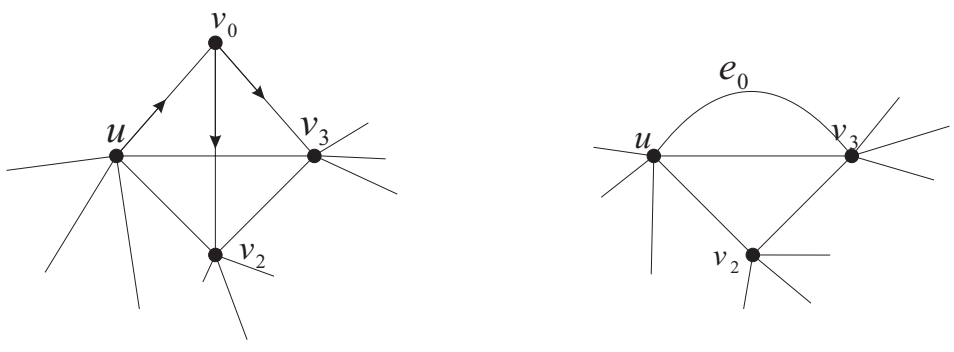

Figure 4.3: Case $1 \mathrm{~b}$ in the proof of Theorem 4.2.2

Then for all $v \in V(G)$,

$$
\partial f(v)= \begin{cases}b\left(v_{0}\right)+f^{\prime}\left(e_{1}\right)+f^{\prime}\left(e_{2}\right)-f^{\prime}\left(e_{1}\right)-f^{\prime}\left(e_{2}\right)=b\left(v_{0}\right) & \text { if } v=v_{0}, \\ \left(b^{\prime}\left(v_{1}\right)+f^{\prime}\left(e_{1}\right)+f^{\prime}\left(e_{2}\right)\right)-\left(b\left(v_{0}\right)+f^{\prime}\left(e_{1}\right)+f^{\prime}\left(e_{2}\right)\right)=b\left(v_{1}\right) & \text { if } v=v_{1}, \\ b^{\prime}(v)=b(v), & \text { otherwise. }\end{cases}
$$

It follows that $\partial f=b$, and $\left.f\right|_{E(u)}=\left.f^{\prime}\right|_{E(u)}=f_{0}$. Therefore $G$ is $\mathbb{Z}_{3}$-extensible from $u$, contrary to (4.1). This completes the proof for Case 1a.

Case 1b: $H=H_{1} \cong K_{4}$ and $u \in V(H)$.

Let $V(H)=\left\{v_{0}, u, v_{2}, v_{3}\right\}$. Define $G_{v_{0}}$ to be the graph obtained from $G-v_{0} v_{2}$ by replacing $u v_{0} v_{3}$ by one edge $e_{0}$ (see Figure 4.3). Then $N\left(G_{v_{0}}\right)<N(G)$.

Suppose that $G_{v_{0}}$ has an essential edge-cut $X$ with $|X|<6$. Since $G$ is essentially 6-edgeconnected, $X$ must separate $v_{0}$ and $v_{2}$. It follows by (4.4) that $X \cup\left\{v_{0} v_{2}\right\}$ is a cyclical edge-cut of $G$ with $\left|X \cup\left\{v_{0} v_{2}\right\}\right| \leq 6$, contrary to Claim 2. Thus $G_{v_{0}}$ is essentially 6-edge-connected and so by $(4.1)$,

$$
G_{v_{0}} \text { is } \mathbb{Z}_{3} \text {-extensible from } u \text {. }
$$

We shall show that $f_{0}$ can be extended to $f \in F^{*}\left(G, \mathbb{Z}_{3}\right)$ to find a contradiction to (4.1).

Case 1b1: $b\left(v_{0}\right)=0$. Define $b^{\prime}: V\left(G_{v_{0}}\right) \mapsto \mathbb{Z}_{3}$ by

$$
b^{\prime}(v)= \begin{cases}b\left(v_{2}\right)-f_{0}\left(u v_{0}\right), & \text { if } v=v_{2}, \\ b\left(v_{3}\right)+f_{0}\left(u v_{0}\right), & \text { if } v=v_{3}, \\ b(v), & \text { otherwise }\end{cases}
$$

Since $\sum_{v \in V\left(G_{v_{0}}\right)} b^{\prime}(v)=\sum_{v \in V(G)} b(v)=0, b^{\prime} \in Z\left(G_{v_{0}}, \mathbb{Z}_{3}\right)$. By (4.6), $\exists f^{\prime} \in F^{*}\left(G_{v_{0}}, \mathbb{Z}_{3}\right)$ such that $\partial f^{\prime}=b^{\prime}$, and $\left.f^{\prime}\right|_{E(u)}=f_{0}$. Assume that $v_{0} v_{2}$ is oriented from $v_{0}$ to $v_{2}$ and $v_{0} v_{3}$ is oriented from $v_{0}$ to $v_{3}$. Define $f: E(G) \mapsto \mathbb{Z}_{3}$ by

$$
f(e)= \begin{cases}f^{\prime}\left(u v_{0}\right), & \text { if } e=v_{0} u, \\ -f^{\prime}\left(u v_{0}\right), & \text { if } e=v_{0} v_{2}, \\ 2 f^{\prime}\left(u v_{0}\right), & \text { if } v=v_{0} v_{3}, \\ f^{\prime}(e), & \text { otherwise. }\end{cases}
$$


Since $f^{\prime} \in F^{*}\left(G_{v_{0}}, \mathbb{Z}_{3}\right), f \in F^{*}\left(G, \mathbb{Z}_{3}\right)$. For each $v \in V(G)$,

$$
\partial f(v)= \begin{cases}2 f^{\prime}\left(u v_{0}\right)-f^{\prime}\left(u v_{0}\right)-f^{\prime}\left(u v_{0}\right)=0=b\left(v_{0}\right), & \text { if } v=v_{0}, \\ \partial f^{\prime}\left(v_{2}\right)-\left(-f^{\prime}\left(u v_{0}\right)\right)=b^{\prime}\left(v_{2}\right)+f^{\prime}\left(u v_{0}\right)=b\left(v_{2}\right), & \text { if } v=v_{2}, \\ b^{\prime}\left(v_{3}\right)+f^{\prime}\left(u v_{0}\right)-2 f^{\prime}\left(u v_{0}\right)=b\left(v_{3}\right), & \text { if } v=v_{3}, \\ \partial f^{\prime}(v)=b^{\prime}(v)=b(v), & \text { otherwise. }\end{cases}
$$

Thus $\partial f=b$ and $\left.f\right|_{E(u)}=\left.f^{\prime}\right|_{E(u)}=f_{0}$. Hence $G$ is $\mathbb{Z}_{3}$-extensible from $u$, contrary to (4.1).

Case 1b2: $\quad b\left(v_{0}\right) \neq 0$.

Define $b^{\prime}: V\left(G_{v_{0}}\right) \mapsto \mathbb{Z}_{3}$ by

$$
b^{\prime}(v)= \begin{cases}b\left(v_{2}\right)+b\left(v_{0}\right), & \text { if } v=v_{2}, \\ b(v), & \text { otherwise. }\end{cases}
$$

Then $b^{\prime} \in Z\left(G_{v_{0}}, \mathbb{Z}_{3}\right)$. By (4.6), $G_{v_{0}}$ has an $f^{\prime}: E\left(G_{v_{0}}\right) \mapsto \mathbb{Z}_{3}^{*}$ such that $\partial f^{\prime}=b^{\prime}$ and $\left.f^{\prime}\right|_{E(u)}=f_{0}$. Assume that $v_{0} v_{2}$ and $v_{0} v_{3}$ are oriented away from $v_{0}$. Define $f: E(G) \mapsto \mathbb{Z}_{3}^{*}$ by

$$
f(e)= \begin{cases}b\left(v_{0}\right), & \text { if } e=v_{0} v_{2}, \\ f^{\prime}\left(v_{0} u\right), & \text { if } e=v_{0} u, v_{0} v_{3}, \\ f^{\prime}(e), & \text { otherwise. }\end{cases}
$$

Since $f^{\prime} \in F^{*}\left(G_{v_{0}}, \mathbb{Z}_{3}\right)$ and since $b\left(v_{0}\right) \neq 0, f \in F^{*}\left(G, \mathbb{Z}_{3}\right)$. For each $v \in V(G)$,

$$
\partial f(v)= \begin{cases}b\left(v_{0}\right)+f^{\prime}\left(v_{0} u\right)-f^{\prime}\left(v_{0} u\right)=b\left(v_{0}\right), & \text { if } v=v_{0}, \\ \partial f^{\prime}\left(v_{2}\right)-b\left(v_{0}\right)=b^{\prime}\left(v_{2}\right)-b\left(v_{0}\right)=b\left(v_{2}\right), & \text { if } v=v_{2}, \\ \partial f^{\prime}(v)=b^{\prime}(v)=b(v), & \text { otherwise. }\end{cases}
$$

Therefore $\partial f=b$ and $\left.f\right|_{E(u)}=\left.f^{\prime}\right|_{E(u)}=f_{0}$. Thus $G$ is $\mathbb{Z}_{3}$-extensible from $u$, contrary to (4.1).

Case 1c: $\quad H=H_{1} \cong K_{4}$ and $u \notin V(H)$.

Let $V(H)=\left\{v_{0}, v_{1}, v_{2}, v_{3}\right\}$. Then $d_{G}\left(v_{i}\right) \geq 6$ for $i=1,2,3$. Let $G_{v_{1}}$ be the graph obtained from $G$ by first splitting the vertex $v_{1} \in V(G)$ into $v_{1}, v_{1}^{\prime}$ (where $v_{1}^{\prime}$ is adjacent to $v_{0}, v_{2}, v_{3}$ ), deleting the edge $v_{1}^{\prime} v_{2}$, and then contracting $v_{1}^{\prime} v_{3}$ (see Figure 4.4). As before, if $G_{v_{1}}$ has an essential edge cut $X$ with $|X|<6$, then $X$ must separate $v_{1}$ and $\left\{v_{0}, v_{2}, v_{3}\right\}$, and so $X \cup$ $\left\{v_{1} v_{0}, v_{1} v_{2}, v_{1} v_{3}\right\}$ is a cyclical edge cut of $G$. It follows by Claim 2 that $G_{v_{1}}$ is essentially 6-edge-connected.

Let $L^{\prime}=G_{v_{1}}\left[\left\{v_{0}, v_{2}, v_{3}\right\}\right]$. As $L^{\prime}$ is the three vertex graph with four edges, $L^{\prime} \in\left\langle\mathbb{Z}_{4}\right\rangle$. Let $G^{\prime}=G_{v_{1}} / L^{\prime}$ with a new vertex $v_{L^{\prime}}$. Define $b_{1}: V\left(G_{v_{1}}\right) \mapsto \mathbb{Z}_{3}$ such that $b_{1}(v)=b(v)$, for all $v \in V\left(G_{v_{1}}\right)$. As $b \in Z\left(G, \mathbb{Z}_{3}\right), b_{1} \in Z\left(G_{v_{1}}, \mathbb{Z}_{3}\right)$. Define $b^{\prime}: V\left(G^{\prime}\right) \mapsto \mathbb{Z}_{3}$ to be

$$
b^{\prime}(v)= \begin{cases}b_{1}\left(v_{0}\right)+b_{1}\left(v_{2}\right)+b_{1}\left(v_{3}\right), & \text { if } v=v_{L^{\prime}}, \\ b_{1}(v), & \text { otherwise. }\end{cases}
$$




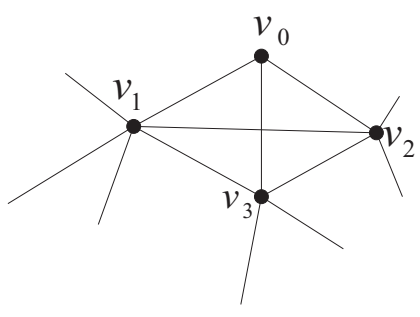

$G$

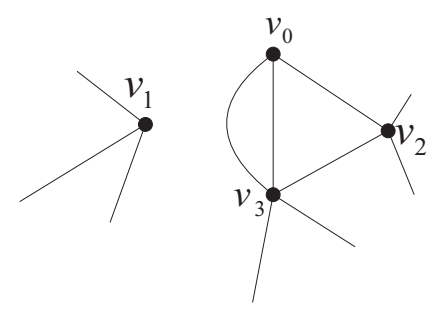

$G_{v_{1}}$

Figure 4.4: Case 1c in the proof of Theorem 2.3.2
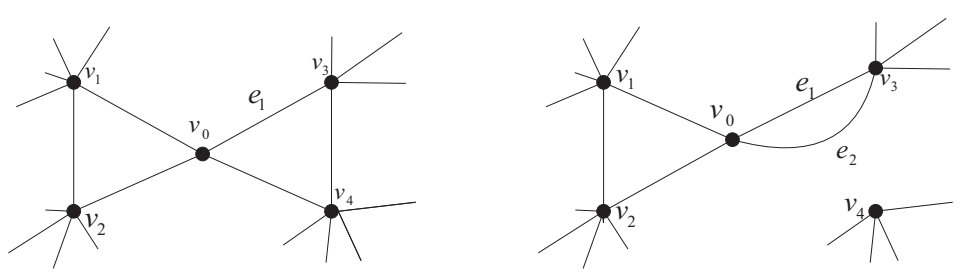

Figure 4.5: Case 2a

Then as $b_{1} \in Z\left(G_{v_{1}}, \mathbb{Z}_{3}\right), b^{\prime} \in Z\left(G^{\prime}, \mathbb{Z}_{3}\right)$.

As $G_{v_{1}}$ is essentially 6-edge-connected, so is $G^{\prime}$. By (4.1), $G^{\prime}$ satisfies (i). For any $\left(\mathbb{Z}_{3}, b^{\prime}\right)$-NZF $f^{\prime}$ of $G^{\prime}$, by Lemma 3.1.4, $f^{\prime}$ can be extended to a $\left(\mathbb{Z}_{3}, b_{1}\right)$-NZF $f_{1}$ of $G_{v_{1}}$, and by Lemma 3.1.3, $f_{1}$ can be extended to a $\left(\mathbb{Z}_{3}, b\right)$-NZF $f$ of $G$. Therefore $G$ satisfies (i), a contrary to (4.1).

Case 2: $v_{0} \in D_{4}(G)$.

Since $G \in \mathcal{F}$, either $G$ has two $\mathcal{H}$-maximal subgraphs $H^{\prime}, H^{\prime \prime}$ isomorphic to $K_{3}$, with $v_{0} \in$ $V\left(H^{\prime}\right) \cap V\left(H^{\prime \prime}\right)$, or $G$ has an $\mathcal{H}$-maximal subgraph $H \cong H_{2}$ with $v_{0} \in V(H)$, as by Claim 4 , $H \cong H_{0}$ is impossible.

Case 2a: Suppose $v_{0} \in V\left(H^{\prime}\right) \cap V\left(H^{\prime \prime}\right)$ for two maximal subgraph $H^{\prime} \cong H^{\prime \prime} \cong K_{3}$ (see Figure $4.5)$.

Let $N_{G}\left(v_{0}\right)=\left\{v_{1}, v_{2}, v_{3}, v_{4}\right\}$. Without loss of generality, we may assume that $V\left(H^{\prime \prime}\right)=$ $\left\{v_{0}, v_{3}, v_{4}\right\}$ and $u \notin V\left(H^{\prime \prime}\right)$. Let $Y=\left\{v_{4} v_{0}, v_{4} v_{3}\right\}$ and define $G_{\left[v_{4}, Y\right]}$ as in Definition 3.1.2. Denote the two parallel edges joining $v_{0}$ and $v_{3}$ by $e_{1}, e_{2}$. Let $G_{v_{4}}=G_{\left[v_{4}, Y\right]} /\left\{e_{1}, e_{2}\right\}$. Then $N\left(G_{v_{4}}\right)<N(G)$. As before, if $G_{v_{4}}$ has an essential edge cut $X$ with $|X|<6$, then $X$ must separate $v_{4}$ and $v_{0}$ in $G_{v_{4}}$, and so $X \cup\left\{v_{4} v_{0}, v_{4} v_{3}\right\}$ is a cyclical edge cut of $G$. It follows by Claim 2 that $G_{v_{4}}$ is essentially 6-edge-connected. By (4.1), $G_{v_{4}}$ satisfies Theorem 4.2.2(i). By Lemma 3.1.3, $G$ also satisfies Theorem 4.2.2(i), contrary to (4.1). 


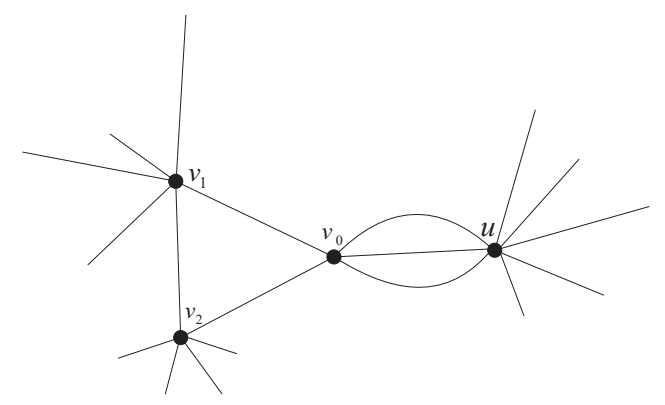

Figure 4.6: $\left(H^{\prime}, H^{\prime \prime}\right)=\left(K_{3}, H_{3}\right)$ in Case 3

Case 2b: Suppose $v_{0}$ is contained in a subgraph $H \cong H_{2}$.

Since $G \in \mathcal{F}, d_{G}\left(v_{0}\right)=d_{H}\left(v_{0}\right)=4, G$ must have a 2-circuit which does not contain $u$ as a vertex, contrary to (4.4). This precludes Case 2 .

Case 3: $v_{0} \in D_{5}(G)$

Since $G \in \mathcal{F}$, by the definition of $\mathcal{F}, G$ must have two $\mathcal{H}$-maximal subgraphs $H^{\prime}, H^{\prime \prime}$ such that $H^{\prime} \in\left\{K_{3}, H_{4}\right\}$ and $H^{\prime \prime} \in\left\{H_{1}, H_{2}, H_{3}\right\}$ with $v_{0} \in V\left(H^{\prime}\right) \cap D_{3}\left(H^{\prime \prime}\right)$. By (4.4), $H^{\prime}$ and $H^{\prime \prime}$ cannot both have multiple edges, and so

$$
\left(H^{\prime}, H^{\prime \prime}\right) \in\left\{\left(K_{3}, H_{1}\right),\left(H_{4}, H_{1}\right),\left(K_{3}, H_{2}\right),\left(K_{3}, H_{3}\right)\right\} .
$$

If $\left(H^{\prime}, H^{\prime \prime}\right)=\left(K_{3}, H_{3}\right)$, (see Figure 4.6), then let $V\left(K_{3}\right)=\left\{v_{0}, v_{1}, v_{2}\right\}$ and $V\left(H_{3}\right)=\left\{v_{0}, v_{3}\right\}$. By (4.4), $u=v_{3}$. Let $V_{1}=\left\{v_{0}, u\right\}, V_{2}=V(G)-V_{1}$, and $W$ be the set of edges with one end in $V_{1}$ and the other in $V_{2}$. Since $d_{G}(u) \leq 8,|W| \leq 2+d_{G}(u)-3<8$, and so $X$ is a cyclical edge cut of $G$ with at most seven edges, contrary to Claim 2.

Assume that $\left(H^{\prime}, H^{\prime \prime}\right)=\left(K_{3}, H_{1}\right)$. Let $V\left(K_{3}\right)=\left\{v_{0}, v_{1}, v_{2}\right\}$, and define $Y=\left\{v_{0} v_{1}, v_{0} v_{2}\right\}$. Define $G_{\left[v_{0}, Y\right]}$ as in Definition 3.1.2. Then $N\left(G_{\left[v_{0}, Y\right]}\right)<N(G)$. If $G_{\left[v_{0}, Y\right]}$ has an essential edge cut $X$ with $|X|<6$, then $X$ must separate $V\left(K_{3}\right)-\left\{v_{0}\right\}$ and $V\left(H_{1}\right)-\left\{v_{0}\right\}$ in $G_{\left[v_{0}, Y\right]}$, and so $X \cup\left\{v_{0} v_{1}, v_{0} v_{2}\right\}$ is a cyclical edge cut of $G$. It follows by Claim 2 that $G_{\left[v_{0}, Y\right]}$ is essentially 6-edgeconnected. By (4.1), $G_{\left[v_{0}, Y\right]}$ satisfies (i). By Lemma 3.1.4 $G$ also satisfies (i) of Theorem 4.2.2, contrary to (4.1).

Next, we assume that $\left(H^{\prime}, H^{\prime \prime}\right)=\left(H_{4}, H_{1}\right)$. Then by (4.4), we denote $V\left(H_{1}\right)=\left\{v_{0}, z_{1}, z_{2}, z_{3}\right\}$ and $V\left(H_{4}\right)=\left\{v_{0}, u\right\}$ (see Figure 4.7). Let $G_{z_{1}}$ be the graph obtained from $G$ by first splitting the vertex $z_{1} \in V(G)$ into $z_{1}, z_{1}^{\prime}$ (where $z_{1}^{\prime}$ is adjacent to $v_{0}, z_{2}, z_{3}$ ), deleting the edge $z_{1}^{\prime} z_{2}$, and the contracting $z_{1}^{\prime} z_{3}$. If $G_{z_{1}}$ has an essential edge cut $X$ with $|X|<6$, then $X$ must separate $z_{1}$ and $v_{0}, z_{2}, z_{3}$ in $G_{z_{1}}$, and so $X \cup\left\{z_{1} v_{0}, z_{1} z_{2}, z_{1} z_{3}\right\}$ is a cyclical edge cut of $G$. It follows by Claim 2 that $G_{z_{1}}$ is essentially 6-edge-connected. Let $L^{\prime}=G_{z_{1}}\left[\left\{v_{0}, z_{2}, z_{3}\right\}\right]$. As $L^{\prime}$ is the 3 vertex 


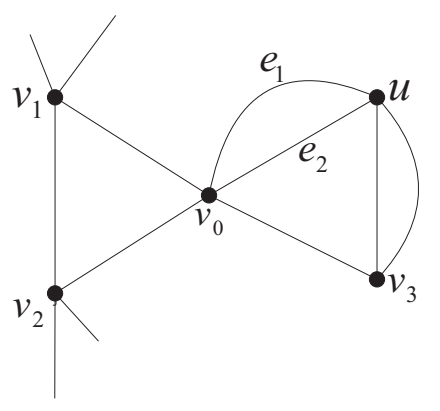

$G$

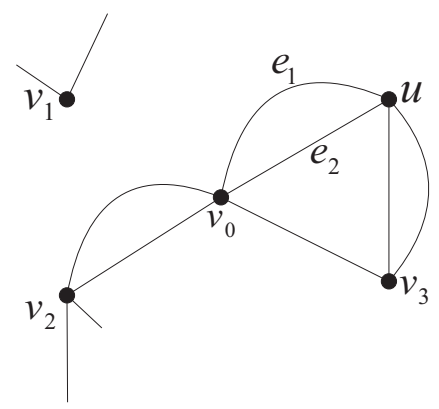

$G_{\left[v_{1}, Y\right]}$

Figure 4.7: $\left(H^{\prime}, H^{\prime \prime}\right)=\left(H_{4}, H_{1}\right)$ in Case 3

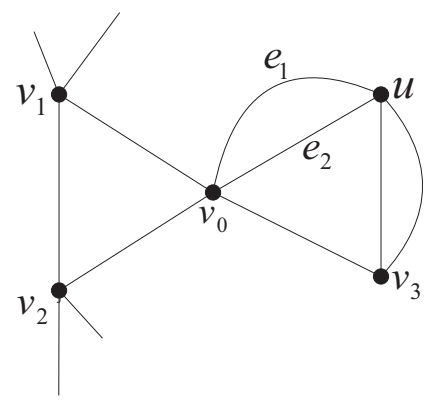

$G$

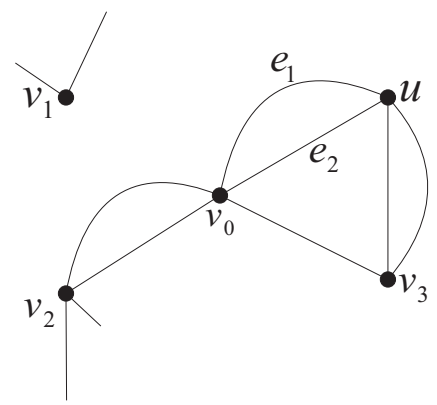

$G_{\left[v_{1}, Y\right]}$

Figure 4.8: $\left(H^{\prime}, H^{\prime \prime}\right)=\left(K_{3}, H_{2}\right)$ in Case 3

graph with 4 edges, $L^{\prime} \in\left\langle\mathbb{Z}_{4}\right\rangle$. Let $G^{\prime}=G_{z_{1}} / L^{\prime}$. As $G_{z_{1}}$ is essentially 6-edge-connected, so is $G^{\prime}$. By (4.1), $G^{\prime}$ satisfies (i). By Lemma 3.1.4, $G_{z_{1}}$ satisfies (i). It follows by Lemma 3.1.1 that $G$ satisfies (i), a contrary to (4.1).

Therefore, we must have $\left(H^{\prime}, H^{\prime \prime}\right)=\left(K_{3}, H_{2}\right)$. Since $v_{0} \in V\left(H^{\prime}\right) \cap V\left(H^{\prime \prime}\right)$, we may assume that $V\left(H^{\prime}\right)=\left\{v_{0}, v_{1}, v_{2}\right\}$. By (4.4), $u$ must be the only vertex of degree four in $H^{\prime \prime}$. Let $e_{1}$ and $e_{2}$ denote the two parallel edges joining $v_{0}$ and $u$ (see Figure 4.8).

Note that $d_{G}\left(v_{1}\right) \geq 6$. Let $Y=\left\{v_{1} v_{0}, v_{1} v_{2}\right\}$. Define $G_{\left[v_{1}, Y\right]}$ as in Definition 3.1.2. By the definition of $\mathcal{F}, G_{\left[v_{1}, Y\right]} \in \mathcal{F}$. If $G_{\left[v_{1}, Y\right]}$ has an essential edge cut $X$ with $|X|<6$, then $X$ must separate $v_{1}$ and $v_{0}$ (see Figure 4.7) in $G_{\left[v_{1}, Y\right]}$, and so $X \cup\left\{v_{1} v_{0}, v_{1} v_{2}\right\}$ is a cyclical edge cut of $G$. It follows by Claim 2 that $G_{\left[v_{1}, Y\right]}$ is essentially 6-edge-connected.

Let $L^{\prime}=G_{\left[v_{1}, Y\right]}\left[\left\{v_{0}, v_{2}\right\}\right]$, which is a 2 -circuit, and so $L^{\prime} \in\left\langle\mathbb{Z}_{4}\right\rangle$. Let $G^{\prime}=G_{\left[v_{1}, Y\right]} / L^{\prime}$. As 
$G_{\left[v_{1}, Y\right]}$ is essentially 6-edge-connected, so is $G^{\prime}$. By (4.1), $G^{\prime}$ satisfies (i). By Lemma 3.1.4, $G_{\left[v_{1}, Y\right]}$ satisfies (i). It follows by Lemma 3.1.3 that $G$ satisfies (i), contrary to (4.1). This completes the proof for Case 3.

As all the cases lead to contradictions, the theorem is established. 


\section{Chapter 5}

\section{An Upper Bound on Group Connectivity}

\subsection{Introduction}

Graphs in this chapter are finite and connected, with parallel edges permitted.

It is known that if $G$ is a loopless plane graph without cut edges and with geometric dual $G^{*}$, then $G$ has a mapping $f: E(G) \mapsto A^{*}$ with $\partial f=0$ if and only if $\chi\left(G^{*}\right)=|A|$ (Tutte [36]). In addition, $\Lambda_{g}(G)=\chi_{g}\left(G^{*}\right)$ ([16], also see Theorem 3.6 of [7]).

Brooks' Theorem states that equality in the trivial bound $\chi(G) \leq \Delta(G)+1$ hold among connected graphs if and only if $G$ is an odd circuit or a complete graph. The group coloring analogue (Theorem 4.2 of [30]) implies that if $G$ is a 2-edge-connected plane graph, then $\Lambda_{g}(G) \leq$ $\Delta\left(G^{*}\right)+1$. The fact that edges incident with a vertex in $G^{*}$ induce a circuit in $G$ motivates us to consider the problem of using certain circuit lengths of $G$ to describe best possible upper bounds for $\Lambda_{g}(G)$ for general 2-edge-connected graphs that may not be planar. An objective of this paper is to seek best possible upper bounds on $\Lambda_{g}(G)$ with such a feature.

Let $P$ denote a path in $G$, and let $\beta_{G}(P)$ be the minimum length of a circuit containing $P$. For a positive integer $i$, let $\beta_{i}(G)$ be the maximum of $\beta_{G}(P)$ over paths of length $i$ in $G$. By this definition, we have

$$
\operatorname{girth}(G) \leq \beta_{1}(G) \leq \beta_{2}(G) \leq \cdots \leq \beta_{i}(G) \leq \beta_{i+1}(G) \leq \cdots \leq \beta_{c(G)}(G)=c(G)
$$

Let $H_{1}$ and $H_{2}$ be two subgraphs of a graph $G$. We say that $G$ is a parallel connection of $H_{1}$ and $H_{2}$, if $E\left(H_{1}\right) \cup E\left(H_{2}\right)=E(G),\left|V\left(H_{1}\right) \cap V\left(H_{2}\right)\right|=2$ and $\left|E\left(H_{1}\right) \cap E\left(H_{2}\right)\right|=1$.

In Section 2, we shall show that for any positive integer $i$ with $i \geq 1$,

$$
\Lambda_{g}(G) \leq \beta_{i}(G)+1
$$


Determining exactly when the equality $\Lambda_{g}(G)=\beta_{1}(G)+1$ holds seems to be difficult. When $\beta_{1}(G)=3$, Fan et al. [9] solved a special case of this problem by showing that if in $G$ every pair of edges are connected by a sequence of mutually intersecting circuits of length at most 3 , then $\Lambda_{g}(G)=\beta_{1}(G)+1$ if and only if $G$ can be constructed from odd wheels and $K_{3}$ by a finite number of parallel connections. $\mathrm{Xu}$ and Zhang [38] conjectured a weaker version of Tutte's 3-flow conjecture (see [15] and [39]): if $G$ is 4-edge-connected and $\beta_{1}(G)=3$, then there exists $f: E(G) \mapsto \mathbb{Z}_{3}^{*}$ with $\partial f=0$. It was further conjectured by DeVos ([8], [28]) that every 4-edge-connected graph $G$ with $\beta_{1}(G)=3$ satisfies $\Lambda_{g}(G) \leq 3$. This stronger conjecture was disproved in [28]. As of today, it is not known (see [28]) whether every 5-edge-connected graph $G$ with $\beta_{1}(G)=3$ satisfies $\Lambda_{g}(G) \leq 3$. See a recent survey [25] for more in the literature.

The main purpose of this chapter is to prove the inequality (5.2) and to determine for $i=2$ the graphs such that equality holds in (5.2). To describe the main result of this paper, we need to introduce some notation.

Theorem 5.1.1 If $G$ is a 2-connected graph, then

$$
\Lambda_{g}(G) \leq \beta_{2}(G)+1
$$

where equality holds in (5.3) if and only if $G \in\left\{C_{k}: k \geq 2\right\} \cup\left\{K_{2, t}^{1 / m}: m \geq 1, t \geq 3\right\} \cup\left\{K_{4}^{1 / k}: k \geq\right.$ $1\}$.

Corollary 5.1.2 If $G$ is a 2-connected graph, then

$$
\Lambda_{g}(G) \leq c(G)+1
$$

where equality holds if and only if either $c(G)$ is odd and $G$ is an odd circuit, or $c(G)$ is even and $G$ is isomorphic to a $K_{2, t}^{1 /(c(G) / 2)}$, for some $t$.

Corollary 5.1.3 If $G$ is a 2-edge-connected graph, then (5.4) holds, with equality if and only if each of the following holds:

(i) $G$ has at least one block $B$ such that either $c(G)$ is odd and $B$ is an odd circuit of length $c(G)$, or $c(G)$ is even and $B$ is isomorphic to a $K_{2, t}^{1 /(c(G) / 2)}$, for some $t$.

(ii) Every block $H$ of $G$ is either a subgraph with $\Lambda_{g}(H) \leq c(G)$, or $c(G)$ is odd and $H$ is a circuit of length $c(G)$, or $c(G)$ is even and $H$ is isomorphic to a $K_{2, t}^{1 /(c(G) / 2)}$, for some integer $t \geq 2$.

Jaeger et al. [16] showed that if $G$ is 3-edge-connected, then $\Lambda_{g}(G) \leq 6$, which extends Seymour's famous 6-flow theorem from [33]. Thus it is clear that when $\beta_{2}(G) \geq 6$, all the extremal graphs in Theorem 5.1.1 will have 2-edge cuts. In Section 2, we investigate some preliminary properties of $\beta_{2}(G)$, which lead to a proof for (5.3), and present the extremal graphs 
in Theorem 5.1.1, as well as the proofs for Corollaries 5.1.2 and 5.1.3. In Section 3, we complete the characterization of the extremal graphs in Theorem 5.1.1. We make some remarks on the applications of Theorem 5.1.1 in the last section.

Let $G$ be a graph and $H$ be a subgraph of $G$. Following Seymour [33], we define the $k$-closure of $H$ in $G$, denoted $c l_{k}(H)$, to be $H \cup C^{1} \cup C^{2} \cup \cdots$, where $C^{1}, C^{2}, \ldots$ are circuits of $G$ such that $\left|E\left(C^{i}\right)-\left(E(H) \cup \cup_{j=1}^{i-1} E\left(C^{j}\right)\right)\right| \leq k$.

Corollary 5.1.4 Let $G$ be a graph and $H$ be a subgraph of $G$ with $c l_{k}(H)=G$. Let $A$ be an Abelian group with $|A| \geq k+1$. If $H$ is $A$-connected, then $G$ is also A-connected. In particular, if $\Lambda_{g}(H) \leq k+1$ and if $c l_{k}(H)=G$, then $\Lambda_{g}(G) \leq k+1$.

Proof: Suppose that $H \cup C^{1} \cup \ldots \cup C^{m}=G$. We argue by induction on $m$ to show that for any $A$ with $|A| \geq \Lambda_{g}(H), G \in\langle A\rangle$. Since $|A| \geq \Lambda_{g}(H)$, this holds if $m=0$. Now assume that $m \geq 1$. Let $H^{\prime}=H \cup C^{1} \cup \ldots \cup C^{m-1}$. By the induction hypothesis, $H^{\prime} \in\langle A\rangle$. By the definition of $k$-closure, every circuit of $G / H^{\prime}=C^{m} /\left(C^{m} \cap H^{\prime}\right)$ has length at most $k$, where $k<|A|$. By Lemmas 1.3.3 (ii) and 1.3.7, $G / H^{\prime} \in\langle A\rangle$. It follows by Proposition 1.3.1(C3) that $G \in\langle A\rangle$.

Lemma 5.1.5 For any graph $G$ with $\kappa^{\prime}(G) \geq 2$,

$$
\Lambda_{g}(G) \leq \max \left\{\operatorname{girth}(G)+1, \beta_{1}(G)\right\} \leq \max \left\{\operatorname{girth}(G)+1, \beta_{2}(G)\right\} \leq \beta_{2}(G)+1 .
$$

Proof: By (5.1), girth $(G) \leq \beta_{1}(G)$. If $\beta_{1}(G)=\operatorname{girth}(G)$, then for every edge $e \in E(G)$, $G$ has a circuit $C_{e}$ with length $\operatorname{girth}(G)$ and with $e \in E\left(C_{e}\right)$. By Lemma 1.3.3 (ii), for any Abelian group $A$ with $|A| \geq \operatorname{girth}(G)+1, C_{e} \in\langle A\rangle$. By Lemma 1.3.7, $G \in\langle A\rangle$, and so $\Lambda_{g}(G) \leq \operatorname{girth}(G)+1=\beta_{1}(G)+1$. Hence we may assume $\beta_{1}(G)>\operatorname{girth}(G)$. Let $C^{0}$ be a circuit in $G$ with $\left|E\left(C^{0}\right)\right|=\operatorname{girth}(G)$. By Lemma 1.3.3 (ii), $\Lambda_{g}\left(C^{0}\right)=\operatorname{girth}(G)+1 \leq \beta_{1}(G)$. By the definition of $\beta_{1}(G)$ and by $\kappa^{\prime}(G) \geq 2, c_{\beta_{1}(G)-1}\left(C^{0}\right)=G$. By Corollary 5.1.4, $\Lambda_{g}(G) \leq \beta_{1}(G)$. Hence we proved the first inequality of (5.5). The second inequality of (5.5) follows from (5.1).

Thus (5.2) and (5.3), now follow from (5.1) and (5.5). Let $\mathcal{E}$ denote the set of all 2-connected graphs satisfying equality in (5.3), and define

$$
\mathcal{E}_{k}=\left\{G \in \mathcal{E}: \operatorname{girth}(G)=\beta_{2}(G)=k\right\} .
$$

By Lemma 1.3.3 (ii), $C_{k} \in \mathcal{E}_{k}$. We next show that two other classes of graphs are also in $\mathcal{E}_{k}$.

Lemma 5.1.6 Let $t \geq 2$ and $m \geq 1$ be integers. If $G \cong K_{2, t}^{1 / m}$, then $\Lambda_{g}(G)=\beta_{2}(G)+1$.

Proof: The lemma holds trivially for $m=1$, and so we assume that $m \geq 2$. Let the two (nonadjacent) vertices of degree $t$ in $G$ be $w_{1}$ and $w_{2}$; and let the vertices of degree 2 in $V(G)-$ $\left\{w_{1}, w_{2}\right\}$ be $v_{j}^{i}$, for $1 \leq j \leq m-1$ and $1 \leq i \leq t$, such that for each $i$ with $1 \leq i \leq t$, 


$$
w_{1}, v_{1}^{i}, v_{2}^{i}, \cdots, v_{m-1}^{i}, w_{2}
$$

is a directed path under a fixed orientation $D$ of $G$. Note that in this case, $\beta_{2}(G)=2 m$, and by $(5.5), \Lambda_{g}(G) \leq 2 m+1$. To prove that $\Lambda_{g}(G)=2 m+1$, it suffices to show that $G$ is not $\left\langle\mathbb{Z}_{4}\right\rangle_{2 m}$-connected.

We shall apply the equivalence between Theorem 1.3.2(i) and (iii) to prove that $G$ is not $\left\langle\mathbb{Z}_{4}\right\rangle_{2 m}$-connected. Let $A=\left\langle\mathbb{Z}_{4}\right\rangle_{2 m}$, and let $A_{e}=\{\overline{2}, \overline{4}, \cdots, \overline{2 m-2}\} \subset\left\langle\mathbb{Z}_{4}\right\rangle_{2 m}$. We shall assume that $G$ is $A$-connected to show that either of the the following two cases will lead to a contradiction.

Case 1: $t=2 k$. Choose $b: V(G) \mapsto A$ to be the mapping given by

$$
b(z)= \begin{cases}\overline{0} & \text { if } z \notin\left\{w_{1}, w_{2}\right\} \\ \overline{1} & \text { if } z=w_{1} \\ -\overline{1} & \text { if } z=w_{2}\end{cases}
$$

Note that $b \in Z(G, A)$. Choose $\bar{f}: E(G) \mapsto A$ by, for each $i$ with $1 \leq i \leq t$,

$$
\bar{f}(e)= \begin{cases}\overline{1} & \text { if } e=\left(w_{1}, v_{1}^{i}\right) \\ \overline{3} & \text { if } e=\left(v_{1}^{i}, v_{2}^{i}\right) \\ \vdots & \vdots \\ \overline{2 j+1} & \text { if } e=\left(v_{j}^{i}, v_{j+1}^{i}\right) \\ \vdots & \vdots \\ \overline{2 m-1} & \text { if } e=\left(v_{m-1}^{i}, w_{2}\right) .\end{cases}
$$

Since $G$ is $A$-connected, by Theorem 1.3.2(iii) there must be a function $f \in F(G, A)$ such that $\partial f=b$ and such that $f(e) \neq \bar{f}(e)$ for $e \in E(G)$. For each $i$ with $1 \leq i \leq t$, let $x_{i}=$ $f\left(w_{1}, v_{1}^{i}\right)$. Since $b(z)=0$ for $z=v_{j}^{i}$, and since the path $w_{1}, v_{1}^{i}, v_{2}^{i}, \ldots, v_{m-1}^{i}, w_{2}$ is a directed path, $f\left(v_{j}^{i}, v_{j+1}^{i}\right)=x_{i}$ for $1 \leq j \leq m-2$ and $f\left(v_{m-1}^{i}, w_{2}\right)=x_{i}$. By the choice of $\bar{f}$, since $f-\bar{f} \in F^{*}(G, A)$, we must have $x_{i} \in A_{e}$. It follows by $\partial f\left(w_{1}\right)=b\left(w_{1}\right)$ that $1 \equiv \sum_{i=1}^{t} x_{i}$ (mod $2 m)$. This implies that the sum of certain even numbers can be equal to an odd number, leading to a contradiction.

Case 2: $t=2 k+1$. Choose $b: V(G) \mapsto A$ to be the mapping given by $b(z)=0$, for all $z \in V(G)$, 
so $b \in Z(G, A)$. Choose $\bar{f}: E(G) \mapsto A$ by defining, for each $i$ with $2 \leq i \leq t$,

$$
\bar{f}(e)= \begin{cases}\overline{1} & \text { if } e=\left(w_{1}, v_{1}^{i}\right) \\ \overline{3} & \text { if } e=\left(v_{1}^{i}, v_{2}^{i}\right) \\ \vdots & \vdots \\ \overline{2 j+1} & \text { if } e=\left(v_{j}^{i}, v_{j+1}^{i}\right) \\ \vdots & \vdots \\ \overline{2 m-1} & \text { if } e=\left(v_{m-1}^{i}, w_{2}\right) .\end{cases}
$$

and,

$$
\bar{f}(e)= \begin{cases}\overline{0} & \text { if } e=\left(w_{1}, v_{1}^{1}\right) \\ \overline{2} & \text { if } e=\left(v_{1}^{1}, v_{2}^{1}\right) \\ \vdots & \vdots \\ \overline{2 j} & \text { if } e=\left(v_{j}^{1}, v_{j+1}^{1}\right) \\ \vdots & \vdots \\ \overline{2 m-2} & \text { if } e=\left(v_{m-1}^{1}, w_{2}\right) .\end{cases}
$$

Since $G$ is $A$-connected, by Theorem 1.3.2(iii) there must be a function $f \in F(G, A)$ such that $\partial f=b$ and such that $f(e) \neq \bar{f}(e)$ for $e \in E(G)$. For each $i$ with $1 \leq i \leq t$, let $x_{i}=$ $f\left(w_{1}, v_{1}^{i}\right)$. Since $b(z)=0$ for $z=v_{j}^{i}$, and since the path $w_{1}, v_{1}^{i}, v_{2}^{i}, \ldots, v_{m-1}^{i}, w_{2}$ is a directed path, $f\left(v_{j}^{i}, v_{j+1}^{i}\right)=x_{i}$ for $1 \leq j \leq m-2$ and $f\left(v_{m-1}^{i}, w_{2}\right)=x_{i}$. By the choice of $\bar{f}$, since $f-\bar{f} \in F^{*}(G, A)$, we must have $x_{i} \in A_{e}$ for $i>1$, and $x_{1} \in A-A_{e}$. It follows by $\partial f\left(w_{1}\right)=b\left(w_{1}\right)$ that $0 \equiv \sum_{i=1}^{t} x_{i}(\bmod 2 m)$. This implies that the sum of certain even numbers plus one odd number can be equal to an even number, leading to a contradiction.

These contradictions establish the validity of the lemma.

Lemma 5.1.7 If $k$ is a positive integer, then $\Lambda_{g}\left(K_{4}^{1 / k}\right)=\beta_{2}\left(K_{4}^{1 / k}\right)+1=3 k+1$.

Proof: By the definition of $K_{4}^{1 / k}, \beta_{2}\left(K_{4}^{1 / k}\right)=3 k$. By (5.5), it suffices to prove that $K_{4}^{1 / k} \notin$ $\left\langle\mathbb{Z}_{3} \times\left\langle\mathbb{Z}_{4}\right\rangle_{k}\right\rangle$. Denote the four vertices of degree 3 in $K_{4}^{1 / k}$ by $v_{1}, v_{2}, v_{3}, v_{4}$ and orient the edges as shown in Figure 5.1.

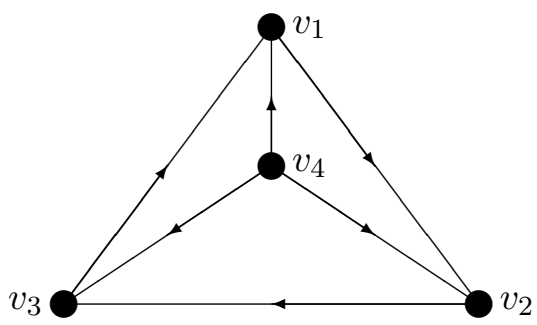


Figure 5.1 Oriented $K_{4}^{1 / k}$, each line representing a path of $k$ edges.

Let $P^{\left(v_{i}, v_{j}\right)}$ denote the directed $\left(v_{i}, v_{j}\right)$-path whose internal vertices have degree 2 , and label these paths by

$$
P_{1}=P^{\left(v_{1}, v_{4}\right)}, P_{2}=P^{\left(v_{2}, v_{4}\right)}, P_{3}=P^{\left(v_{3}, v_{4}\right)}, P_{4}=P^{\left(v_{2}, v_{3}\right)}, P_{5}=P^{\left(v_{3}, v_{1}\right)} \text {, and } P_{6}=P^{\left(v_{1}, v_{2}\right)} .
$$

Let $\bar{f} \in F\left(K_{4}^{1 / k}, \mathbb{Z}_{3} \times\left\langle\mathbb{Z}_{4}\right\rangle_{k}\right)$ be a function such that for each $P^{\left(v_{i}, v_{j}\right)}, \bar{f}: E\left(P^{\left(v_{i}, v_{j}\right)}\right) \rightarrow\{\overline{0}\} \times\left\langle\mathbb{Z}_{4}\right\rangle_{k}$ is surjective. We argue by contradiction and assume that there exists an $A$-flow $f$ such that $f(e) \neq \bar{f}(e)$ for any $e \in E\left(K_{4}^{1 / k}\right)$. Since $\partial f=0, f$ must have the same value on every edge in $E\left(P^{\left(v_{i}, v_{j}\right)}\right)$. For $1 \leq j \leq 6$, let $\left(x_{j}, y_{j}\right)$ denote the common value of $f$ on the edges of $P_{j}$, where $x_{i} \in \mathbb{Z}_{3}, y_{i} \in \mathbb{Z}_{k}$. Then we have $x_{i} \neq \overline{0}$ for $1 \leq i \leq 6$ and $x_{1}+x_{2}+x_{3}=\overline{0}$. Hence $x_{1}=x_{2}=$ $x_{3}=a \in \mathbb{Z}_{3}$, where either $a=\overline{1}$ or $a=-\overline{1}$. On the other hand, $x_{5}=x_{4}+a, x_{6}=x_{4}-a$, so $\overline{0} \in \mathbb{Z}_{3}=\left\{x_{4}, x_{5}, x_{6}\right\}$. The contradiction completes the proof.

The above results show that these three classes of graphs are extremal cases of Theorem 5.1.1 when equality in (5.3) holds. We shall prove that they are the only extremal graphs, mainly in the next section.

Lemma 5.1.8 Let $G$ be a 2-connected graph. Each of the following holds.

(i) If $\Delta(G)=2$, then $\Lambda_{g}(G)=\beta_{2}(G)+1$ if and only if $G \cong C_{m}$ for some integer $m \geq 2$.

(ii) If $\Delta(G) \geq 3$ and if $G$ is not simple, then $\Lambda_{g}(G)=\beta_{2}(G)+1$ if and only if $G=K_{2, s}^{1}$ for some integer $s \geq 3$.

(iii) Let $G$ be a graph with $\operatorname{girth}(G)=k \geq 3$ and let $C^{1}, C^{2}$ be two distinct $k$-circuits in $G$. If $C^{1}$ and $C^{2}$ have at least one common edge, then the intersection of $C^{1}$ and $C^{2}$ must be a path of length at most $k / 2$.

Proof: (i) follows from Lemma 1.3.3 (ii). Suppose that $G$ has parallel edges. By $(5.5), \beta_{2}(G)=$ $\operatorname{girth}(G)=2$. Thus (ii) follows from Lemma 5.1.6. Part (iii) follows from the assumption that $\operatorname{girth}(G)=k$.

Proof of Corollary 5.1.2: $\quad$ By (5.3), $\Lambda_{g}(G) \leq \beta_{2}(G)+1 \leq c(G)+1$. By (5.5), when the equality holds in (5.4), we must have $\operatorname{girth}(G)=\beta_{2}(G)=c(G)$. As $g\left(K_{4}^{1 / k}\right)<c\left(K_{4}^{1 / k}\right)$, Corollary 5.1.2 follows from Theorem 5.1.1.

Proof of Corollary 5.1.3: Let $H_{1}, H_{2}, \ldots, H_{s}$ be the blocks of $G$. If $\Lambda_{g}(G)=c(G)+1$, then by Lemma 1.3.8, some $H_{i}$ has its group connectivity number equal to $c(G)+1$, in which case (4) implies $c\left(H_{i}\right)=c(G)$. Without loss of generality, and by Theorem 5.1.1, we may assume that $\Lambda_{g}\left(H_{i}\right)=c(G)+1$ for $H_{i} \in\left\{H_{1}, \ldots, H_{s^{\prime}}\right\}$ and $\Lambda_{g}\left(H_{i}\right) \leq c(G)$ for $H_{i} \in\left\{H_{s^{\prime}+1}, \ldots, H_{s}\right\}$. Thus Corollary 5.1.3 follows from Corollary 5.1.2. 


\subsection{Characterization of the Extremal Graphs}

By Lemma 5.1.8, it suffices to characterize the extremal graphs for 2-connected simple graphs $G$ with $\operatorname{girth}(G) \geq 3$ and $\Delta(G) \geq 3$. Moreover, the intersection of any two circuits in $G$ has at most $\lfloor k / 2\rfloor$ edges.

Define $C^{2}(k, l)$, where $1 \leq l \leq k / 2$, to be the union of two $k$-circuits whose intersection is a path of length $l$; and $C^{3}\left(k, l_{1}, l_{2}, l_{3}\right)$, where $1 \leq l_{1}, l_{2}, l_{3} \leq k / 2$ and $l_{1}+l_{2}+l_{3} \leq k$, to be the union of three $k$-circuits among which the intersection of any two circuits is a path of length $l_{1}, l_{2}, l_{3}$, respectively. See Figure 5.2 for examples of these graphs.
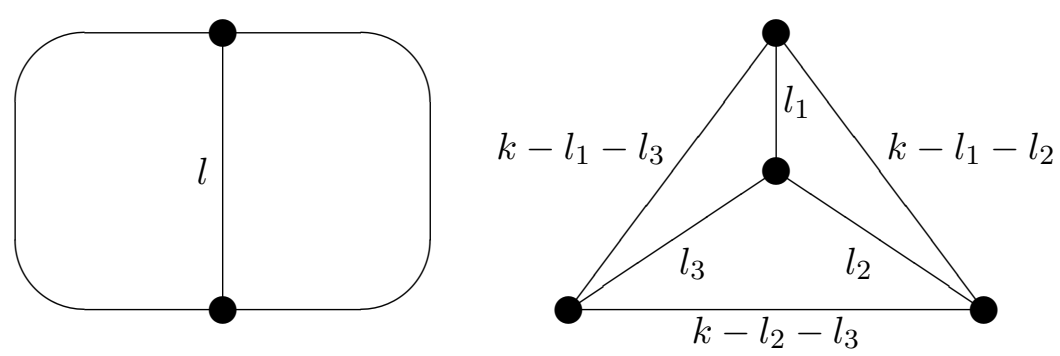

Figure $5.2 C^{2}(k, l)$ and $C^{3}\left(k, l_{1}, l_{2}, l_{3}\right)$.

Lemma 5.2.1 If $l_{1}, l_{2}, l_{3}$ are not identically equal, then $\Lambda_{g}\left(C^{3}\left(k, l_{1}, l_{2}, l_{3}\right)\right) \leq k$.

Proof: Let $A$ be a group of order at least $k$. Without loss of generality, we assume that $l_{1}<l_{3}$. Let $H=C^{3}\left(k, l_{1}, l_{2}, l_{3}\right)$ be annotated and oriented as in Figure 5.3. We shall adopt the same notation as in the proof of Lemma 5.1.7 and denote $P^{\left(v_{i}, v_{j}\right)}$ to be the undirected $\left(v_{i}, v_{j}\right)$-path of which all the internal vertices have degree 2 . Let $\bar{f} \in F(H, A)$. We shall construct an $A$-flow $f$ such that $f(e) \neq \bar{f}(e)$ for any $e$ in $H$. Thus by Theorem 1.3.2, $H$ is $A$-connected for any $A$ with $|A| \geq k$.

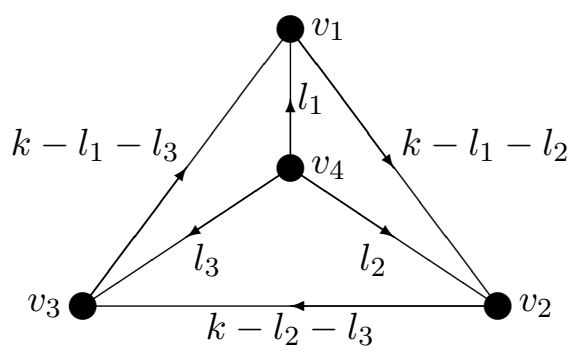


Figure 5.3 Oriented $C^{3}\left(k, l_{1}, l_{2}, l_{3}\right)$.

Denote $\bar{f}\left(P^{\left(v_{1}, v_{4}\right)}\right)=\left\{\bar{a}_{1}, \cdots, \bar{a}_{l_{1}}\right\}, \bar{f}\left(P^{\left(v_{2}, v_{4}\right)}\right)=\left\{\bar{b}_{1}, \cdots, \bar{b}_{l_{2}}\right\}, \bar{f}\left(P^{\left(v_{3}, v_{4}\right)}\right)=\left\{\bar{c}_{1}, \cdots, \bar{c}_{l_{3}}\right\}$, $\bar{f}\left(P^{\left(v_{1}, v_{2}\right)}\right)=\left\{\bar{x}_{1}, \cdots, \bar{x}_{k-l_{1}-l_{2}}\right\}, \bar{f}\left(P^{\left(v_{2}, v_{3}\right)}\right)=\left\{\bar{y}_{1}, \cdots, \bar{y}_{k-l_{2}-l_{3}}\right\}$, and $\bar{f}\left(P^{\left(v_{3}, v_{1}\right)}\right)=\left\{\bar{z}_{1}, \cdots, \bar{z}_{k-l_{1}-l_{3}}\right\}$.

Claim 1 There exist $x, y, z \in A$ satisfying each of the following:

(i) $y \in A-\left\{\bar{y}_{1}, \cdots, \bar{y}_{k-l_{2}-l_{3}}\right\}$;

(ii) $x \in A-\left\{\bar{x}_{1}, \cdots, \bar{x}_{k-l_{1}-l_{2}}, y-\bar{b}_{1}, \cdots, y-\bar{b}_{l_{2}}\right\}$;

(iii) $z \in A-\left\{\bar{z}_{1}, \cdots, \bar{z}_{k-l_{1}-l_{3}}, y+\bar{c}_{1}, \cdots, y+\bar{c}_{l_{3}}, x-\bar{a}_{1}, \cdots, x-\bar{a}_{l_{1}}\right\}$.

Since $|A| \geq k \geq l_{1}+l_{2},+l_{3},\left|A-\left\{\bar{y}_{1}, \cdots, \bar{y}_{k-l_{2}-l_{3}}\right\}\right| \geq k-l_{1} \geq l_{2}+l_{3}$. An element $y$ satisfying (i) has at least $l_{2}+l_{3}$ choices. Since $l_{1}<l_{3}$, we have $l_{2}+l_{3}>l_{1}+l_{2}$. If

$$
\left|\left\{\bar{x}_{1}, \cdots, \bar{x}_{k-l_{1}-l_{2}}\right\}\right|<k-l_{1}-l_{2}
$$

then there exists $y \in A-\left\{\bar{y}_{1}, \cdots, \bar{y}_{k-l_{2}-l_{3}}\right\}$ such that

$$
\left|\left\{\bar{x}_{1}, \cdots, \bar{x}_{k-l_{1}-l_{2}}, y-\bar{b}_{1}, \cdots, y-\bar{b}_{l_{2}}\right\}\right|<k-l_{1}
$$

and so an $x$ satisfying (ii) can also be chosen. If $\left|\left\{\bar{x}_{1}, \cdots, \bar{x}_{k-l_{1}-l_{2}}\right\}\right|=k-l_{1}-l_{2}$ and $y_{1}-$ $\bar{b}_{1}, \cdots, y_{l_{2}+l_{3}}-\bar{b}_{1}$ are $\left(l_{2}+l_{3}\right)$ distinct elements, then $y_{i}-\bar{b}_{1} \in\left\{\bar{x}_{1}, \cdots, \bar{x}_{k-l_{1}-l_{2}}\right\}$ for some $i$, and so (5.7) holds as well. Hence we can find $x$ and $y$ satisfying both (i) and (ii) in either case.

With a similar argument, for a given $y$, either $\left|\left\{\bar{z}_{1}, \cdots, \bar{z}_{k-l_{1}-l_{3}}, y+\bar{c}_{1}, \cdots, y+\bar{c}_{l_{3}}\right\}\right|<k-l_{1}$ or we can choose $x$ such that $x-\bar{a}_{1} \in\left\{\bar{z}_{1}, \cdots, \bar{z}_{k-l_{1}-l_{3}}, y+\bar{c}_{1}, \cdots, y+\bar{c}_{l_{3}}\right\}$. In either case, $\left|\left\{\bar{z}_{1}, \cdots, \bar{z}_{k-l_{1}-l_{3}}, y+\bar{c}_{1}, \cdots, y+\bar{c}_{l_{3}}, x-\bar{a}_{1}, \cdots, x-\bar{a}_{l_{1}}\right\}\right|<k$ and so there must be at least a $z$ satisfying (iii). This proves Claim 1.

By Claim 1, there exist $x, y, z \in A$ satisfying Claim 1 (i)-(iii). Set $a=x-z, b=y-x$, and $c=z-y$. We define $f: E(H) \mapsto A$ such that $f\left(P^{\left(v_{1}, v_{4}\right)}\right)=\{a\}, f\left(P^{\left(v_{2}, v_{4}\right)}\right)=\{b\}$, $f\left(P^{\left(v_{3}, v_{4}\right)}\right)=\{c\}, f\left(P^{\left(v_{1}, v_{2}\right)}\right)=\{x\}, f\left(P^{\left(v_{2}, v_{3}\right)}\right)=\{y\}$, and $f\left(P^{\left(v_{3}, v_{1}\right)}\right)=\{z\}$. Note that $f$ defines an $A$-flow on $H$. Moreover, by Claim 1 (ii) and (iii),

$$
a \notin\left\{\bar{a}_{1}, \cdots, \bar{a}_{l_{1}}\right\}, b \notin\left\{\bar{b}_{1}, \cdots, \bar{b}_{l_{2}}\right\}, c \notin\left\{\bar{c}_{1}, \cdots, \bar{c}_{l_{3}}\right\},
$$

and

$$
x \notin\left\{\bar{x}_{1}, \cdots, \bar{x}_{k-l_{1}-l_{2}}\right\}, y \notin\left\{\bar{y}_{1}, \cdots, \bar{y}_{k-l_{2}-l_{3}}\right\} \text {, and } z \notin\left\{\bar{z}_{1}, \cdots, \bar{z}_{k-l_{1}-l_{3}}\right\} .
$$

Hence $f(e) \neq \bar{f}(e)$ for any $e$ in $H$. This completes the proof. 


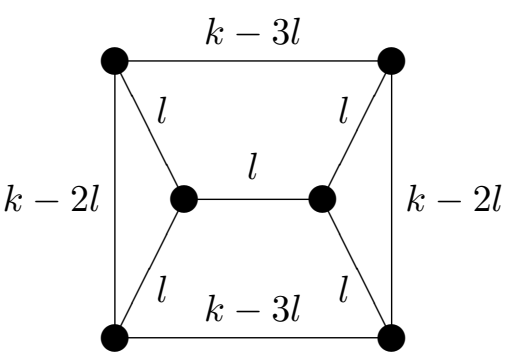

(a)

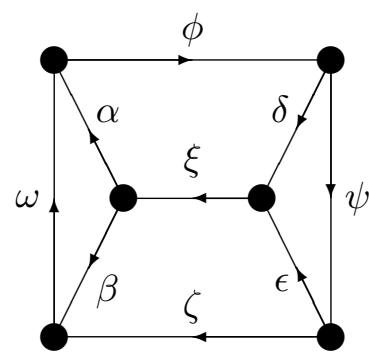

(b)

Figure 5.4

Lemma 5.2.2 Let $K_{3} \times K_{2}$ denote the Cartesian product (see Page 30 of [2]) of the complete graphs $K_{3}$ and $K_{2}$. For integers $k, l$ with $k \geq 3 l>0$, let $H$ denote the subdivided $K_{3} \times K_{2}$ as depicted in Figure 5.4(a), where the integer $i_{e}$ on each edge e of $K_{3} \times K_{2}$ indicates that the edge $e$ is subdivided into a path of $i_{e}$ edges. If $k>3 l$, then $H$ is $A$-connected for any $A$ with $|A| \geq k$.

Proof: Let $A$ be an Abelian group of order at least $k$, and assume that $H$ is oriented as in Figure 5.4(b). Let $\bar{f} \in F(H, A)$. We shall adopt the convention so that the path labelled with $\gamma \in\{\alpha, \beta, \xi, \delta, \epsilon, \phi, \psi, \zeta, \omega\}$ in Figure 5.4(b) is denoted as $P_{\gamma}$, and the values that $\bar{f}$ has assigned on the edges in $P_{\gamma}$ are denoted as $\bar{\gamma}_{1}, \bar{\gamma}_{2}, \cdots$. We will show $\Lambda_{g}(H) \leq k$ by applying the equivalence between Theorem 1.3.2(iii) and Theorem 1.3.2(i) again.

Claim 2 There exist $x, y, z, w \in A$ satisfying each of the following:

(i) $x \in A-\left\{\bar{\phi}_{1}, \cdots, \bar{\phi}_{k-3 l}\right\}$,

(ii) $y \in A-\left\{\bar{\psi}_{1}, \cdots, \bar{\psi}_{k-2 l}, x-\bar{\delta}_{1}, \cdots, x-\bar{\delta}_{l}\right\}$,

(iii) $z \in A-\left\{\bar{\zeta}_{1}, \cdots, \bar{\zeta}_{k-3 l}, y-\bar{\epsilon}_{1}, \cdots, y-\bar{\epsilon}_{l}, x-\bar{\xi}_{1}, \cdots, x-\bar{\xi}_{l}\right\}$,

(iv) $w \in A-\left\{\bar{\omega}_{1}, \cdots, \bar{\omega}_{k-2 l}, x-\bar{\alpha}_{1}, \cdots, x-\bar{\alpha}_{l}, z+\bar{\beta}_{1}, \cdots, z+\bar{\beta}_{l}\right\}$.

The following observations are straightforward.

$$
\forall x \in A,\left|\left\{\bar{\psi}_{1}, \cdots, \bar{\psi}_{k-2 l}, x-\bar{\delta}_{1}, \cdots, x-\bar{\delta}_{l}\right\}\right| \leq k-l<k,
$$

and

$$
\forall x, y \in A,\left|\left\{\bar{\zeta}_{1}, \cdots, \bar{\zeta}_{k-3 l}, y-\bar{\epsilon}_{1}, \cdots, y-\bar{\epsilon}_{l}, x-\bar{\xi}_{1}, \cdots, x-\bar{\xi}_{l}\right\}\right| \leq k-l<k .
$$

Denote $A-\left\{\bar{\phi}_{1}, \cdots, \bar{\phi}_{k-3 l}\right\}=\left\{x_{1}, \cdots, x_{t}\right\}$, where $t \geq 3 l$. If $\left|\left\{\bar{\omega}_{1}, \cdots, \bar{\omega}_{k-2 l}\right\}\right|<k-2 l$, pick any $x \in\left\{x_{1}, \cdots, x_{t}\right\}$. Otherwise $\left|\left\{\bar{\omega}_{1}, \cdots, \bar{\omega}_{k-2 l}\right\}\right|=k-2 l>k-3 l$, and so there exists an $i$ such that $x_{i}-\bar{\alpha}_{1} \in\left\{\bar{\omega}_{1}, \cdots, \bar{\omega}_{k-2 l}\right\}$. Hence we can pick $x=x_{i}$. Thus in either case, there always exists an $x$ so that $\left|\left\{\bar{\omega}_{1}, \cdots, \bar{\omega}_{k-2 l}, x-\bar{\alpha}_{1}, \cdots, x-\bar{\alpha}_{l}\right\}\right|<k-l$. 
After $x$ has been chosen, pick any $y \in A-\left\{\bar{\psi}_{1}, \cdots, \bar{\psi}_{k-2 l}, x-\bar{\delta}_{1}, \cdots, x-\bar{\delta}_{l}\right\}$; and pick any $z \in A-\left\{\bar{\zeta}_{1}, \cdots, \bar{\zeta}_{k-3 l}, y-\bar{\epsilon}_{1}, \cdots, y-\bar{\epsilon}_{l}, x-\bar{\xi}_{1}, \cdots, x-\bar{\xi}_{l}\right\}$. By the choice of $x, \mid\left\{\bar{\omega}_{1}, \cdots, \bar{\omega}_{k-2 l}, x-\right.$ $\left.\bar{\alpha}_{1}, \cdots, x-\bar{\alpha}_{l}, z+\bar{\beta}_{1}, \cdots, z+\bar{\beta}_{l}\right\} \mid<k$. Hence there exists $w \in A-\left\{\bar{\omega}_{1}, \cdots, \bar{\omega}_{k-2 l}, x-\bar{\alpha}_{1}, \cdots, x-\right.$ $\left.\bar{\alpha}_{l}, z+\bar{\beta}_{1}, \cdots, z+\bar{\beta}_{l}\right\}$. This proves Claim 2 .

By Claim 2, there exist $x, y, z, w \in A$ satisfying Claim 2 (i)-(iv). Set $a=x-w, b=w-z$, $c=x-z, d=x-y$, and $e=y-z$. Define $f: E(H) \mapsto A$ in such a way that $f$ takes a constant value $f(\gamma)$ on every edge of $P_{\gamma}$, for all $\gamma \in\{\alpha, \beta, \xi, \delta, \epsilon, \phi, \psi, \zeta, \omega\}$, as follows:

$$
f=\left(\begin{array}{lllllllll}
\alpha & \beta & \xi & \delta & \epsilon & \phi & \psi & \zeta & \omega \\
a & b & c & d & e & x & y & z & w
\end{array}\right) .
$$

For notational convenience, we also view $f$ as a bijection from $\{\alpha, \beta, \xi, \delta, \epsilon, \phi, \psi, \zeta, \omega\}$ onto $\{a, b, c$, $d, e, x, y, z, w\}$.

As a mapping $f: E(H) \mapsto A$ under the indicated orientation in Figure 3.3(b), $f$ defines an $A$-flow on $H$. Moreover, by Claim 2 (i)-(iv), for all $\gamma \in\{\alpha, \beta, \xi, \delta, \epsilon\}, f(\gamma) \notin\left\{\bar{\gamma}_{i}: i=1,2, \ldots, l\right\}$ and $y \notin\left\{\bar{\psi}_{i}: 1 \leq i \leq k-2 l\right\}, w \notin\left\{\bar{\omega}_{i}: 1 \leq i \leq k-2 l\right\}, z \notin\left\{\bar{\zeta}_{i}: 1 \leq i \leq k-3 l\right\}$, and $x \notin\left\{\bar{\phi}_{i}: 1 \leq i \leq k-3 l\right\}$. Hence $f(e) \neq \bar{f}(e)$, for any $e \in E(H)$. This completes the proof.

From now on in this section, we assume that

$$
G \in \mathcal{E}_{k} \text { and } \Delta(G) \geq 3
$$

By (5.6), $\operatorname{girth}(G)=\beta_{2}(G)=k$ and $\Lambda_{g}(G)=\beta_{2}(G)+1$. We shall prove that either $G=K_{2, \Delta(G)}^{1 /(k / 2)}$ and $k$ is even, or $G=K_{4}^{1 /(k / 3)}$ and $k \equiv 0(\bmod 3)$.

Let $v$ be a vertex of degree at least 3 in $G$ and let $e_{1}, e_{2}, e_{3}$ be three edges incident with $v$. By the definition of $\beta_{2}$, there exists a $k$-circuit $C^{i j}$ such that $e_{i}, e_{j} \in C^{i j}$ for any $1 \leq i<j \leq 3$. Let $U$ denote the union of the $C^{i j}$ 's. Then $U$ is either $K_{2,3}^{1 /(k / 2)}$ or $C^{3}\left(k, l_{1}, l_{2}, l_{3}\right)$ for some $l_{1}, l_{2}, l_{3}$.

Lemma 5.2.3 If $G$ satisfies (5.8) and contains $C^{3}(k, l, l, l)$, then $l=k / 3$.

Proof: By the definition of $C^{3}(k, l, l, l)$, we have $1 \leq l \leq k / 3$. We argue by contradiction and assume that $l<k / 3$ and that $G$ contains $C^{3}(k, l, l, l)$ as depicted and annotated in Figure 5.5, where $v_{1}, v_{2}$ are two neighbors of $v$. Let $C^{1}$ and $C^{2}$ denote the two $k$-circuits containing $v$ in this subgraph. Now consider the adjacent edges $v_{1} v, v_{2} v$, and let $C$ be a $k$-circuit in $G$ containing these two edges. Note that $C^{1} \cup C^{2} \cup C$ is a subgraph of $G$ that is not $K_{2,3}^{1 /(k / 2)}$. Since $C^{1}$ and $C^{2}$ intersect in a path of length $l<k / 2$, by Lemma 5.2 .1 it must also be a $C^{3}(k, l, l, l)$. Hence $G$ contains a subgraph $H$ as depicted in Figure 5.4(a). Since $\beta_{2}(H)=k$, it follows by Lemma 5.2.2 that $H$ is $A$-connected for any $A$ with $|A| \geq k$. By the definition of $\beta_{2}(G)$, if $H \neq G$, then any edge $e \in E(H)$ adjacent to an edge in $E(G)-E(H)$ must be in a circuit of length at most $k$, and so by the 2-edge-connectedness of $G$, the closure $c l_{\beta_{2}(G)-1}(H)=G$. It follows by 
Corollary 5.1.4 that $\Lambda_{g}(G) \leq k$, contrary to the assumption that $\Lambda_{g}(G)=\beta_{2}(G)+1$. Hence we must have $k=3 l$.

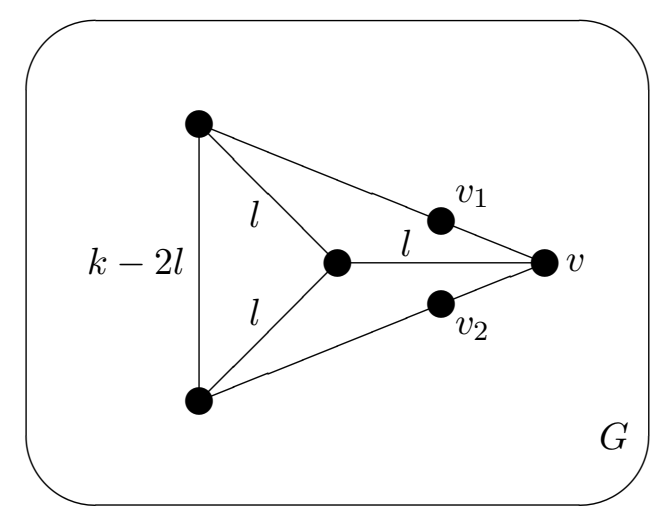

Figure 5.5

Lemma 5.2.4 If $G$ satisfies (5.8) and contains $C^{2}(k, l)$ with $1 \leq l<k / 2$, then $l=k / 3$ and $G$ contains $C^{3}(k, l, l, l)=K_{4}^{1 / l}$.

Proof: Let $C^{1}$ and $C^{2}$ be two $k$-circuits in $G$ that intersect in a path of length $l$. Let $v$ be an endpoint of the intersection path, and let $v^{\prime}$ and $v^{\prime \prime}$ be the two neighbors of $v$, with $v^{\prime} \in C^{1}-C^{2}$ and $v^{\prime \prime} \in C^{2}-C^{1}$. Let $C$ be a $k$-circuit containing $v v^{\prime}$ and $v v^{\prime \prime}$. Since $l<k / 2$ and $C \neq C^{1} \triangle C^{2}$, $C^{1} \cup C^{2} \cup C$ is isomorphic $C^{3}(k, l, m, n)$ for some $m, n$. By Lemma 5.2.1, $l=m=n$, so $G$ contains a $C^{3}(k, l, l, l)$. By Lemma $5.2 .3, l=k / 3$.

Lemma 5.2.5 If $G$ satisfies (5.8), then each of the following holds:

(i) If $k \equiv 0(\bmod 3)$, and if $C^{1}$ and $C^{2}$ are two $k$-circuits in $G$ which intersect in a path of length $k / 3$, then any internal vertex in this path has degree 2 in $G$.

(ii) Suppose that $k$ is even and that any two circuits of $G$ intersect in a path of length either 0 or $k / 2$. If $G$ contains $H=K_{2,3}^{1 /(k / 2)}$ as a subgraph, and $u$ and $v$ are the two degree 3 vertices, then $d_{G}(w)=2$ for all $w \in V(H)-\{u, v\}$.

Proof: (i) Let $P=C^{1} \cap C^{2}$, and let $u$ and $v$ be the endpoints of $P$. If $P$ has an internal vertex $w$ with $d_{G}(w)>2$, then there exists $e, e^{\prime} \in E(G)$ incident with $w$ such that $e \in E\left(C^{1}\right) \cap E\left(C^{2}\right)$ and $e^{\prime} \notin E\left(C^{1}\right) \cap E\left(C^{2}\right)$. By definition of $\beta_{2}, G$ has a $k$-circuit $C$ containing both $e$ and $e^{\prime}$. Since $e \in E\left(C^{1}\right) \cap E\left(C^{2}\right)$, by Lemmas 5.1.8 and 5.2.4, $C$ and $C^{i}$ intersect in a path of length at least $k / 3$ for $i=1,2$. However, since $w$ is an internal vertex of $P$, this is not possible. The contradiction proves (i).

(ii) Denote the three paths in $H$ by $P^{i}, 1 \leq i \leq 3$, respectively. Let $w$ be an internal vertex of $P^{i}$. Arguing similarly as in (i), we conclude that $d_{G}(w)=2$. 
Corollary 5.2.6 If $G$ satisfies (5.8) and contains a subgraph $H=K_{n}^{1 /(k / 3)}$ for some $n \geq 4$ and $k \equiv 0(\bmod 3)$, then for any $v \in V(H)$ with $d_{H}(v)=2, d_{G}(v)=2$.

Proof: This follows from Lemma 5.2.5(i).

Lemma 5.2.7 If $l$ is a positive integer, then $E_{g}\left(K_{5}^{1 /(l)}\right) \leq 3 l$.

The proof of Lemma 5.2.7 use Proposition 5.2.8 below. Let $H$ be a graph and let $v \in V(H)$ with $d=d(v) \geq 4$. Denote $N(v)=\left\{v_{1}, \cdots, v_{d}\right\}$ and denote $e_{i}=v v_{i}$. Define $H_{i j}=H-\left\{e_{i}, e_{j}\right\}+$ $v_{i} v_{j}$.

Proposition 5.2.8 (Lemma 3.1 (i) of [21]) If $H_{i j} \in\langle A\rangle$ for some $i \neq j$, then $H \in\langle A\rangle$.

Proof of Lemma 5.2.7: Let $A$ be an Abelian group of order at least $3 l$. Let $H=K_{5}^{1 / l}$ and let $v$ be a vertex of degree 4 in $H$. Denote the 4 neighbors of $v$ by $v_{1}, v_{2}, v_{3}, v_{4}$. Let $H_{12}=$ $H-\left\{v v_{1}, v v_{2}\right\}+v_{1} v_{2}$. Let $C$ be the $3 l$-circuit containing $v v_{1}, v v_{2}$ and $C^{\prime}=C-\left\{v v_{1}, v v_{2}\right\}+v_{1} v_{2}$. Now $C^{\prime}$ has length $3 l-1$ and thus is $A$-connected. Also $c l_{2 l}\left(C^{\prime}\right)=H_{12}$ and thus $H_{12} \in\langle A\rangle$. By Proposition 5.2.8, $H \in\langle A\rangle$.

Lemma 5.2.9 If $G$ satisfies (5.8) and contains a $K_{4}^{1 /(k / 3)}$, then $G=K_{4}^{1 /(k / 3)}$.

Proof: Let $H=K_{4}^{1 /(k / 3)}$ be a subgraph of $G$. Denote the four vertices of degree 3 in $H$ by $v_{1}, v_{2}, v_{3}, v_{4}$, and refer to all other vertices in $H$ as internal vertices of $H$. For $1 \leq i<j \leq 4$, let $P^{i j}$ denote the $\left(v_{i}, v_{j}\right)$-path in $H$ of length $k / 3$, and for $1 \leq i<j<s \leq 4$ let $C^{i j s}$ denote the $k$-circuit in $H$ containing $v_{i}, v_{j}, v_{s}$.

Assume that $G \neq H$. By Corollary 5.2.6, every internal vertex of $H$ has degree 2 in $G$. Since $G \neq H$ and $\kappa^{\prime}(G) \geq 2$, we may assume that $v_{4}$ is incident with an edge $e \notin E(H)$. Let $e_{i}$ be the edge incident with $v_{4}$ in $P^{i 4}$ for $1 \leq i \leq 3$. Now $e$ is adjacent with $e_{i}$, for $1 \leq i \leq 3$. Let $C^{i}$ be a $k$-circuit in $G$ containing $e, e_{i}$.

We claim that $C^{1}$ must intersect $C^{124}$ in a path of $k / 3$. Otherwise, by Lemma $5.2 .4, C^{1}$ and $C^{124}$ intersect in a path of length $k / 2$. This marks some internal vertex in $P^{12}$ incident with an edge that is not in $H$. This leads to a contradiction. Hence $C^{1}$ intersects $C^{124}$ exactly in $P^{14}$. Similarly, $C^{1} \cap C^{134}=P^{14}$, and so $C^{1} \cap H=P^{14}$. With similar arguments, $C^{2} \cap H=P^{24}$ and $C^{3} \cap H=P^{34}$.

Note that $C^{1} \neq C^{2}$ and $C^{1} \cap C^{2}=e$. Apply Lemma 5.2.1 to $C^{1} \cup C^{2} \cup C^{124}$ to see that $C^{1}$ and $C^{2}$ must intersect in a path of $k / 3$ containing $e$. Let $u$ be the vertex in the path of $C^{1}-P^{14}$ such that the two paths from $v_{1}$ to $u$ and from $v_{4}$ to $u$ have the same length in $C^{1}$. Let $P$ denote the path in $C^{1}$ from $u$ to $v_{4}$ containing $e$, so $C^{1} \cap C^{2}=P$. Similarly $C^{1} \cap C^{3}=P=C^{2} \cap C^{3}$.

Let $L=H \cup C^{1} \cup C^{2} \cup C^{3}$. It follows by Lemma 5.2.7 that $L=K_{5}^{1 /(k / 3)}$ and that $\Lambda_{g}(L) \leq$ $k$. By repeating applications of Lemma 1.3.3 (ii), $\Lambda_{g}(G / L) \leq k$. By Proposition 1.3.1(C3), $\Lambda_{g}(G) \leq k$, contrary to (5.8). This contradiction proves that $G=H$. 
Lemma 5.2.10 Suppose $G$ satisfies (5.8). If any two circuits of $G$ intersect in a path of length either 0 or $k / 2$, then $G=K_{2, \Delta(G)}^{1 /(k / 2)}$.

Proof: Let $v \in V(G)$ and $d(v)=\Delta(G)$. Denote the edges incident with $v$ by $e_{1}, \ldots, e_{\Delta(G)}$. Let $C^{12}$ be a $k$-circuit containing $e_{1}$ and $e_{2}$, and denote the vertex in $C^{12}$ by $u$ such that either of the two $(u, v)$-paths in $C^{12}$ has the length $k / 2$. Let $P^{1}$ denote the $(u, v)$-path in $C^{12}$ containing $e_{1}$. Let $C^{1 m}$ be the $k$-circuit containing $e_{1}$ and $e_{m}$, where $m \geq 3$. By assumption, $\left|C^{12} \cap C^{1 m}\right|=k / 2$. Hence $C^{12} \cap C^{1 m}=P^{1}$. Let $H=C^{12} \cup \cdots \cup C^{\Delta(G)}$. Note that $H=K_{2, \Delta(G)}^{1 /(k / 2)}$ with $u$ and $v$ as the two common ends of all the paths. By Lemma 5.2.5(ii), the internal vertices of the paths have degree 2 in $G$. Moreover, $d_{H}(u)=d_{H}(v)=d_{G}(v)=\Delta(G) \geq d_{G}(u)$. Hence $G=H$ as $\kappa^{\prime}(G) \geq 2$.

Proof of Theorem 5.1.1: By (5.5), $\Lambda_{g}(G) \leq \beta_{2}(G)+1$. By Lemmas 1.3.3(ii), 5.1.6 and 5.1.7, for $G \in\left\{C_{k}: k \geq 2\right\} \cup\left\{K_{2, t}^{1 / m}: m \geq 1, t \geq 3\right\} \cup\left\{K_{4}^{1 / k}: k \geq 1\right\}$, we have $\Lambda_{g}(G)=\beta_{2}(G)+1$.

Conversely, suppose that $\Lambda_{g}(G)=\beta_{2}(G)+1$. If $\Delta(G)=2$, then Theorem 5.1.1 follows from Lemma 5.1.8. Assume that $\Delta(G) \geq 3$. By Lemmas 5.2.3, 5.2.4, 5.2.9 and 5.2.10, either $k$ is even and $G=K_{2, \Delta(G)}^{1 /(k / 2)}$, or $k \equiv 0(\bmod 3)$ and $G=K_{4}^{1 /(k / 3)}$. This completes the proof of Theorem 5.1.1.

\subsection{Applications}

We have seen that Theorem 5.1.1 can be applied to obtain Corollaries 5.1.2 and 5.1.3. In this section, we shall present additional evidence that Theorem 5.1.1 can be applied to study the group connectivity of certain families of graphs. For subgraphs $H_{1}$ and $H_{2}$ of a graph $G$, define

$$
H_{1} \triangle H_{2}=H_{1} \cup H_{2}-E\left(H_{1}\right) \cap E\left(H_{2}\right) .
$$

Lemma 5.3.1 If a graph $G$ has $\Lambda_{g}(G) \geq m+1$ with $|V(G)|+|E(G)|$ minimized, then $G$ contains no nontrivial subgraph $H$ such that $\Lambda_{g}(H) \leq m$.

Proof: If $G$ has a nontrivial subgraph $H$ with $\Lambda_{g}(H) \leq m$, then $|V(G / H)|+|E(G / H)|<$ $|V(G)|+|E(G)|$, we have $\Lambda_{g}(G / H) \leq m$. Since $\Lambda_{g}(H) \leq m$, by Proposition 1.3.1(C3), $\Lambda_{g}(G) \leq$ $m$, contrary to the assumption of the lemma.

Corollary 5.3.2 (Theorem 3.1, [29]) If $G$ is a 2-edge-connected loopless graph with diameter at most 2, then $\Lambda_{g}(G) \leq 6$, where equality holds if and only if $G$ is a 5-circuit.

Proof: By contradiction, assume that $G$ is a counterexample with $|V(G)|+|E(G)|$ minimized. The diameter of $G$ is at most 2 , but $\Lambda_{g}(G) \geq 7$. By the definition of $\beta_{2}(G), G$ has a 2-path $P_{2}$ 
with $\beta_{2}\left(P_{2}\right)=\beta_{2}(G)$, and hence $G$ has a circuit $C$ containing $P_{2}$ with $|E(C)|=\beta_{2}\left(P_{2}\right)$. Let $V\left(P_{2}\right)=\left\{v_{1}, v_{2}, v_{3}\right\}$ and $V(C)=\left\{v_{1}, v_{2}, \ldots, v_{m}\right\}$. By Theorem 5.1.1, $m \geq 6$.

Since the diameter of the graph $G$ is at most $2, G$ has a $\left(v_{1}, v_{4}\right)$-path $P^{\prime}$ with $\left|E\left(P^{\prime}\right)\right| \leq 2$. Assume $P^{\prime}=v_{1} v^{\prime} v_{4}$. Since $m \geq 6, P^{\prime}$ is not a path on $C$. Let $P$ denote a $\left(v_{1}, v_{4}\right)$-path on $C$ with $|E(P)|=3$. Since $P^{\prime}$ and $P$ are both $\left(v_{1}, v_{4}\right)$-paths, $P^{\prime} \Delta P$ contains a circuit $C^{\prime}$ whose length is at most $\left|E\left(P^{\prime}\right)\right|+|E(P)|=5$. By Lemma 1.3.3 (ii), $G$ has a nontrivial subgraph $C^{\prime}$ with $\Lambda_{g}\left(C^{\prime}\right) \leq 6$, contrary to Lemma 5.3.1 with $m=6$.

A argument similar to the proof above can also be employed to prove the following.

Corollary 5.3.3 If $G$ is a 2-edge-connected loopless graph with diameter at most $m$, where $m \geq 3$, then $\Lambda_{g}(G) \leq 2 m+2$. 


\section{Bibliography}

[1] L. Beineke, Derived graphs and digraphs, Beiträge zur Graphentheorie, Teubner, Leipzig, 1968.

[2] J. A. Bondy and U. S. R.Murty, Graph theory with applications. American Elsevier, New York, 1976.

[3] P. A. Catlin, Supereulerian graph, collapsible graphs and 4-cycles. Congressus Numerantium, 56 (1987) 223-246.

[4] P. A. Catlin, A reduction method to find spanning Eulerian subgraphs, J. Graph Theory $12(1988)$ 29-44.

[5] J. J. Chen, E. Eschen and H. J. Lai, Group connectivity of certain graphs, Ars Combinatoria, 89 (2008) 141-158.

[6] Z. H. Chen, H.-J. Lai and H. Y. Lai, nowhere-zero flows in line graphs, Discrete Mathematics, 230 (2001), 133-141.

[7] Z. H. Chen, H.-J. Lai, X. Lei and X. K. Zhang, Group coloring and group connectivity of graphs, Congressus Numerantium, 134 (1998), 123-130.

[8] M. DeVos, R. Xu, and G.-Y. Yu, Nowhere-zero $\mathbb{Z}_{3}$-flows through $\mathbb{Z}_{3}$-connectivity. Discrete Math. 306 (2006) 26-30.

[9] G. Fan, H.-J. Lai, R. Xu, C.-Q. Zhang and C. Zhou, Nowhere-zero 3-flows in triangularly connected graphs. Journal of Combinatorial Theory, Series B 98 (2008) 1325-1336.

[10] R. J. Gould, Advance on the Hamiltonian Problem - A Survey, Graphs and Combinatorics, 19 (2003), 7-52

[11] F. Harary, C. St. J. A. Nash-Williams, On eulerian an hamilton graphs and line graphs, Canad. Math. Bull. 8 (1965) 701-709.

[12] T. Hungerford, Algebra. Springer-Verlag, New York, (1974). 
[13] W.Imrich and R. Škrekovski, A Theorem on Integer Flows on Cartesian Products of Graphs, J. Graph Theory, 43 (2003), 93-98.

[14] F. Jaeger, Flows and generalized coloring theorems in graphs. J. Combinatorial Theory, Ser. B, 26 (1979), 205-216.

[15] F. Jaeger, Nowhere-zero flow problems, in "Selected Topics in Graph Theory" (L. Beineke and R. Wilson, Eds), Vol. 3. pp91-95 Academic Press, London/New York, 1988.

[16] F. Jaeger, N. Linial, C. Payan and M. Tarsi, Group connectivity of graphs - a nonhomogeneous analogue of nowhere-zero flow properties, J. Combinatorial Theory, Ser. B 56 (1992) 165-182.

[17] M. Kochol, An equivalent version of the 3-flow conjecture, J. Combinatorial Theory, Ser. B, 83 (2001) 258-261.

[18] H.-J. Lai, Graph whose edges are in small cycles, Discrete Mathematics, 94 (1991) 11-22.

[19] H.-J. Lai and C. Q. Zhang, Nowhere-zero 3-flows of highly connected graphs, Discrete Math, 110 (1992) 179-183.

[20] H.-J. Lai, Extending a partial nowhere-zero 4-flow, Jhon Wiley Sons, Inc. J Graph Theory 30: $277-288,1999$.

[21] H.-J. Lai, Group connectivity in 3-edge-connected chordal graph, Graphs and Combinatorics, 16, (2000), 165-176.

[22] H.-J. Lai, R. Xu and J. Zhou, On Group connectivity of graphs, Graphs and Combinatorics (2008) 24: $1-9$

[23] H.-J. Lai, Nowhere-zero 3-flows in locally connected graphs, J. Graph Theory, 42 (2003), 211-219.

[24] H. Li and H.-J. Lai, Group Colorability of Multigraphs, Discrete Math., accepted.

[25] Hong-Jian Lai, Xiangwen Li, Yehong Shao and Mingquan Zhan, Group Connectivity and Group Colorings of Graphs - A Survey. Acta Mathematica Sinica-English Series. 3 (2011), 405-434.

[26] H.-J. Lai, Y. H. Shao, H. Wu and J. Zhou, On $\bmod (2 p+1)$-orientations of graphs, J. of Combinatorial Theory, Series B., 99 (2009), 399-406

[27] H.-J. Lai, L. Miao and Y. H. Shao, Every line graph of a 4-edge-connected graph is $\mathbb{Z}_{3^{-}}$ connected. European Journal od Combinatorics, 30 (2009) 595-601. 
[28] H.-J. Lai, R. Xu and J. Zhou, On group connectivity of graphs, Graphs and Combinatorics, $24(2008) 1-9$.

[29] H.-J. Lai and X. J. Yao, Group connectivity of graphs with diameter at most 2, European J. Combin., 27 (2006) 436-443.

[30] H.-J. Lai and X. Zhang, Group colorability of graphs. Ars Combinatoria, 62 (2002), 299-317.

[31] N. Robertson, unpublished notes, see Page 74 of " Graph Theory" by F. Harary, AddisonWesley, Reading Massachusetts, 1969

[32] Z. Ryjáček, On a Closure Concept in Claw-Free Graphs, Journal of Combinatorial Theory, Series B 70 (1997) 217-224.

[33] P. D. Seymour, Nowhere-zero 6-flows. J. Combin. Theory Ser. B 30 (1981) P. 130-135.

[34] J. Shi and C. Q. Zhang, Nowhere-zero 3-flows in products of graphs, J. Graph Theory, (2005), 79-89.

[35] B. Sudakov, nowhere-zero flows in random graphs, J. Combinatorial Theory, Ser. B, 81 (2001) 209-223.

[36] W. T. Tutte, A contribution on the theory of chromatic polynomial, Canad. J. Math., 6 (1954), 80-91.

[37] W. T. Tutte, on the algebraic theory of graph colourings, J. Combin. Theory, 1(1966) p. 15-50.

[38] R. Xu and C. Q. Zhang, Nowhere-zero 3-flows in squares of graphs. Electronic Journal of Combinatorics 10, R5 (2003).

[39] C. Q. Zhang, Integer flows and cycle covers of graphs, New York: Marcel Dekker, 1997.

[40] Z. Zhang, Y. Zheng and A. Mamut, Nowhere-Zero flows in tensor product of graphs, J. Graph Theory, 54 (2007) 284-292. 